H. Umemura

Nagoya Math. J.

Vol. 119 (1990), 1-80

\title{
BIRATIONAL AUTOMORPHISM GROUPS AND DIFFERENTIAL EQUATIONS
}

\author{
HIROSHI UMEMURA
}

Painlevé studied the differential equations $y^{\prime \prime}=R\left(y^{\prime}, y, x\right)$ without moving critical point, where $R$ is a rational function of $y^{\prime}, y, x$. Most of them are integrated by the so far known functions. There are 6 equations called Painlevé's equations which seem to be irreducible or seem to define new transcendental functions. The simplest one among them is $y^{\prime \prime}=6 y^{2}$ $+x$. Painlevé declared on Comptes Rendus in 1902-03 that $y^{\prime \prime}=6 y^{2}+x$ is irreducible. It seems that R. Liouville pointed out an error in his argument. In fact there are discussions on this subject between Painlevé and Liouville on Comptes Rendus in 1902-03. In 1915 J. Drach published a new proof of the irreducibility of the differential equation $y^{\prime \prime}=6 y^{2}+x$. The both proofs depend on the differential Galois theory developed by Drach. But the differential Galois theory of Drach contains errors and gaps and it is not easy to understand their proofs. One of our contemporaries writes in his book: the differential equation $y^{\prime \prime}=6 y^{2}+x$ seems to be irreducible dans un sens que on ne peut pas songer à préciser. This opinion illustrates well the general attitude of the nowadays mathematicians toward the irreducibility of the differential equation $y^{\prime \prime}=6 y^{2}+x$. Therefore the irreducibility of the differential equation $y^{\prime \prime}=6 y^{2}+x$ remains to be proved. We consider that to give a rigorous proof of the irreducibility of the differential equation $y^{\prime \prime}=6 y^{2}+x$ is one of the most important problem in the theory of differential equations.

In this paper we begin by clarifying the works of Painlevé $[\mathrm{P}]$ since they are not clear and it is indispensable to make them rigorous to prove the irreducibility. In part I we give another proof of the following theorem due to Painlevé: any analytic subgroup $G$ of the birational automorphism group Bir $X$ of an algebraic variety $X$ is contained in an algebraic subgroup of the group Bir $X$ (Corollary (1.33) in Part I). We

\footnotetext{
Received November 17, 1989.
} 
proved this in [U2] by constructing a projective model $\bar{X}$ of $X$ on which the group $G$ operates regularly. Our proof in this paper is more direct. In $\S 2$ of Part II, we characterize the permissible operations, so far known functions or the classical functions. We show that the permissible operations discovered by experience is quite uniform and related with algebraic groups (Theorem (2.19)). In $\S 3$ and $\S 4$ of Part II, we prove the following solvability theorem more or less due to Painlevé. Let $F\left(y^{(n)}, y^{(n-1)}, \cdots\right.$, $y, x)=0$ be an algebraic differential equation such that the general solution $y$ depends rationally on the initial conditions. Then the general solution $y$ can be constructed from the coefficients of $F$ by the permissible operations.

We treat differential equations but we use the language of the algebraic geometry particularly that of E.G.A. which is very effective. For this reason we tried to explain our tools for the non-algebraists.

This research was done when the author stayed at Strasbourg University in 1984/85. He wants to express his hearty thanks to Prof. R. Gérard. Without his constant interest in author's work and encouragement, this work would not certainly have been done.

Added in October 1989. We expected for long time that this paper was to be published in the Proceeding of the Franco-Japanese conference on Differential Equations held at Strasbourg 1985. But recently it comes out that troubles with publishers make the publication of the Proceeding impossible. This paper is the first attempt of understanding systematically Painlevé's Leçons de Stockholm [P], using the language of algebraic geormetry but we find it in a strange situation. In fact, starting from this paper, the following three papers improved parts of this paper:

Nishioka, K.: General solutions depending rationally on arbitrary constants. Nagoya Math. J., 113, 1-6 (1989);

- Differential algebraic function fields depending rationally on arbitrary constants. Nagoya Math. J., 113, 173-179 (1989);

Umemura, H.: Second proof of the irreducibility of the first differential equation of Painlevé, Nagoya Math. J., 117, 125-171 (1990).

We also notice that the very important paper of Nishioka

A note on the transcendency of Painleve's first transcendent, Nagoya Math. J., 109, 63-67 (1988)

is written in the same spirit as this paper. On this subject we quote our 
paper;

Umemura, H.: On the irreducibility of the first differential equation, Algebraic Geometry and Commutative Algebra in Honor of Masayoshi NAGATA, 771-789, Tokyo: Kinokuniya 1987.

\section{Part I. Birational automorphism groups}

\section{$\S 1$. Analytic subgroups and algebraic subgroups of the birational automorphism group of an algebraic variety}

The aim of this section is to give a direct proof to the assertion of Painleve: any analytic subgroup of the birational automorphism group Bir $X$ of an algebraic variety $X$ is contained in an algebraic subgroup of Bir $X$. In fact, this was proved in our preceding paper [U2]. The idea of the proof was as follows. Let $G$ be an analytic subgroup of $\operatorname{Bir} X$. Then we can find a projective model $\tilde{X}$ of $X$ on which $G$ operates regularly. Thus the group $G$ is contained in the biregular automorphism group Aut $\tilde{X}$, which is algebraic by G.A.G.A. [G1] and by [G2].

Painlevé states this in his Stockholm Lessons, Quinzième leçons p. 260. His idea of the proof given there may be interesting but there are several subtle problems if we want to complete his proof in a rigorous way.

Therefore the following 2 points are particularly important.

(1) To give the strict definitions of several basic notions; for example algebraic subgroups or an analytic subgroups in a birational automorphism group.

(2) The resulting theorem according to the adopted definitions should be useful as Painlevé applied his assertion to the integration of non-linear differential equations.

For these reasons as in our preceding papers, we have to begin with some unpleasant definitions. We apply our theorem to the integration of non-linear differential equations in Part II, $\S 4$. We have considerably simplified this part by our theorems (3.18) and (4.3) in Part II.

For the convenience of the reader, we recall as briefly as possible the definitions and results in our papers.

In this section every algebraic variety, scheme and morphisms between them are defined over the field $\mathbb{C}$ of complex numbers and by a general point of $X$, we mean any point of a certain non-empty Zariski open set of $X$. 
Let $X$ be a smooth algebraic variety defined over $\mathbb{C}$. In the study of the algebraic structure of the birational automorphism $\operatorname{Bir} X$, the most natural way is to consider $\operatorname{Bir} X$ as a group functor on the category of schemes over $\mathbb{C}([D])$. For an algebraic variety $T$ over $\mathbb{C}$ the value of the group functor $\operatorname{Bir} X$ (denoted Autbirat in [U1]) at $T$ is given by Bir $X(T)=$ birational automorphism $f: X \times T \cdots X \times T \mid f$ commutes with the projection $p_{2}: X \times T \rightarrow T$, i.e. $p_{2} \circ f=p_{2}$ and $f$ defines a biregular isomorphism between open sets $U, V \subset X \times T$ with $U \cap X \times t \neq \varnothing, V \cap X \times$ $t \neq \varnothing$ for any point $t \in T\} / \sim$. (An open subset $W$ of $X \times T$ such that $W \cap X \times t=\varnothing$ for any point $t \in T$ is called $T$-open set.) Two birational automorphism of $X \times T$ are identified if they coinside on an dense $T$-open set. Intuitively the value $\operatorname{Bir} X(T)$ is the set of birational automorphisms of $X$ parametrized by $T$. If $T$ is a $\mathbb{C}$-scheme, then in the above definition of Bir $X(T)$, the birational automorphism should be replaced by its generalization pseudo-automorphism in E.G.A. Chap. IV

Let $Y$ be a $\mathbb{C}$-scheme. A morphism $Y \rightarrow \operatorname{Bir} X$ is a morphism of functors $h_{Y} \rightarrow \operatorname{Bir} X$ on the category of $\mathbb{C}$-schemes.

Let us recall the definition of an algebraic group germ.

Definition (1.1). An algebraic group germ is a system $(G, 1, \theta, m)$ satisfying the following conditions:

(i ) $G$ is an (irreducible) algebraic variety;

(ii) 1 is a point of $G$;

(iii) $\theta$ is a rational map of $G$ to $G$ which is regular in a neighbourhood of 1 ;

(iv) $m$ is a rational map of $G \times G$ to $G$ regular on an open set $\varnothing \neq \Omega$ of $G \times G$;

(v) for any $g \in G$, we have $(1, g) \in \Omega,(g, 1) \in \Omega, m(1, g)=m(g, 1)=g$;

(vi) for any $g \in G$ such that $\theta(g)$ is defined, we have $(\theta(g), g), g) \in \Omega$ and $m(g, \theta(g))=m(\theta(g), g)=1$;

(vii) for any $g, h, k \in G$ satisfying $(g, h) \in \Omega,(h, k) \in \Omega,(m(g, h), k) \in \Omega$, $(g, m(h, k)) \in \Omega$, we have $m(m(g, h), h)=m(g, m(h, k))$.

We denote $m(g, h)$ by $g h$ or by $g \cdot h$ and $\theta(g)$ by $g^{-1}$. We often denote $(G, 1, \theta, m)$ simply by $G$.

See $\S 2[\mathrm{U} 1]$.

Definition (1.2). Let $G$ be an algebraic group germ and $X$ an algebraic variety. An algebraic pseudo-operation $(G, \psi, X)$ is a rational map 
$\varphi: G \times X \mapsto X$ satisfying the following conditions:

(i) the rational map $G \times X \cdot \rightarrow G \times X(g, x) \mapsto(g, \psi(g, x))$ is dominant;

(ii) the following diagram is commutative;

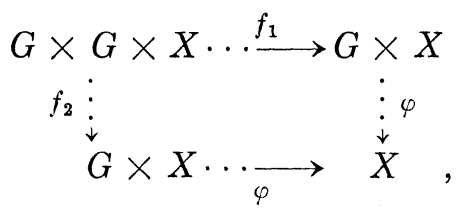

where $f_{1}\left(\left(g_{1}, g_{2}, x\right)\right)=\left(g_{1} g_{2}, x\right), f_{2}\left(\left(g_{1}, g_{2}, x\right)\right)=\left(g_{1}, \varphi\left(g_{2}, x\right)\right)$ by definition for general $g_{1}, g_{2} \in G, x \in X$.

Definition (1.3). Let $\left(G_{i}, \psi_{i}, X_{i}\right)$ be an algebraic pseudo-operation $(i=1,2)$. A morphism $(\varphi, f):\left(G_{1}, X_{1}\right) \rightarrow\left(G_{2}, X_{2}\right)$ of algebraic pseudo-operations consists of a morphisms $\varphi: G_{1} \mapsto G_{2}$ of algebraic group germs (see [U1]) and a dominant rational map $f: X_{1} \cdots X_{2}$ such that the diagram below is commutative:

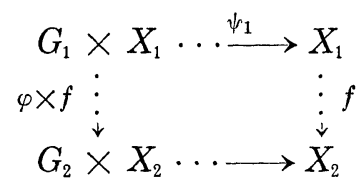

We say that $\left(G_{1}, X_{1}\right)$ and $\left(G_{2}, X_{2}\right)$ are isomorphic if there exist morphisms $\left(\varphi_{1}, f_{1}\right):\left(G_{1}, X_{1}\right) \rightarrow\left(G_{2}, X_{2}\right)$ and $\left(\varphi_{2}, f_{2}\right):\left(G_{2}, X_{2}\right) \rightarrow\left(G_{1}, X_{1}\right)$ such that $\varphi_{1} \circ \varphi_{2}$ and $\varphi_{2} \circ \varphi_{1}$ are identity around 1 and $f_{1} \circ f_{2}$ and $f_{2} \circ f_{1}$ are equivalent to the identities $1_{X_{2}}, 1_{X_{1}}$.

Definition (1.4). An algebraic subgroup of Bir $X$ is a group subfunctor of $\operatorname{Bir} X$ representable by an algebraic group.

The following proposition is very useful ([D]).

Proposition (1.5). Let $\varphi: G \rightarrow \operatorname{Bir} X$ be a morphism of group functors of an algebraic group $G$ to $\operatorname{Bir} X$. Then the $\operatorname{Ker} \varphi$ is representable by a closed subgroup of $G$. In particular there exists an algebraic subgroup $G^{\prime} \hookrightarrow \operatorname{Bir} X$ such that $\varphi$ factors through $G^{\prime} \hookrightarrow \operatorname{Bir} X:$

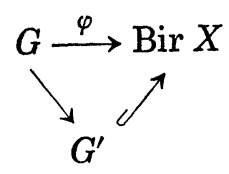

Proposition (1.6). For an algebraic group $G$ and smooth algebraic variety $X$, there is a 1-1 correspondence between the following: 
(i) the set of the morphism of group functors $\operatorname{Hom}_{g r}(G, \operatorname{Bir} X)$ and

(ii) the set of algebraic pseudo-operations $(G, X)$.

Here in the set of (ii) we have to identify isomorphic algebraic pseudooperations.

See Demazure [D] and [U1].

It is convenient to slightly generalize this result. To this end we need.

DeFinition (1.7). Let $G$ be an algebraic group germ. A morphism $\varphi$ : $G \rightarrow \operatorname{Bir} X$ of algebraic group germ functors on the category of $\mathbb{C}$-schemes is a morphism $\varphi: h_{U}=U \rightarrow \operatorname{Bir} X$ of functors of an open neighborhood $U$ of $1 \in G$ to $\operatorname{Bir} X$ such that the diagram

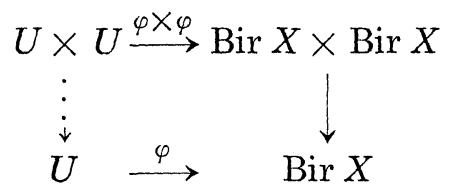

is commutative, where the vertical arrows are the composition laws (namely, the diagram is commutative on a non-empty open set of $U \times U$ ). We identify two morphisms when they coincide on a neighbourhood of $1 \in U$. The set of morphisms of algebraic group germ functors of $G$ modulo above identification to $\operatorname{Bir} X$ is denoted $\operatorname{Hom}_{g g}(G, \operatorname{Bir} X)$.

The argument of the proof of Proposition (1.5) gives us

Lemma (1.8). Let $G$ be an algebraic group germ. There is a 1-1 correspondence between the following:

(i) the set $\operatorname{Hom}_{g g}(G, \operatorname{Bir} X)$ of the morphisms of algebraic group germ functors;

(ii) the set of algebraic pseudo-operations $(G, X)$.

By the same sprit as in the proof of Proposition (1.6), we can show

Lemma (1.9). Let $X$ be a smooth algebraic variety and $Y$ an algebraic variety. Then there is a 1-1 correspondence between the following:

(i) the set $\operatorname{Hom}(Y, \operatorname{Bir} X)$ of the morphisms of functors;

(ii) $\{f: \mid f: Y \times X \cdots Y \times X$ birational inducing a isomorphism of Zariski open sets $U, V$ such that $y \times X \cap U, y \times X \cap V \neq \varnothing$ for any $y \in Y$, $f$ commutes with the projection $Y \times X \stackrel{p_{1}}{\rightarrow} Y ; Y \times X \stackrel{f}{\rightarrow} Y Y \times X$ is commuta${ }^{p_{1}}{ }_{Y}$

tive\}/ , where we identify two birational automorphisms if they coincide 
on a dense $Y$-open set of $Y \times X$.

We shall call an element of the set in (ii) $Y$-birational automorphism of $Y \times X$.

Remark (1.10). Lemma (1.9) is generalized to the case where $Y$ is a scheme. In this case, we take in (ii) $Y$-pseudo-automorphisms of $Y \times X$ (cf. [D] and E.G.A. IV).

Remark (1.11). In our paper ([U1], Definition 2.6), we adopted another definition of algebraic pseudo-operation. But they are equivalent. In fact, let $G$ be an algebraic group germ, $X$ an algebraic variety and $\varphi: G \times X$ .$\rightarrow X$ be a rational map. Then $\varphi$ is an algebraic pseudo-operation if and only if it satisfies the following conditions:

(a) for a general point $x \in X, \varphi$ is regular at $(1, x)$ and $\varphi(1, x)=x$ (in particular $\varphi$ is dominant);

(b) the diagram

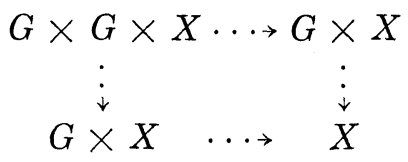

in Definition (1.2) is commutative.

Let us prove this. Assume that $\varphi$ is an algebraic pseudo-operation. As $G \times X \rightarrow G \times X,(g, x) \mapsto(g, g x)$ is dominant, $G \times X \rightarrow G \times X,(g, x) \mapsto$ $\left(g^{-1}, g x\right)$ is dominant. Therefore for general $g \in G$ and $x \in X, \varphi$ is regular at $\left(g^{-1}, g x\right)$ and we can define $g^{-1}(g x)$. Consequently a rational map $G \times$ $X \rightarrow G \times X,(g, X) \mapsto\left(g^{-1}, g^{-1}(g x)\right)$ is dominant and thus $G \times X \rightarrow G \times X$, $(g, x) \rightarrow\left(g, g^{-1}(g x)\right)$ is dominant. Therefore we can define $g\left(g^{-1}(g x)\right)$. On the other hand for general $h, k, j \in G$ and $x \in X$ we have $h\left(k^{-1}(j x)\right)=\left(h k^{-1}\right)(j x)$ by condition (2) of Definition (1.1). Hence $\varphi$ is regular at $\left(g g^{-1}, g x\right)$ and $1(g x)=\left(g g^{-1}\right) g x=g\left(g^{-1}(g x)\right)$. By the same argument, for general $h, k, j$ $\in G$ and $x \in X$ we have $h\left(k^{-1}(j x)\right)=\left(h k^{-1} j\right) x$. Therefore $\varphi$ is regular at $\left(g, g^{-1}(g x)\right)$ and $g\left(g^{-1}(g x)\right)=g x$. Hence $1(g x)=g x$ for general $g \in G, x \in$ $X$. As $G \times X \rightarrow G \times X,(g, x) \mapsto(g, g x)$ is dominant, $G \times X \cdot \rightarrow X,(g, x) \mapsto$ $g x$ is also dominant. Hence $g x$ is a general point in $X$. This shows that the condition (a) is satisfied. Conversely if the conditions (a), (b) are satisfied. Then rational map $G \times X \cdot \rightarrow X,(g, x) \mapsto g x$ is dominant. For general $g, h \in G$ and $x \in X$, we have $g^{-1}(h x)=\left(g^{-1} h\right) x$ by (b). $\left(g^{-1} g\right) x=1$. $x$ by (a). Therefore $\varphi$ is regular at $\left(g^{-1}, g x\right)$ and we have $g^{-1}(g x)=\left(g^{-1} g\right) x$ 
$=x$. If we denote $\psi_{1}(g, x)=g x, \psi_{2}(g, x)=g^{-1} x$, we can define $\psi_{2} \circ \psi_{1}$ and $\psi_{2} \circ \psi_{1}=$ Id. By the same argument $\psi_{1} \circ \psi_{2}=$ Id. Namely $\psi_{1}$ is birational.

We define similarly analytic group germ as in Definition (1.1). For the precise definition see Definition 1.1 in [U1].

But as we explained in [U2], even if we adopt an analogous definition in the analytic case, subtle problems arise in the definition of the birational automorphism group functor on the category of analytic spaces or in the definition of analytic subgroups in Bir $X$. The difference between the algebraic case and the analytic case comes from the existence of essential singularities in the latter case. First of all it is convenient to recall the definition of a meromorphic map between analytic sets ([K]).

Definition (1.12). Let $X$ be an analytic space. We say that a closed subset $A$ is rare if the restriction map $\Gamma\left(U, O_{X}\right) \rightarrow \Gamma\left(U-A, O_{X}\right)$ is injective for any open set $U$.

Definition (1.13). Let $X, Y$ be analytic spaces. A meromorphic map $f: X \leftrightarrow Y$ is a closed analytic set $\Gamma_{f} \subset X \times Y$ satisfying the following conditions:

(1) the restriction $p$ of the projection $p_{1}: X \times Y \rightarrow X$ to $\Gamma_{f}$ is proper,

(2) there exist a closed analytic subset $A$ of $X$ such that (i) $A$ and $p^{-1} A$ are rare and $p$ induces a biholomorphic isomorphism $\Gamma-p^{-1} A \simeq X$ $-A$.

Definition (1.14). Let $X$ be a complete algebraic variety. $\operatorname{Bim} X$ or $\operatorname{Bim} X^{\text {an }}$ is a group functor on the category of analytic spaces defined by:

$\operatorname{Bim} X(T)=\left\{f: T \times X \cdots T \times X \mid f\right.$ is a bimeromorphic map $\left(f\right.$ and $f^{-1}$ are meromorphic) commuting with the projection $p_{1}: T \times X \rightarrow T$, i.e. $p_{1}$ 。 $f=p_{1}$ such that there exists an open set $U$ with $t \times X \cap U \neq \varnothing$ for any $t \in T$ on which $f$ induces an isomorphism $\} / \sim$, where by the equivalence relation $\sim$ we identify meromorphic maps which coincide on a dense $T$ open set of $T \times X$ (i.e. the complement is rare).

Notice that the functor $\operatorname{Bim} X$ is independent of the choice of a complete model of $X$. Therefore for an algebraic variety $V$, we denote by $\operatorname{Bim} V$ the group functor $\operatorname{Bim} \bar{V}$ for a complete model $\bar{V}$ of $V$. When we consider $\operatorname{Bim} X$ for a complete variety $X$, we may assume by Hironaka's theorem that $X$ is projective and non-singular.

An analytic version of pseudo-operation is defined analogously but carefully. 
Definition (1.15). Let $G$ be an analytic group germ and $X$ a complete algebraic variety. A rational pseudo-operation $(G, \varphi, X)$ of the analytic group germ $G$ on $X^{\text {an }}$ is a meromorphic map $\varphi: G \times X^{\text {an }} \cdot \rightarrow X^{\text {an }}$ satisiying the conditions (1), (2) of Definition (1.2). When there is no danger of confusion, we denote $X^{\text {an }}$ by the same letter $X$.

Proposition (1.15) is generalized to

Proposition (1.16). Let $G$ be an analytic group and $X$ a complete algebraic variety. Then there is a 1-1 correspondence between the following:

(i) the set $\operatorname{Hom}_{g r}(G, \operatorname{Bim} X)$ of morphisms of group functors;

(ii) the set of rational pseudo-operations $(G, X)$.

Here in (ii) we have to identify isomorphic rational pseudo-operations.

We define a morphism of rational pseudo-operations similarly as in Definition (1.3).

Definition (1.17). Let $\left(G_{i}, \psi_{i}, X_{i}\right) i=1,2$ be rational pseudo-operations. A morphism $(\varphi, f):\left(G_{1}, X_{1}\right) \rightarrow\left(G_{2}, X_{2}\right)$ consists of a morphism $\varphi: G_{1} \rightarrow G_{2}$ of analytic group germs and a dominant meromorphic map $f: X_{1} \cdots X_{2}$ such that the diagram below is commutative:

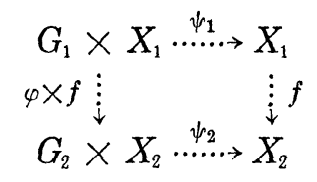

We say that $\left(G_{1}, X_{1}\right)$ and $\left(G_{2}, X_{2}\right)$ are isomorphic if there exist morphisms $\left(\varphi_{1}, f_{1}\right):\left(G_{1}, X_{1}\right) \rightarrow\left(G_{2}, X_{2}\right)$ and $\left(\varphi_{2}, f_{2}\right):\left(G_{2}, X_{2}\right) \rightarrow\left(G_{2}, X_{1}\right)$ such that $\varphi_{1} \circ \varphi_{2}$ and $\varphi_{2} \circ \varphi_{1}$ are identity around 1 and $f_{1} \circ f_{2}$ and $f_{2} \circ f_{1}$ are equivalent to the identities $1_{X_{2}}, 1_{X_{1}}$.

Definition (1.18). Let $G$ be an analytic group germ. A morphism $\varphi: G \rightarrow \operatorname{Bim} X$ of analytic group germ functors on the category of analytic spaces is a morphism $\varphi: U \rightarrow \operatorname{Bim} X$ of functors of an open neighbourhood $U$ of $1 \in G$ to $\operatorname{Bir} X$ such that the diagram

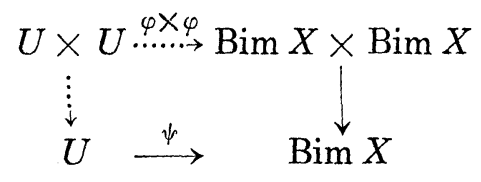

is commutative, where the vertical arrows are the composition laws. 
The same spirit of the proof of Proposition (1.6) leads us to

Proposition (1.19). Let $G$ be an analytic group germ and $X$ be $a$ complete algebraic variety. There is a 1-1 correspondence between the following:

(i) the set $\operatorname{Hom}_{g g}(G, \operatorname{Bim} X)$ of morphisms of analytic group germ functors (we identify two morphisms if they coincide in a neighborhood of 1);

(ii) open neighbourhoods $U$ of $1 \in G$ and rational pseudo-operations $(U, X)$.

Here in (ii) we have to identify isomorphic rational pseudo-operations. We have an analogue of Proposition (1.5).

Proposition (1.20). Let $X$ be a complete algebraic variety and $\varphi: G \rightarrow$ $\operatorname{Bim} X^{\text {an }}$ is a morphism of group functors of an analytic group $G$ to $\operatorname{Bim} X$. Then $\operatorname{Ker} \varphi$ is a closed analytic subgroup of $G$.

Definition (1.21). An analytic subgroup $\operatorname{Bim} X^{\text {an }}$ is a group subfunctor of $\operatorname{Bim} X^{\text {an }}$ representable by an analytic space.

Definition (1.22). Let $X$ be an algebraic variety, $G$ an algebraic group germ and $(G, X)$ an algebraic pseudo-operation. We can associate to $G$ an analytic group germ $G^{\text {an }}$. If $X$ is complete, then algebraic pseudooperation $G \times X \mapsto X$ is defined by the graph $\Gamma \subset G \times X \times X$. If we consider the associated analytic structure $\Gamma^{\text {an }} \subset G^{\text {an }} \times X^{\text {an }} \times X^{\text {an }}$, then it defines a rational speudo-operation $\left(G^{\text {an }}, X^{\text {an }}\right)=(G, X)^{\text {an }}$. We call $\left(G^{\text {an }}, X^{\text {an }}\right)$ the rational pseudo-operation associated to $(G, X)$. If $X$ is not complete, then we take a completion $\bar{X}$ and we get an algebraic pseudo-operation $(G, \bar{X})$ from $(G, X)$. Then we denote by $(G, X)^{\text {an }}$ or by $\left(G^{\text {an }}, X^{\text {an }}\right)$ the associated rational operation $(G, \bar{X})^{\text {an }}$.

The definition is justified since $(G, X)^{\text {an }}$ is determined up to isomorphism.

Let now $G$ be an algebraic group and $X$ be an algebraic variety. Let $\varphi: G \rightarrow \operatorname{Bir} X$ be a morphism of group functors on the category of $\mathbb{C}$ schemes. Then $\varphi$ is given by an algebraic pseudo-operation $(G, X)$ by Proposition (1.6), namely by a rational map $\psi: G \times X \mapsto X$. Let $\bar{X}$ be a completion of $X$. Then $\psi$ induces a rational map $G \times \bar{X} \leftrightarrow X$ which we denote also by $\psi$ hence an algebraic pseudo-operation $(G, \bar{X})$. Then the graph $\Gamma_{\psi} \subset$ $(G \times \bar{X}) \times \bar{X}$ of $\psi$ is a meromorphic map $\psi^{\text {an }}: G^{\text {an }} \times \bar{X}^{\text {an }} \leftrightarrow \bar{X}^{\text {an }}$. Therefore a 
morphism $\varphi^{\text {an }}: G^{\text {an }} \rightarrow \operatorname{Bim} \bar{X}^{\text {an }}$ of group functors on the category of analytic spaces by Proposition (1.16). It follows from the definition that $\varphi^{\text {an }}$ is independent of the choice of complete model $\bar{X}$ of $X$. When $G$ is an algebraic subgroup of $\operatorname{Bir} X$, then $G^{\text {an }} \rightarrow \operatorname{Bim}\left(\bar{X}^{\text {an }}\right)$ is an analytic subgroup by Propositions (1.20).

More generally by the same argument as above we can show

LEMMA (1.23). Let $X$ be a complete algebraic variety and $Y$ an analytic space. Then there is a 1-1 correspondence between the following:

(i) the set $\operatorname{Hom}(Y, \operatorname{Bim} X)$ of the morphism of functors;

(ii) $\{f: Y \times X \mapsto Y \times X \mid f$ bimeromorphic inducing an isomorphism of Zariski open sets $U, V$ of $Y \times X$ such that $y \times X \cap U, y \times X \cap V \neq \varnothing$ and $f$ commutes with the projection $Y \times X \stackrel{p_{1}}{\rightarrow} Y$; namely the diagram $Y \times X \cdots Y \times X$ ${ }^{\prime}{ }^{\prime}$

is commutative $\} / \sim$, where we identify by $\sim$ two bimeromorphic maps in (ii) if they coincide on a dense T-Zariski open set of $Y \times X$.

Let $X$ now be a smooth but not necessarily complete algebraic variety and $Y$ an algebraic variety. Let $f: Y \rightarrow \operatorname{Bir} X$ be a morphism of functors. It follows from Lemma (1.9) that the morphism $f$ is given by a $Y$-birational automorphism $\psi: Y \times X \mapsto Y \times X$. Let us take a complete model $\bar{X}$ of $X$. $\psi$ induces a $Y$-birational automorphism $\bar{\psi}: Y \times \bar{X} \cdot \rightarrow Y \times \bar{X}$. Let $\Gamma_{\bar{\psi}} \subset Y$ $\times \bar{X} \times_{Y} Y \times \bar{X}=Y \times \bar{X} \times \bar{X} \subset Y \times \bar{X} \times Y \times \bar{X}$ be the graph of $\bar{\psi}$. Then $\Gamma_{\bar{\psi}}$ defines a $Y^{\text {an }}$-pseudo-automorphism of the analytic space $(Y \times \bar{X})^{\text {an }} \cdot . \rightarrow$ $(Y \times \bar{X})^{\text {an }}$ and hence a morphism $Y^{\text {an }} \rightarrow \operatorname{Bim} \bar{X}^{\text {an }}$ of functors.

Definition (1.24). We denote this morphism $Y^{\text {an }} \rightarrow \operatorname{Bim} \bar{X}^{\text {an }}$ by $f^{\text {an }}$. The following trivial remark will be useful.

Lemma (1.25). Let $R$ be a ring of holomorphic functions over an domain $D \subset \mathbb{C}^{n}$. Assume that the ring $R$ is of finite type over $\mathbb{C}$ : there exist finitely may holomorphic functions $f_{1}, f_{2}, \cdots, f_{n}$ over $D$ such that $R=\mathbb{C}\left[f_{1}, f_{2}, \cdots, f_{n}\right]$. Let $\varphi: D \rightarrow \operatorname{Spec} R \subset \mathbb{A}^{n}$ be defined by $\varphi(x)=\left(f_{1}(x), f_{2}(x), \cdots, f_{n}(x)\right) \in \mathbb{A}^{n}$ for $x \in D$. Then the image $\varphi(D)$ is not contained in any closed algebraic subvariety of Spec $R$ except for Spec $R$ itself.

Proof. Let $F\left(x_{1}, x_{2}, \cdots, x_{n}\right) \in \mathbb{C}\left[x_{1}, x_{2}, \cdots, x_{n}\right]$ be a polynomial. If $F\left(f_{1}(x), f_{2}(x), \cdots, f_{n}(x)\right)=0$ for any $x \in D$, then $F\left(f_{1}, f_{2}, \cdots, f_{n}\right)=0$ in $R$.

Remark (1.26). In other words, by letting $K$ be the quotient field of 
$R$, the $K$-valued point $\left(f_{1}, f_{2}, \cdots, f_{n}\right) \in \operatorname{Spec} R \subset \mathbb{A}^{n}$ is the generic point of the scheme $\operatorname{Spec} R$.

Lemma (1.27). Let $A$ be an analytic manifold, $X$ an algebraic variety (defined over $\mathbb{C}$ ) and $f: A \rightarrow \operatorname{Bim} X$ a morphism of functors. Then, for any point $p \in A$, there exist an open neighborhood $U$ of $p$, an algebraic variety $B$, a morphism of functors $f^{\prime}: B \rightarrow \operatorname{Bir} X$ and a holomorphic map $g: U \rightarrow B^{\text {an }}$ such that (1) the image $g(U)$ is Zariski dense in the algebraic variety $B$ and (2) $f^{\prime \text { an }} \circ g=f$.

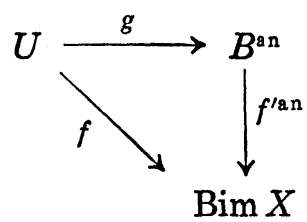

Proof. We may assume that $X$ is non-singular and projective. Let $f: A \rightarrow \operatorname{Bim} X$ be difined by a correspondence $A \times X \times X \supset Z$ (see Lemma (1.9)). The closed analytic subset $Z$ has the following property:

(1.27.i) The projection $p_{12}: A \times X \times X \rightarrow A \times X$ induces $Z \rightarrow A \times X$ which is an isomorphism outside of analytic subsets. As $X$ is projective, $p_{1}: A$ $\times X \times X \rightarrow A$ is contained in a projective fibration $A \times \mathbb{P}^{N} \rightarrow A$ for a certain integer $N$. It follows from the relative G.A.G.A. [G1] that the $A$-analytic subspace $Z \subset A \times X \times X \subset A \times \mathbb{P}^{N}$ is locally defined by some homogeneous polynomials with holomorphic coefficients with respect to $A$. More precisely there exist a neighbourhood $U$ of $p$, a ring $R$ of finite type over $\mathbb{C}$ consisting of holomorphic functions over $U$ and homogeneous polynomials $F_{i}\left(a ; X_{0}, X_{1}, \cdots, X_{N}\right)=F_{i}(a, X) \in R\left[X_{0}, X_{1}, \cdots, X_{N}\right] 1 \leqq i \leqq n$, $a \in A$ such that $Z=\left\{(a, x) \in A \times \mathbb{P}^{N} \mid F_{i}(a, x)=0\right.$ for $\left.1 \leqq i \leqq n\right\}$ over $U$. The homogeneous polynomials $F_{i}(a ; x) 1 \leqq i \leqq n$ define a $R$-scheme $\mathscr{Z} \subset \mathbb{P}_{R}$. We put $\mathscr{A}=\operatorname{Spec} R$. Then we have an inclusion $\mathscr{Z} \subset \mathscr{A} \times X \times X \subset \mathscr{A}$ $\times \mathbb{P}^{N}$ and the projection $p_{12}: \mathscr{A} \times X \times X \rightarrow \mathscr{A} \times X$ induces $q: \mathscr{Z} \rightarrow \mathscr{A} \times X$. We have also a natural analytic map $\varphi: U \rightarrow \operatorname{Spec} R=\mathscr{A}$ by Lemma (1.25). The diagram of natural maps

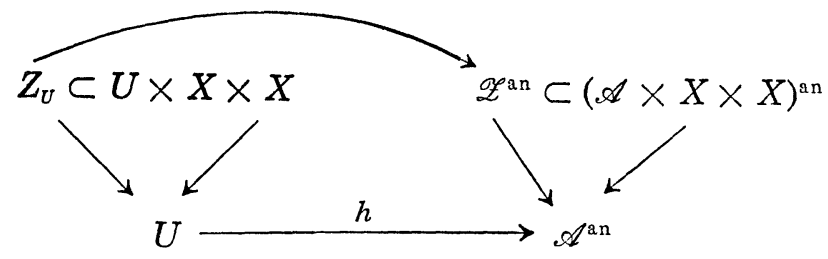


is a fibre product by construction, where we put $Z_{U}=Z \cap U \times X \times X$. It follows from (1.27.i) that for any point $a \in A$, there exists an open set $V_{a} \subset X$ such that $q^{-1}(a, x)$ consists of one point for $(a, x) \in a \times V_{a}$. Since by Lemma (1.25) the subset $\varphi(U) \subset \mathscr{A}$ is not contained in any proper closed algebraic subvariety of $\mathscr{A}$, it follows from [M] that the proper morphism $q: \mathscr{Z} \rightarrow \mathscr{A} \times X$ is birational. By the same argument we can show that the projection $p_{13}: \mathscr{A} \times X \times X \rightarrow \mathscr{A} \times X$ is birational. Namely $\mathscr{Z} \subset \mathscr{A} \times$ $X \times{ }_{\star} \mathscr{A} \times X=\mathscr{A} \times X \times X$ defines a birational automorphism of $\mathscr{A} \times X$ commuting with the projection.

Since the diagram is cartesian, the birational automorphism $\mathscr{Z}$ of $A \times X$ is biregular at $\left(g^{\prime}(p), x\right)$ for a general point $x \in X^{(1)}$. Thus we may assume that the correspondence $\mathscr{Z}$ gives an element of $\operatorname{Bir} X(\mathscr{A})$ and the Lemma is proved.

We proved the following Lemma in [U2].

Lemma (1.28). ([U2]), Lemma (1.8)). Let $X$ be a non-singular algebraic variety and $S$ an algebraic variety. Let $\varphi: S \rightarrow \operatorname{Bir} X$ be a morphism of functors on the category of $\mathbb{C}$-schemes. For any point $s \in S$, there exist an open neighbourhood $S^{\prime}$ of $s$, an algebraic variety $T$ and a morphism $\varphi^{\prime}: S^{\prime}$ $\rightarrow T$ such that $\varphi$ and $\varphi^{\prime}$ define the same equivalence relation on $S^{\prime}$ in the category of the algebraic varieties over $\mathbb{C}$. Here the last phrase that $\varphi$ and $\varphi^{\prime}$ define the same equivalence relation on $S^{\prime}$ in the category of the algebraic varieties over $\mathbb{C}$ means that for any algebraic variety $Z$ (defined over $\mathbb{C}$ ), $\varphi^{\prime}: S^{\prime}(Z) \rightarrow T(Z)$ and $S^{\prime}(Z) \longrightarrow S(Z) \stackrel{\varphi}{\rightarrow}$ Bir $X$ define the same equivalence relation on the set $S^{\prime}(Z)=\operatorname{Hom}\left(Z, S^{\prime}\right)$, i.e., the subset $W=\left\{(a, b) \in S^{\prime}(Z) \times\right.$ $\left.S^{\prime}(Z) \mid \varphi^{\prime}(a)=\varphi^{\prime}(b)\right\}$ of $S^{\prime}(Z) \times S^{\prime}(Z)$ coincides with the subset $W^{\prime}\{(a, b) \in$ $\left.S^{\prime}(Z) \times S^{\prime}(Z) \mid \varphi(a)=\varphi(b)\right\}$.

Lemma (1.29). Let $B$ be an algebraic variety, $X$ a smooth algebraic variety over $\mathbb{C}$ and $f^{\prime}: B \rightarrow \operatorname{Bir} X$ be a morphism of functors on the category of $\mathbb{C}$-schemes. Then there exist a non-empty Zariski open set $B^{\prime}$ of $B$, an. algebraic variety $C$, a morphism $h: C \rightarrow \operatorname{Bir} X$ of functors on the category of $\mathbb{C}$-schemes and a surjective morphism $q: B^{\prime} \rightarrow C$ of algebraic varieties such that (1) the restriction of $h$ on the category of the algebraic varieties is injective and (2) $f^{\prime}=h \circ q$.

Proof. It follows from Lemma (1.28) that there exist a non-empty Zariski open set $W$ of $B$, an algebraic variety $T$ and a morphism $\varphi^{\prime}: W$ $\rightarrow T$ such that $f^{\prime}$ and $\varphi^{\prime}$ define the same equivalence relation on $W$ on 
the category of the algebraic varieties. Then there exist a (not necessarily closed) algebraic subvariety $C$ of $T$ and a non-empty open set $U$ of $W$ such that $U$ and $C$ are non-singular and $\varphi^{\prime} \mid U$ is smooth so that $U \times{ }_{c} U$ is a union of algebraic varieties.

On the other hand since $\varphi^{\prime} \mid U=q: U \rightarrow C$ is faithful flat, the sequence

$$
\operatorname{Bir} X(C) \stackrel{q^{*}}{\longrightarrow} \operatorname{Bir} X(U) \underset{p_{2}^{*}}{\stackrel{p_{1}^{*}}{\longrightarrow}} \operatorname{Bir} X\left(U \times{ }_{c} U\right),
$$

is exact or $\operatorname{Im} q^{*}=\left\{x \in \operatorname{Bir} X(U) \mid p_{1}^{*} x=p_{2}^{*} x\right\}$ by [U2], Lemma (1.9.2), where $p_{i}: U \times{ }_{c} U \rightarrow U$ is the $i$-th projection. Let $y \in \operatorname{Bir} X(U)$ defines $f^{\prime} \mid U: U$ $\rightarrow \operatorname{Bir} X$. Then $p_{1}^{*} y=p_{2}^{*} y$ by the definition of $\varphi^{\prime}: W \rightarrow T$. Thus we get an element $z \in \operatorname{Bir} X(C)$ such that $q^{*} z=y . \quad h: C \rightarrow \operatorname{Bir} X$ defined by $z \in$ Bir $X(C)$ satisfies the condition.

The combination of Lemma (1.25) and (1.27) gives

Corollary (1.30). Let $A, X, f$ and $p$ be as in Lemma (1.27). Then there exist an open set $U$ of $A$, an algebraic variety $B$, a morphism of functors $f^{\prime}: B \rightarrow \operatorname{Bir} X$ on the category of $\mathbb{C}$-schemes and a holomorphic map $g: U \rightarrow B^{\text {an }}$ such that (1) the image $g(U)$ is Zariski dense in the algebraic variety $B$, (2) $f^{\prime \text { an }} \circ g=f$, (3) $U$ is dense in a neighbourhood of $p$ and (4) the restriction of $f^{\prime}$ on the category of algebraic varieties is injective.

The following lemma shows the birational unicity of $U$ in the corollary

LemMa (1.31). Let $A, X, f$ and $f^{\prime}$ be as in Lemma (1.27). Let $\tilde{U}$ be an open set of $A, \tilde{B}$ an algebraic variety, $\tilde{f}^{\prime}: \tilde{B} \rightarrow \operatorname{Bir} X$ be a morphism of functors on the category of $\mathbb{C}$-schemes and $\tilde{g}: \tilde{U} \rightarrow \tilde{B}^{\text {an }}$ such that (1) the image $\tilde{g}(\tilde{U})$ is Zariski dense in the algebraic variety $\tilde{B}$, (2) $\tilde{f}^{\prime \text { an }} \circ g=f$, (3) $\tilde{U}$ is dense in a neighbourhood of $p$ and (4) the restriction of $\tilde{f}^{\prime}$ on the category of algebraic varieties is injective. Then there exists an birational map $h: B \cdots \tilde{B}$ between $B$ of Corollary (1.31) and $\tilde{B}$ making the following diagram commutative:

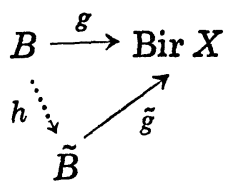

Namely we mean by the commutativity that the diagram above is commutative on the open set of $B$ on which $h$ is regular. 
Proof. Since $U$ and $\tilde{U}$ are dense at $p, W=U \cap \tilde{U}$ is a non-empty open set. The holomorphic maps $g$ and $\tilde{g}$ define $W \rightarrow B^{\text {an }}$ and $W \rightarrow B^{\text {an }}$ and $g(W)$ and $\tilde{g}(W)$ are dense respectively in $B$ and $B^{\prime}$. Hence we may assume $U=\tilde{U}$. Therefore we have

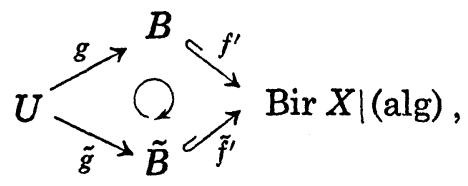

where (alg) denotes the category of the algebraic varieties over $\mathbb{C}$. It follows from [D] that the fibre product

$$
B \times \tilde{B} \stackrel{\varphi}{\longrightarrow} \operatorname{Bir} X
$$

is representable by a closed subscheme of $B \times \tilde{B}$ where $\varphi$ is defined by $\varphi(s, t)=f^{\prime}(s)\left(\tilde{f}^{\prime}(t)\right)^{-1} \in \operatorname{Bir} X$ for $(s, t) \in B \times \tilde{B}$. Therefore the fibre product $B \times_{\operatorname{Bir} X} \tilde{B} \mid$ (alg) is representable by a closed subvariety $C \subset B \times \tilde{B}$. The projections $B \times \tilde{B} \rightarrow B$ defines a morphism $q: C \rightarrow B$ of algebraic subvarieties, which is injective since $B$ and $\tilde{B}$ are subfunctors of $\operatorname{Bir} X \mid$ (alg). The variety $C=B \times_{\mathrm{Bir}} \tilde{B}$ contains the subset $g(U) \times \tilde{g}(U)(U=\tilde{U})$ and its image $q(g(U) \times \tilde{g}(U))=g(U)$ in $B$ is Zariski dense. In particular the subvariety $C \subset B$ is Zariski dense and hence $q: C \rightarrow B$ is birational. The same argument shows that the projection $B \times \tilde{B} \rightarrow \tilde{B}$ induces a birational $\operatorname{map} C \rightarrow \tilde{B}$.

Now we are ready to prove

TheOREm (1.32). Let $G$ be an analytic group germ, $X$ a complete algebraic variety and $(G, X)$ a rational pseudo-operation. Then there exist an algebraic group $H$ and an algebraic pseudo-operation $(H, X)$ and a morphism of rational pseudo-operations $(G, X) \rightarrow(H, X)^{\text {an }}$ inducing the identity on $X$.

Before we start the proof, we had better introduce a notion of algebraic pseudo-group.

Definition (1.33). An algebraic pseudo-group is a system $(G, m)$ satisfying the following condition:

(i) $G$ is an algebraic variety;

(ii) $m: G \times G \leftrightarrow G$ is a rational map;

(iii) the rational map $G \times G \cdot \rightarrow G \times G,(x, y) \mapsto(x, m(x, y))$ is dominant; 
(iv) the diagram

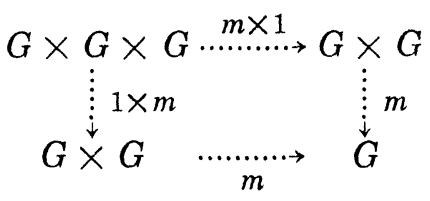

is commutative.

Definition (1.34). Let $G_{1}$ and $G_{2}$ be two algebraic pseudo-group. We say that $G_{1}$ and $G_{2}$ are birationally equivalent or equivalent if there exists a birational map $\varphi: G_{1} \cdots G_{2}$ such that $m_{2}(\varphi(x), \varphi(y))=\varphi\left(m_{1}(x, y)\right)$, where $m_{i}$ is the composition law of $G_{i}(i=1,2)$.

THEOREM (1.35) [W]. Every algebraic pseudo-group is equivalent to an algebraic group.

Definition (1.36). Let $G$ be an algebraic pseudo-group and $X$ be an algebraic variety. An algebraic pseudo-operation $(G, \psi, X)$ is a rational map $\varphi: G \times X \mapsto X$ satisfying the condition of Definition (1.2). The equivalence of algebraic pseudo-operations is defined as in Definition (1.3).

TheOREM (1.37) [W]. Let $(G, X)$ be an algebraic pseudo-operation of an algebraic pseudo-group $G$. Then there exist an algebraic group $\tilde{G}$ and an algebraic operation $(\tilde{G}, \tilde{X})$ equivalent to $(G, X)$.

Proof of the Theorem. The pseudo-rational operation $(G, X)$ defines a morphism of functors $X$ of an open neighbourhood $U$ of $1 \in G$ to $\operatorname{Bim} X$ by Proposition (1.19). Since the question is local, we may assume that the pseudo rational operation $(G, X)$ defines a morphism $f: G \rightarrow \operatorname{Bim} X$. We apply Corollary (1.30) for $A=G, p=1 \in G$ and $f: G \rightarrow \operatorname{Bim} X$. There exist an open set $U$ of $G$ dense in a neighbourhood of 1 , an algebraic variety $H$, a morphism of functors $f^{\prime}: H \rightarrow \operatorname{Bir} X$ and a holomorphic map $g: U \rightarrow H^{\text {an }}$ such that (1) the image $g(U)$ is Zariski dense in the algebraic variety $H$, (2) $f^{\prime \text { an }} \circ g=f$, (3) the restriction of $f^{\prime}$ on the category of the algebraic varieties is injective.

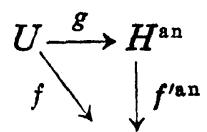

$\operatorname{Bim} X$

We show that $H \subset \operatorname{Bir} X \mid(\mathrm{Alg})$ has a natural structure of algebraic pseudogroup, Let us define a composition law on $H$. We may assume that the 
following commutative diagram exists

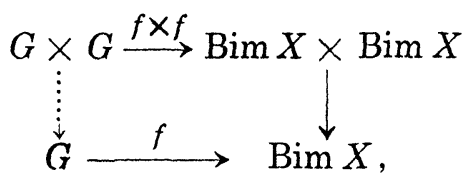

where the vertical arrows are composition laws. The diagram (1.38) gives

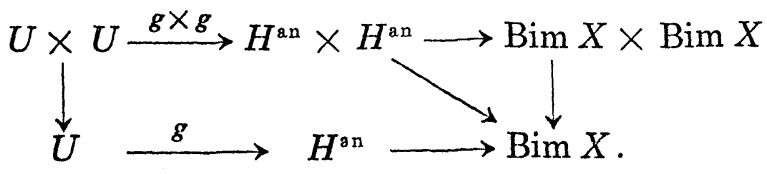

We want to show that there exist a rational map $\varphi: H \times H \cdots H$ such that $\varphi^{\text {an }}$ defines $H^{\text {an }} \times H^{\text {an }} \rightarrow \operatorname{Bim} X$. In fact, if we denote by $h: H \times H \rightarrow \operatorname{Bir} X$ the composite of the morphism $f^{\prime} \times f^{\prime}: H \times H \rightarrow \operatorname{Bir} X \times \operatorname{Bir} X$ and the composition law $\operatorname{Bir} X \times \operatorname{Bir} X \rightarrow \operatorname{Bir} X$, then it follows from Lemma (1.29) that there exist a Zariski open set $W$ of the algebraic variety $H \times H$, an algebraic variety $K$, a morphism $h^{\prime}: K \rightarrow \operatorname{Bir} X$ of the functors on the category of $\mathbb{C}$-schemes and a morphism $j: W \rightarrow K$ of algebraic varieties such that (1) the image $j(W)$ is Zariski dense in the algebraic variety $K$, (2) $h^{\prime} \circ j=h$ and (3) the restriction of $h^{\prime}$ on the category of algebraic varieties is injective.

The composite $\varphi_{1}: U \times U \rightarrow H^{\text {an }}$ of $g: U \rightarrow H^{\text {an }}$ and the composition law $U \times U \cdot \rightarrow U$ has the following properties.

(a) The image $\varphi_{1}(U \times U)$ is dense in the algebraic variety $H$ by Lemma (1.25).

(b) $U \times U$ is dense in a neighbourhood of $(1,1) \in G \times G$.

On the other hand, if we put $\tilde{U}=(g \times g)^{-1} W$ and denote the composite map $\tilde{U} \hookrightarrow U \times U \stackrel{g \times g}{\rightarrow} W \stackrel{h^{\prime}}{\rightarrow} K$ by $\varphi_{2}$, then we have the following properties.

(a) The image $\varphi_{2}(\tilde{U})$ is dense in the algebraic variety $K$ since the Zariski open set $W$ is dense in $H \times H$.

(b) $\tilde{U}$ is dense in a neighbourhood of $(1,1) \in G \times G$.

It follows from Lemma (1.31) that the inclusion $H \longrightarrow \operatorname{Bir} X \mid(\mathrm{alg})$ and $K \hookrightarrow \operatorname{Bir} X \mid($ alg) give birational correspondence of $H$ and $K$. Therefore there exist a rational map $\varphi: H \times H \cdots H$ such that the diagrams

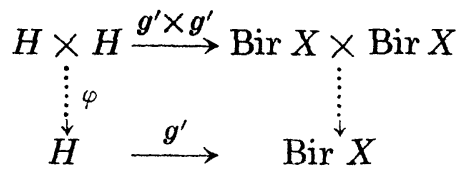


and

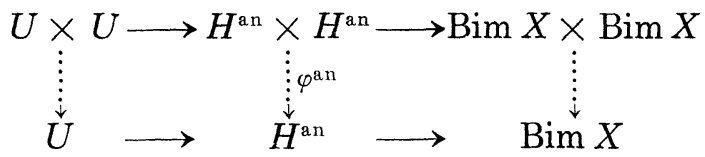

are commutative, where the right vertical arrows are the composition laws of $\operatorname{Bir} X$ and $\operatorname{Bim} X$.

Since $g(U)$ is dense in $H$, it follows from (1.39) and the proof of Lemma (1.25) that $\varphi: H \times H \leftrightarrow H$ is dominant. It follows from (1.39) that $H$ is an algebraic pseudo-group and we have a dominant rational map $H \times X . . \rightarrow H \times X$, which we denote by $(h, x) \mapsto(h, \mu(h, x))$. We have moreover a commutative diagram

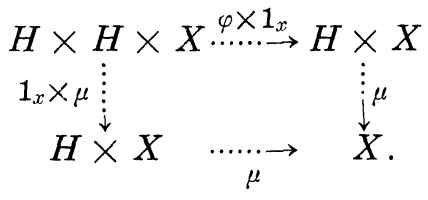

Namely the algebraic pseudo-group $H$ pseudo-operates on $X$. It follows from Theorem (1.37) that there exist an algebraic group $\tilde{H}$ and an algebraic operation $(\tilde{H}, \tilde{X})$ equivalent to $(H, X)$. In other words we have an equivalence $\alpha: H \leftrightarrow \tilde{H}$ of algebraic pseudo-group and a morphism $\beta: \tilde{H} \rightarrow \operatorname{Bir} X$ of group functors by Proposition (1.6) such that the diagram below is commutative:

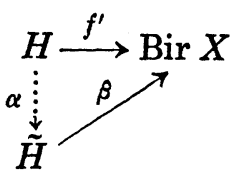

We thus get a morphism of an open subset $U^{\prime}$ dense in a neighbourhood of 1 of $U$ to $\tilde{H} ; \gamma: U^{\prime} \rightarrow \tilde{H}$ such that $\gamma(x) \gamma(y)=\gamma(x y)$ since $g(U)$ is dense in $H$ (cf. Lemma (1.25)). Since for a general $x, y \in U^{\prime}$ we have $\gamma(x)=$ $\gamma\left(x y, y^{-1}\right)=\gamma(x y) \gamma\left(y^{-1}\right), \quad \gamma$ can be extended to a regular map at $1 \in G$. Therefore we may assume that $U^{\prime}$ is a neighbourhood of $1 \in G$ and $\gamma: U^{\prime}$ $\rightarrow \tilde{H}$ with $\gamma(x, y)=\gamma(x) \gamma(y)$ for general $x, y \in U^{\prime} \times U^{\prime} . \quad(\tilde{H}, X)$ satisfies our requirement.

The following result is due to Painlevé [P].

Corollary (1.40). Let $\varphi: G \rightarrow \operatorname{Bim} X$ be a morphism of group functors 
of a (connected) analytic group $G$ to $\operatorname{Bim} X$. Then there exist an algebraic subgroup $i: H \hookrightarrow \operatorname{Bir} X$ and a morphism $\varphi^{\prime}: G \rightarrow H^{\text {an }}$ of analytic groups such that $\varphi=i^{\text {an }} \circ \varphi^{\prime}$.

Proof. Let $\varphi: G \rightarrow \operatorname{Bim} X$ be defined by a rational pseudo-operation $(G, X)$ by Proposition (1.16). Then by Theorem (1.32), we can find an algebraic group $H$, an algebraic pseudo-operation $(G, X)$ a local morphism $\gamma: G \rightarrow H$ of Lie groups such that $\left(\gamma, 1_{X}\right):(G, X) \rightarrow(H, X)^{\text {an }}$ is a morphism of rational pseudo-operations; $G \leftrightarrow H^{\text {an }} \rightarrow \operatorname{Bim} X$. Therefore if we take the universal covering group $\tilde{G}$ of $G$, we get a morphism $\tilde{r}: \tilde{G} \rightarrow H^{\text {an }}$ of Lie groups locally equivalent to $\gamma$. Let $H^{\prime}$ be an algebraic subgroup of $\operatorname{Bir} X$ such that the morphism $H \rightarrow \operatorname{Bir} X$ factors through $H^{\prime} \subset \operatorname{Bir} X$ (cf. Proposition (1.5)):

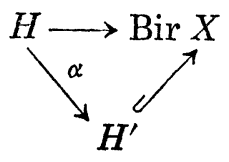

Therefore we get $\alpha \circ \tilde{\gamma}: \tilde{G} \rightarrow H^{\text {an }} \longrightarrow \operatorname{Bim} X$. Hence $\alpha \circ \tilde{\gamma}$ factors through $\varphi: G \rightarrow \operatorname{Bim} X$.

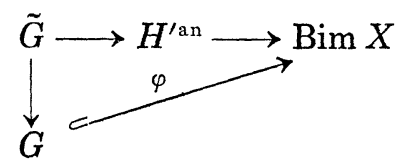

The algebraic subgroup $H^{\prime}$ satisfies the conditions of Corollary (1.40).

\section{Part II. Algebraic differential equations}

\section{§1. Preliminaries}

In the study of algebraic differential equations, algebraic varieties defined over not necessarily algebraically closed fields appear. We are always in characteristic 0 but the algebraic non-closedness often makes the argument delicate. The analyst may naively imagine with an algebraic variety a set of the common zeros of certain polynomials. But we have to clarify the definition.

Definition (1.1). Let $K$ be a field. An algebraic variety over $K$ or $K$-algebraic variety is an irreducible and reduced $K$-scheme of finite type 
over Spec $K$.

We work always in characteristic 0. Even under this assumption for a field extension $L \supset K$ and a $K$-algebraic variety $X$, the base change $X \otimes_{K} L$ is not always an $L$-algebraic variety. We know that in characteristic 0 , each irreducible component of $X \otimes_{K} L$ is an $L$-algebraic variety for sufficiently big $L$ (see for example, E.G.A. IV, 4.3.).

Definition (1.2). Let $X$ be an algebraic variety over a field $K$. We say that $X$ is absolutely irreducible (in E.G.A. géométriquement intègre) if for any field extension $L \supset K, X \otimes_{K} L$ is an algebraic variety over $L$.

We know (cf. E.G.A. Chap IV, 4.5).

Proposition (1.3). Let $X$ be an algebraic variety defined over $K$. Then $X$ is absolutely irreducible if and only if there exists an algebraically closed extension $L \supset K$ such that $X \otimes_{K} L$ is an algebraic variety.

As we heavily use the algebro-geometric language in the following discussion, it would be useful to explain certain basic conventions so that the paper is accessible for analysts. Let $V$ be an algebraic variety defined over $k$ or we say sometimes that $V$ is a $k$-algebraic variety. All the ring we consider are commutative with the unit element. Let $R$ be a ring containing the field $k$. An $R$-rational point or $R$-valued point of $V$ is a morphism Spec $R \rightarrow V$ of $k$-schemes. In particular a $k$-valued point Spec $k \rightarrow V$ is determined by its image and hence we say that the image $x \in V$ of $\operatorname{Spec} k$ is a $k$-valued point. More generally, for a field $K \supset k$ since Spec $K$ consists of a point, the image Spec $K \rightarrow V$ of a $K$-valued point is sometimes called by abuse of language a $K$-valued point; if $x$ is the image of Spec $K, K$ is an extension of the residue field $k(x)$. Let $S \supset R$ be a over ring. Then the inclusion $S \supset R$ defines the morphism Spec $S \rightarrow \operatorname{Spec} R$ and hence we have an $S$-valued point Spec $S \rightarrow V$ by the composition of the above two morphisms. For this reason an $R$-valued point is naturally considered as an $S$-valued point for $S \supset R$. Let now $f: V \rightarrow W$ be a morphism of $k$-algebraic variety and $g$ : Spec $R \rightarrow V$ an $R$ valued point. Then we get an $R$-valued point $f \circ g$ : Spec $R \rightarrow W$. Let $(G, \varphi, X), \varphi: G \times{ }_{k} X \rightarrow X$ be a $k$-algebraic operation of a $k$-algebraic group $G$ over $k$-algebraic variety $X$. For $g \in G, x \in X$, we denote by $g x$ the image $\varphi(g, x)$ (cf. Part I). Let $f:$ Spec $R \rightarrow G$ be an $R$-valued point and $x \in X$ be a $k$-valued point. Therefore we get an $R$-valued point 


$$
\begin{aligned}
& \text { Spec } R \rightarrow G \times_{k} X \stackrel{\varphi}{\longrightarrow} X \quad \text { of } X \text {. } \\
& a \longmapsto(f(a), x)
\end{aligned}
$$

This rather trivial remark is useful when we try to integrate differential equations.

Let $D \subset \mathbb{C}$ be a domain, $R$ a ring of holomorphic functions and $K$ the quotient field of $R$. In the following sections, we consider field extensions $L$ which is not necessary of finite type over $K$. But $L$ is the quotient field of a ring $S$ of holomorphic functions on a subdomain $D_{1}$ of $D$.

A typical example of such $L$ is a finite algebraic extension of $K$. In fact let $L \supset K$ be a finite algebraic extension. Then $L$ is a simple extension: $L=K(\alpha)$. Let $\alpha$ satisfy an irreducible algebraic equation $\alpha^{n}+a_{1} \alpha^{n-1}$ $+\cdots+a_{n}+0, a_{i} \in K(1 \leqq i \leqq n)$. Iet $D^{\prime} \subset D$ be a subdomain such that the $a_{i}$ are regular on $D^{\prime}$. Let $f$ be a multivalued function on $D^{\prime}$ satisfying

$$
f^{n}+a_{1} f^{n-1}+\cdots+a_{n}=0 .
$$

Namely $f$ is an algebroid function. If we take a subdomain $D_{1} \subset D^{\prime}$ such that $f$ decomposes into $n$-single valued functions, then by letting $f$ be a branch of $f$ on $D_{1}$, we get that $L=K(\alpha)$ is $K$-isomorphic to $K\left(f_{1}\right)$. Therefore $K\left(f_{1}\right)$ is the quotient field of $R\left[a_{1}, a_{2}, \cdots, a_{n}, f_{1}\right]$. The latter is a ring of holomorphic functions on $D_{1}$.

We identify a holomorphic function on $D$ with its restriction on a sub-domain $D_{1}$. Therefore often we do not make the subdomain $D_{1}$ precise. It is convenient to use an algebraic closure $\bar{K}$ of $K$ in the following discussions but we should be careful because this field is not of the above type. Hence we have to justify this usage. In fact, every time we use $\bar{K}$, it is sufficient to consider a sufficiently big finite algebraic extension of $K$ and this field is of above type as we saw above.

A typical example is as follows. Let $V \subset \mathbb{A}_{K}^{n}$ be an $K$-affine algebraic variety. Let us decompose $V \otimes_{K} \bar{K}$ into the union of irreducible varieties (hence absolutely irreducible varieties) over $\bar{K}$. As every component of $V \otimes_{K} \bar{K}$ is defined over a finite algebraic extension of $K$, the decomposition is done over a finite algebraic extension of $K$.

For this reason, we may use the algebraic closure $\bar{K}$ in such situations as in the above examples.

Let $V$ be an algebraic variety defined over $\mathbb{C}$ and $D$ a domain of $\mathbb{C}$. When there is no danger of confusion, we denote the analytic space $V^{\text {an }}$ 
associated with $V$ also by $V$. For example a holomorphic map or a holomorphic curve $F: D \rightarrow V^{\text {an }}$ with be simply denoted by $F: D \rightarrow V$. Let us now assume $V$ to be affine so that we have an embedding $V \subset \mathbb{A}_{\mathrm{c}}^{n}=$ $\mathbb{A}^{n}$. Let $F: D \rightarrow V \subset \mathbb{A}^{n}$ be defined by bolomorphic functions $\left(f_{1}, f_{2}, \cdots, f_{n}\right)$ on $D: F(t)=\left(f_{1}(t), f_{2}(t), \cdots, f_{n}(t)\right) \in V \subset \mathbb{A}^{n}$ for $t \in D$. Two interpretations are possible. The first one is given above: $F: D \rightarrow V$ is a holomorphic curve on an algebraic variety $V$. The second one is algebro-geometric. Let $K$ be the field $\mathbb{C}\left(f_{1}, f_{2}, \cdots, f_{n}\right)$ of meromorphic functions on $D$.

The map $F$ defines a homomorphism $\mathbb{C}\left[X_{1}, X_{2}, \cdots, X_{n}\right] \rightarrow \mathbb{C}\left[f_{1}, f_{2}, \cdots, f_{n}\right]$ $=R$ of $\mathbb{C}$-algebras. Therefore a morphism $f:$ Spec $\mathbb{C}\left[f_{1}, f_{2}, \cdots, f_{n}\right] \rightarrow$ Spec $\mathbb{C}\left[X_{1}, X_{2}, \cdots, X_{n}\right]=\mathbb{A}_{\mathrm{C}}^{n}$ of $\mathbb{C}$-algebraic varieties. This morphism factors through $V \subset \mathbb{A}_{\mathrm{c}}^{n}$ giving an $R$-valued point $\operatorname{Spec} R \rightarrow V$. Since $K \supset R$, we get an $K$-valued point Spec $K \rightarrow V$, which we denote by $P(F)$ and call the $K$-valued point associated with $F$. We say also that $F: D \rightarrow V$ is a $K$ valued point. Conversely given a $K$-valued point $Q=\left(g_{1}(t), g_{2}(t), \cdots, g_{n}(t)\right)$ $\in V \otimes_{\mathrm{c}} K$ with $g_{i}(t) \in K, 1 \leqq i \leqq n$. Then there is a subdomain $D_{1} \subset D$ such that the $g_{i}(t)(1 \leqq i \leqq n)$ are regular on $D_{1}$ and hence defines a holomorphic curve $G: D_{1} \rightarrow V$. If the variety $V$ is not affine, using an affine covering we define the $K$-valued point $P(F)$ for a holomorphic curve $F: D \rightarrow V$.

Let $D_{1} \subset D_{2}$ be domains of $\mathbb{C}$ and $F_{i}: D_{i} \rightarrow V$ be a holomorphic curve $(i=1,2)$. We say that $F_{2}$ is equivalent to $F_{1}$ and write $F_{2} \sim F_{1}$ if $F_{1}$ is the restriction of $F_{2}$ onto $D_{1}$. We have proved

Propositjon (1.4). Let $L \supset \mathbb{C}$ be a field of meromorphic functions on a domain $D$. Let $V$ be an algebraic variety over $\mathbb{C}$. Then there is a 1-1 correspondence between the following.

(i) $\left\{F: D^{\prime} \rightarrow V \mid F\right.$ is a holomorphic curve on a subdomain $D^{\prime} \subset D$ depending on $F$ such that $P(F)$ is a L-valued point / equivalence relation generated by $\sim$.

(ii) L-valued points of $V$.

Remark (1.5). In Proposition (1.4), we do not assume $V$ to be affine, since the question is local and hence reduced to the affine case discussed above.

Definition (1.6). Let $p$ : Spec $L \rightarrow V$ be an $L$-valued point. The corresponding holomorphic curve $F: D^{\prime} \rightarrow V$ is denoted by $F(P)$. For an algebraic variety $V$, we have seen in Proposition (1.4) a nice 1-1 corre- 
spondence between the holomorphic curves $F: D^{\prime} \rightarrow V^{\text {an }}$ and $L$-rational points of $V$. A similar correspondence exists between morphisms $F: D^{\prime}$ $\rightarrow \operatorname{Bim} V^{\text {an }}$ and morphisms $\operatorname{Spec} L \rightarrow \operatorname{Bir} V$. Since $\operatorname{Bim} V^{\text {an }}$ and $\operatorname{Bir} V$ are functors, we should argue carefully. In fact, let $V$ be a projective algebraic variety defined over $\mathbb{C}$ and $D$ be a domain of $\mathbb{C}$.

Let $R$ be a $\mathbb{C}$-algebra. $R$-rational point of $\operatorname{Bir} V$ is a morphism Spec $R \rightarrow \operatorname{Bir} V$ of functors. Let $F: D \rightarrow \operatorname{Bim} V^{\text {an }}$ be a morphism of functors on the category of the complex analytic spaces. Replacing $D$ by a subdomain if necessary, we may assume by Lemma (1.27) that there exist an algebraic variety $W$ a morphism $f^{\prime}: B \rightarrow \operatorname{Bir} V$ and a holomorphic map $g: D \rightarrow B^{\text {an }}$ such that (1) the image $g(D)$ is Zariski dense in the algebraic variety $W$ and (2) $f^{\prime a n} \circ g=F$. It follows from the argument above that $g$ determines a $K$-valued point Spec $K \rightarrow B$ hence a $K$-valued point Spec $K$ $\rightarrow B \rightarrow$ Bir $V$, where $K$ is an appropriate field of meromorphic functions on $D$. We denote this $K$-valued point on the functor Bir $V$ by $P(F)$ and call the associated $K$-valued point to $F$. We say also that $F: D \rightarrow \operatorname{Bim} V^{\text {an }}$ is a $K$-valued point. Conversely let $L$ be a field of meromorphic functions on a domain $D$ and $\operatorname{Spec} L \rightarrow \operatorname{Bir} V$ be a $L$-valued point. The $L$-valued point Spec $L \rightarrow \operatorname{Bir} V$ is given by an $L$-birational automorphism of $V \otimes_{\mathrm{C}} L$, or by its graph $\Gamma \subset\left(V \otimes_{\mathrm{c}} L\right) \times\left(V \otimes_{\mathrm{c}} L\right)=\left(V \times_{\mathrm{C}} V\right) \otimes_{\mathrm{c}} L$ (cf. Lemma (1.9)). Therefore there exists a $\mathbb{C}$-algebra of finite type $R$ such that the graph $\Gamma$ is defined over $R$ : there exists an algebraic subvariety $\Gamma_{R} \subset(V \times V) \otimes_{\mathrm{C}}$ Spec $R$ such that $\Gamma=\Gamma_{R} \otimes_{R} K$. We may further assume that $\Gamma_{R} \subset$ $(V \times V) \otimes_{\mathrm{c}}$ Spec $R$ defines an Spec $R$-birational automorphism of Spec $R$ $\otimes_{\mathrm{C}} V$. Thus we get a morphism $\operatorname{Spec} R \rightarrow \operatorname{Bir} V$. Let $R=\mathbb{C}\left[f_{1}, f_{2}, \cdots, f_{n}\right]$. We get finally $D \rightarrow(\operatorname{Spec} R)^{\text {an }} \rightarrow \operatorname{Bim} V^{\text {an }}$.

Let $D_{1} \subset D_{2}$ be domains of $\mathbb{C}$ and $F_{i}: D_{i} \rightarrow$ Bim $V$ be a morphism of functors. We say that $F_{2}$ is equivalent to $F_{1}$ and write $F_{2} \sim F_{1}$ if $F_{1}$ is the restriction of $F_{2}$ onto $D_{1}$. Leaving the reader the detail because it is only a formal checking, we have thus proved

Proposition (1.7). Let $V$ be an algebraic variety over $\mathbb{C}, L \supset \mathbb{C}$ be a field of meromorphic functions. Then there is a 1-1 correspondence between the following.

(i) $\left\{F: D^{\prime} \rightarrow \operatorname{Bim} V \mid F\right.$ is a morphism of functors of a subdomain $D^{\prime} \subset D$ depending on $F$ such that $P(F)$ is L-rational\}/ equivalence relation generated by $\sim$. 
(ii) L-rational points of Bir $V$.

Definition (1.8). Let $p: \operatorname{Spec} L \rightarrow \operatorname{Bir} V$ be an $L$-rational point. Then the corresponding morphism $D^{\prime} \rightarrow \operatorname{Bim} V$ is denoted by $F(P)$.

The first statements in Propositions (1.4) and (1.7) are analytic nature and the second statements are algebraic. The conditions on a differential equation is given in the analytic language. In our study of the differential equations, we translate them by Propositions (1.4) and (1.7) into the algebro-geometric language. Using the technique of algebraic geometry, we study the properties of the differential equation and finally we translate the results into the analytic language by Propositions (1.4) and (1.7).

We shall study systems of Pfaffian differential equations over manifolds. We need only the simplest systems.

Definition (1.9). Let $M$ be a (complex) analytic manifold. We denote by $T_{M}$ the tangent bundle of $M$ and by $\Theta_{M}$ the sheaf of its sections. But we do not distinguish strictly a vector bundle and the locally free sheaf of its sections. A regular system of Pfaffian differential equations of corank 1 or of rank $\operatorname{dim} M-1$ over $M$ is a subbundle $L$ of rank 1 of the tangent bundle $T_{M}$ so that we have an exact sequence

$$
0 \longrightarrow L \longrightarrow T_{M} \longrightarrow T_{M} / L \longrightarrow 0 \quad \text { with } T_{M} / L
$$

locally free $O_{M}$-module. Considering the dual $\Omega_{M}^{1}$ of $T_{M}$, we can show this is equivalent to giving a locally free subsheaf $\mathscr{E}$ of $\operatorname{rank} \operatorname{dim} M-1$ such that $\Omega_{M}^{1} / \mathscr{E}$ is an invertible sheaf:

$$
0 \longrightarrow \mathscr{E} \stackrel{u}{\longrightarrow} \Omega_{M}^{1} \longrightarrow \Omega_{M}^{1} / \mathscr{E} \longrightarrow 0 .
$$

Let $F: D \rightarrow M$ be a holomorphic curve of a domain $D \subset \mathbb{C}$. We say that $F$ is a solution of the system of Pfaffian differential equations if the composite map $F^{*} \stackrel{\mathscr{E}}{F^{*} u} \rightarrow F^{*} \Omega_{M}^{1} \rightarrow \Omega_{D}^{1}$ is 0 , where $F^{*} \Omega_{M}^{1} \rightarrow \Omega_{D}^{1}$ is the natural map. When $\mathscr{E}$ is generated by 1 -forms, $w_{1}, w_{2}, \cdots, w_{n}$, we call (1.10) or (1.11), a system of Pfaffian differential equations $w_{1}=0, w_{2}=0, \cdots, w_{n}=0$.

We had better generalized Definition (1.9).

Definition (1.12). A system of Pfaffian differential equation of corank 1 , or of rank $\operatorname{dim} M-1$ is a coherent subsheaf (1.13) $0 \rightarrow L \rightarrow T_{M}$ such that there exists a non-empty open set $M^{0} \subset M$ over which the injection (1.13) 
induces a regular system of Pfaffian differential equations of corank 1 . By duality a coherent subsheaf $0 \rightarrow \mathscr{E} \rightarrow \Omega_{M}^{1}$ such that $\mathscr{E}$ is locally free of rank $\operatorname{dim} M-1$ over a non-empty open set defines a system of Pfaffian differential equations of corank 1 . If there is no danger of confusion, we do not make the open set $M^{0}$ precise.

A simple but important example of such a Pfaffian system arises when we consider a group operation. Let $G$ be a algebraic group, $X$ a nonsingular algebraic variety and $(G, \varphi, X), \varphi: G \times X \rightarrow X$ be an algebraic operation over $\mathbb{C}$. We denote $\varphi(g, x)$ by $g \cdot x$ or by $g x$ for $g \in G, x \in X$. Let $F: D \rightarrow G$ be a holomorphic curve whose associated point $P(F)$ is $K$ valued for a certain field $K$ of meromorphic functions. By enlarging $K$, let us assume that if $f(t) \in K$, then the derived function $f^{\prime}(t) \in K$. Then we can define a $K$-vector field $\Theta(F,(G, X))=\Theta(F)$ on $X \otimes_{\mathrm{c}} K$ or an element of $H^{\circ}\left(X \otimes_{\mathrm{c}} K, \Theta_{X \otimes K / K}\right)$. In fact we construct $\Theta(F)$ locally. Let $U \subset X$ be an affine open set. Let $U=\operatorname{Spec} A$ with $A=\mathbb{C}\left[z_{1}, z_{2}, \cdots, z_{m}\right]$. We consider $U \subset \mathbb{A}^{m}$ by $\mathbb{C}\left[Z_{1}, Z_{2}, \cdots, Z_{m}\right] \rightarrow \mathbb{C}\left[z_{1}, z_{2}, \cdots, z_{m}\right], Z_{i} \mapsto z_{i}(1 \leqq i \leqq m)$. For $f \in H^{\circ}\left(U, O_{U}\right)$ and $t \in D$, we put

$$
\Theta(F)(f(x))=\lim _{h \rightarrow 0} \frac{f\left(F(t+h) F^{-1}(h) x\right)-f(x)}{h}
$$

considering $t \in D$ as a variable. Then $\Theta(F): H^{0}\left(U, O_{U}\right) \rightarrow H^{0}\left(U \otimes_{\mathrm{c}} K, O_{U \otimes_{\mathrm{C}} K}\right)$ is a $\mathbb{C}$-derivation. Let us check that $\Theta(F)(f(x))$ is in fact in $H^{0}\left(U \otimes_{\mathrm{c}} K\right.$, $\left.O_{U \otimes_{\mathrm{C}} K}\right)$. Let $\varepsilon^{2}=0$. Then for an analytic function $a(t) \in K a(t+\varepsilon)=a(t)$ $+a^{\prime}(t) \varepsilon \in K[\varepsilon]$ since $a^{\prime}(t)$ is in $K$. Let $F: D \rightarrow G$ be given locally by $t \mapsto$ $\left(f_{1}(t), f_{2}(t), \cdots, f_{m}(t)\right) \in U \subset \mathbb{A}^{m}$. On a suitable subdomain $D^{\prime} \subset D, F$ defines $F(t+h): D \rightarrow G, t \mapsto\left(f_{1}(t+h), f_{2}(t+h), \cdots, f_{m}(t+h)\right)$ for $h \in \mathbb{C}$ near the 0 . If we consider $F(t+h) \bmod h^{2}$. We get a morphism $P(F(t+h)$ : Spec $K[\varepsilon] \rightarrow G$. We have a natural map $q$ : Spec $K[\varepsilon] \rightarrow \operatorname{Spec} K$. We thus get a map $(\mathrm{Id}, q):$ Spec $K[\varepsilon] \rightarrow \operatorname{Spec} K[\varepsilon] \otimes_{\mathrm{C}} \operatorname{Spec} K$. Composing with

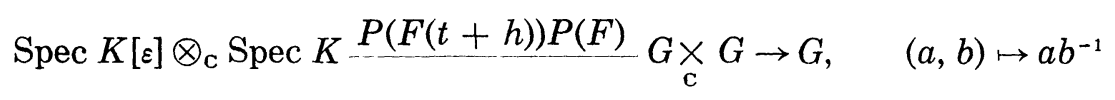

we get a morphism $\psi:$ Spec $K[\varepsilon] \rightarrow G$ and therefore we get

$$
\alpha: \operatorname{Spec} K[\varepsilon] \times_{c} X \rightarrow G \times_{\mathrm{c}} X \rightarrow X
$$

Since set-theoretically $\psi_{\text {red }}$ : Spec $K[\varepsilon]_{\text {red }}=\operatorname{Spec} K \rightarrow G_{\text {red }}=G$ factors through 1 , for the open set $U$ above, we get $\alpha_{U}$ : Spec $K[\varepsilon] \times_{\mathrm{c}} U \rightarrow U$. Then 
$f\left(F(t+h) F^{-1}(t) x\right) \bmod h^{2}$ is equal to $\alpha_{U}^{*} f$ and hence $\Theta(F)(t(x))=$ the coefficients of $\varepsilon$-term of $\alpha_{U}^{*} f \in H^{0}\left(\operatorname{Spec} K[\varepsilon] \times_{\mathrm{c}} U, O\right)=A \otimes_{\mathrm{c}} K+A \otimes_{\mathrm{c}} K \varepsilon$. Therefore $\Theta(F)(t(x))$ is in $A \otimes_{\mathrm{C}}$ Spec $K . \quad \Theta(F)$ is evidently $\mathbb{C}$-linear and we can check directly $\Theta(F)(f g)=\Theta(F)(f) . g+f . \Theta(F)(g)$ for $f, y \in H^{0}\left(U, O_{U}\right)$. Extending the derivation $\Theta(F): H^{0}\left(U, O_{U}\right) \rightarrow H^{0}\left(U \otimes_{\mathrm{C}} K, O_{U \otimes_{\mathrm{C}} K}\right)$ to $H^{0}\left(U, O_{U}\right)$ $\otimes_{\mathrm{C}} K=H^{0}\left(U \otimes_{\mathrm{c}} K, O_{U \otimes_{\mathrm{C}} K}\right) \rightarrow H^{0}\left(U \otimes_{\mathrm{C}} K, O_{U \otimes_{\mathrm{C}} K}\right)$ by sending $1 \otimes K$ to 0 . We get a $K$-derivation $H^{0}\left(U \otimes_{\mathrm{C}} K, O_{U \otimes_{\mathrm{C}} K}\right)$ which we again denote by $\Theta(F)$. We call $\Theta(F)$ the vector field associated with $F$. Another interpretation of the formula (1.14) is: $\Theta(F)$ is a vector field on $D \times X$ if we put $\Theta(F)(f(x))$ $=\lim _{h \rightarrow 0}\left(f\left(F(t+h) F^{-1}(h) x\right)-f(x)\right) / h$ for any holomorphic function on $D^{\prime}$ $\times U$ where $D^{\prime}$ is a subdomain of $D$. Therefore we get a non-vanishing vector field $\partial / \partial t+\theta(F)$ on $D \times X$ and

$$
0 \rightarrow O_{D \times x}\left(\frac{\partial}{\partial t}+\Theta(F)\right) \rightarrow T_{D \times X}
$$

is a regular system of Pfaffian differential equations of rank $\operatorname{dim} X$. It follows from the definition that the holomorphic curve $H: D \rightarrow D \times X, t \rightarrow$ $(t, F(t) x)$ is a solution of the system of Pfaffian differential equations $(\operatorname{Pf}(F))$ for any point $x \in X$. Jn fact the holomorphic curve $H$ defines a vector field $X_{(t, F(t) x)} \varphi=\lim _{h \rightarrow 0}(\varphi(t+h, F(t+h) x)-\varphi(t, F(t) x)) / h$ at $(t, F(t) x)$, for a holomorphic function $\varphi$ at $(t, F(t) x)$. We have $X_{(t, F(t) x)} t=1$. Let $\varphi$ be a holomorphic function on a neighbourhood of $F(t) x=y \in X$. Then $X_{(t, F(t) x)} \varphi=\lim _{h \rightarrow 0}(\varphi(F(t+h) x))-\varphi(F(t) x) / h=\lim _{h \rightarrow 0}\left(\varphi\left(F(t+h) F(t)^{-1} y\right)-\right.$ $\varphi(y)) / h=\Theta(F)(y)(\varphi)$. Hence $H$ defines the vector field $\partial / \partial t+\Theta(F)$ and $H$ is a solution. The above argument shows that any solution $D \rightarrow D \times X$, $t \mapsto(t, a(t))$ of the system $\operatorname{Pf}(\theta)$ is given by $H$ varying $x \in X$.

We call $\operatorname{Pf}(F)$ the system of Pfaffian differential equation associated with $F$ and $(G, X)$.

Definition (1.15). If there exist a subfield $L \supset \mathbb{C}$ of $K$ and a vector field $\theta$ on $X \otimes_{\mathrm{C}} L$ or $\theta \in H^{0}\left(X \otimes_{\mathrm{c}} L, \Theta_{X \otimes_{\mathrm{C}} L / L}\right)$ such that $\theta \otimes_{L} K \in H^{0}\left(X \otimes_{\mathrm{c}} K\right.$, $\left.\Theta_{X \otimes_{\mathrm{C}} K / K}\right)$ coincides with $\Theta(F)$, then we say that the system $\operatorname{Pf}(F)$ of Pfaffian differential equations are defined over $L$ or the coefficients of the system $\operatorname{Pf}(F)$ are in $L$.

A particular case of the above example is given by the operation $(G, G)$ of an algebraic group $G$ on itself from the left. This appears in $\S 2$.

In our study of differential equations, the following situation appears very often. Let $X=\operatorname{Spec} \mathbb{C}\left[x_{1}, x_{2}, \cdots, x_{n}\right]$ be an affine algebraic variety, 
$D \subset \mathbb{C}$ a domain and $K$ a field of meromorphic functions on $D$ containing all the constant functions. We denote by $X_{\text {reg }}$ the set of the non-singular points of $X$. The coordinate on $D$ is denoted by $t$. Let $\theta$ be a rational vector field on the algebraic variety $X \otimes_{\mathrm{C}} K$ over $K$, namely a $K$-derivation $\theta$ : quotient field of $\mathbb{C}\left[x_{1}, x_{2}, \cdots, x_{n}\right] \otimes_{\mathbb{C}} K \rightarrow$ quotient field of $\mathbb{C}\left[x_{1}, x_{2}, \cdots\right.$, $\left.x_{n}\right] \otimes_{\mathrm{c}} K . \quad \theta$ is given by $\theta\left(x_{i}\right)=\left(F_{i}\left(t ; x_{1}, x_{1}, \cdots, x_{n}\right)\right) /\left(G_{i}\left(t ; x_{1}, x_{2}, \cdots, x_{n}\right)\right)$ with $F_{i}\left(t ; X_{1}, X_{2}, \cdots, X_{n}\right), G_{i}\left(t ; X_{1}, X_{2}, \cdots, X_{n}\right) \in K\left[X_{1}, X_{2}, \cdots, X_{n}\right]$ such that $G_{i}\left(t ; x_{1}, x_{2}, \cdots, x_{n}\right) \neq 0$ for $1 \leqq i \leqq n$. Therefore the vector field $\partial / \partial t+$ $\sum_{i=1}^{n}\left(F_{i}\left(t, X_{1}, \cdots, X_{n}\right)\right) /\left(G_{i}\left(t, X_{1}, \cdots, X_{n}\right)\right) \times \partial / \partial X_{i}$ on an open set of $D \times \mathbb{A}^{n}$ defines a vector field $\tilde{\theta}$ on an open set of $D \times X_{\text {reg }} \subset D \times \mathbb{A}^{n}$, where $\left(X_{\mathrm{reg}} \subset\right) X \longrightarrow \mathbb{A}^{n}$ is defined by $x_{1}, x_{2}, \cdots, x_{n}$.

Therefore there exists an open set $U \subset D \times X_{\text {reg }}$ such that

(1.16) $0 \rightarrow O_{U} \theta \rightarrow T_{L^{r}}$ defines a regular system of Pfaffian differential equations of corank 1 on $U$.

Definition (1.17). We say that the system (1.16) of Pfaffian differential equations is defined by $\theta$ over $K$ or (1.16) is associated with $\theta$. We denote the system (1.16) by $\operatorname{Pf}(\theta)$. We do not make the open set $U$ precise and we say simply that $\operatorname{Pf}(\theta)$ is a system of Pfaffian differential equations on $D \times X$.

The dual form to (1.16) is given by the set of 1 -forms $d x_{i}-$ $\left(F_{i}\left(t ; x_{1}, x_{2}, \cdots, x_{n}\right)\right) /\left(G_{i}\left(t ; x_{1}, x_{2}, \cdots, x_{n}\right)\right) d t(1 \leqq i \leqq n)$ :

$$
0 \rightarrow \sum_{i=1}^{n} O_{U}\left(d x_{i}-\frac{F_{i}}{G_{i}} d t\right) \rightarrow \Omega_{U}^{1}
$$

Definition (1.18). If the exists a subfield $L \supset \mathbb{C}$ of $K$ and a derivation $\theta_{1} \in \operatorname{Der}_{L}\left(L\left(X \otimes_{\mathrm{C}} L\right), L\left(X_{\mathrm{C}} \otimes_{\mathbb{C}} L\right)\right)$ such that $\theta=\theta_{1} \otimes_{L} K$, then we say that the system $\operatorname{Pf}(\theta)$ of Pfaffian differential equations is defined over $L$ or the coefficients of the system $\operatorname{Pf}(\theta)$ is in $L$.

The most important case is the follows. Let $F: D \rightarrow \operatorname{Bim} X$ be a morphism of functors. Let $F$ be $K$-valued for a field $K$ of meromorphic functions such that $K$ is closed by differentiation. We put

$$
\Theta(F)(f(z))=\lim _{h \rightarrow 0} \frac{f\left(F(t+h) F(t)^{-1} z\right)}{h} \quad \text { for } f(z) \in \mathbb{C}(X) .
$$

By the same argument as in the case of algebraic operation, we can show $\theta(F)(f(z)) \in K\left(X \otimes_{\mathrm{C}} K\right)$ and $\Theta(F): \mathbb{C}(X) \rightarrow K\left(X \otimes_{\mathrm{C}} K\right)$ is a $\mathbb{C}$-derivation. Therefore we can extend $\theta(F)$ by sending $1 \otimes K$ to 0 to a $K$-derivation $K\left(X \otimes_{\mathrm{C}} K\right) \rightarrow K\left(X \otimes_{\mathrm{C}} K\right)$ which we denote also by $\Theta(F) . \quad \Theta(F)$ therefore 
defines a system of Pfaffian differential equations $\operatorname{Pf}(\Theta(F))$ on $X \times D$.

Definition (1.19). We call $\operatorname{Pf}(\Theta(F))$ the system of Pfaffian differential equations associated with the morphism $F: D \rightarrow \operatorname{Bim} X$ of functors. We denote it by $\operatorname{Pf}(F)$.

It follows from Lemma (1.27) Part I that if we replace $D$ by an appropriate subdomain $D^{\prime}$ there exists an algebraic variety $Z$, a morphism $f: Z \rightarrow \operatorname{Bir} X$ of functors and a holomorphic map $g: D^{\prime} \rightarrow Z^{\text {an }}$ such that the image $g\left(D^{\prime}\right)$ is dense in the algebraic variety $Z$ and

$$
f^{\text {an }} \circ g=F \text {. }
$$

The morphism $g: Z \rightarrow \operatorname{Bir} X$ is given by a family of birational automorphisms $\tilde{g}: Z \times X \mapsto Z \times X$ as in Lemma (1.9). Let $\psi: Z \times X \stackrel{\tilde{g}}{\rightarrow} Z \times X \stackrel{p_{2}}{\rightarrow} X$ be the composite rational map. Let us write $\psi(z, x)=z x$ for a general point $(z, x) \in Z \times X$. Therefore for general $t \in D^{\prime}$ and $x \in X, g(t) x$ is defined. We may write $g(t) x=F(t) x$. Then as in the case of algebraic operation, we can show that if we put for $x \in X, D^{\prime \prime}=\left\{t \in D^{\prime \prime} \mid \psi\right.$ is regular at $\left.(g(t), x)\right\}$, then $\left.D^{\prime \prime} \rightarrow D^{\prime \prime} \times X, t \mapsto(t, g(t) x)\right\}$ is a solution of the system $\operatorname{Pf}(F)$. If there exists a point $t_{0} \in D^{\prime}$ such that $F\left(t_{0}\right)=\operatorname{Id}_{X}$, let $X_{0}=\{x \in X \mid \psi$ is regular at $(g(t), x)\}$. Then $X_{0}$ is a non-empty Zariski open set of $X$ and any solution $t \mapsto(t, s(t)) \in D \times X$ of the system $\operatorname{Pf}(F)$ is given by the above form by the unicity of the solution at a regular point.

Remark (1.20). Let $K$ be a field of meromorphic functions on a domain $D$. Let $\theta \in \operatorname{Der}_{K}\left(K\left(X \otimes_{\mathrm{c}} K\right), K\left(X \otimes_{\mathrm{c}} K\right)\right)$. We consider the system $\operatorname{Pf}(\theta)$ of Pfaffian differential equations on $D \times X$. We are interested how we can construct the general solution of $\operatorname{Pf}(\theta)$ starting from $K$. Namely we are concerned with the extension $L$ over $K$ generated by the coordinates of the general solution $D^{\prime} \rightarrow D^{\prime} \times X, t \rightarrow(t, s(t)): L=K(\{s \circ \varphi \mid \varphi \in \mathbb{C}(X)$ (algebraic) rational function on $X\}$ ). Therefore if $D_{1} \subset D$ be a subdomain, we identify the systems on $D \times X$ and on $D_{1} \times X$ defined by $\theta$ since by the restriction the fields involved are isomorphic. As we are interested in the general solution, if $Y$ is birationally isomorphic to $X$, then $K\left(X \otimes_{\mathrm{c}} K\right) \cong K\left(Y \otimes_{\mathrm{c}} K\right)$ and by this isomorphism $\theta$ is considered as an element of $\operatorname{Der}_{K}\left(K\left(Y \otimes_{\mathrm{c}} K\right), K\left(Y \otimes_{\mathrm{c}} K\right)\right)$ and the general solutions on $X \times D^{\prime}$ and on $Y \times D^{\prime}$ are identified by the birational isomorphism between $X$ and $Y$. Therefore we identify also the system $\operatorname{Pf}(\theta)$ on $D^{\prime} \times X$ and the system on $D^{\prime} \times Y$. 
We need following

Lemma (1.21). Let $K$ be a field and $X, Y$ be absolutely irreducible algebraic varieties defined over $K$. If there exist a field extension $L \supset K$ such that $X \otimes_{K} L$ and $Y \otimes_{K} L$ are L-birationally isomorphic, then there exists a finite algebraic extension $M \supset K$ such that $X \otimes_{K} M$ and $Y \otimes_{K} M$ are $M$ birationally isomorphic.

Proof. Let $f: X \otimes_{K} L . \rightarrow Y \otimes_{K} L$ be $L$-birational isomorphism. Since $X$ and $Y$ are algebraic varieties, we may assume that $L$ is of finite type over $K$. Or there exist an integral domain $S=K\left[x_{1}, x_{2}, \cdots, x_{n}\right] \subset L$ such that the correspondence $\Gamma_{f} \subset(X \times Y) \otimes_{K} L$ is defined over Spec $S$ : namely there exists $\tilde{\Gamma} \subset(X \times Y) \otimes_{K}$ Spec $S$ such that $\Gamma_{f}=\tilde{\Gamma} \otimes_{S} L \subset\left((X \times Y) \otimes_{K}\right.$ Spec $\left.S\right)$ $\otimes_{S} L$. If we go to the algebraic closure $\bar{K}$, then $\tilde{\Gamma} \otimes_{K} \bar{K} \subset\left((X \times Y) \otimes_{K}\right.$ Spec $\left.S\right)$ $\otimes \bar{K}$ is a family of correspondences between $X \otimes_{K} \bar{K}$ and $Y \otimes_{K} \bar{K}$ parametrized by Spec $S \otimes_{K} \bar{K}$. Let $p: \tilde{\Gamma} \otimes_{K} \bar{K} \rightarrow X \times \operatorname{Spec} S \otimes_{K} \bar{K}$ (resp. $q: \tilde{\Gamma}$ $\left.\otimes_{K} \bar{K} \rightarrow Y \otimes \operatorname{Spec} S \otimes_{K} \bar{K}\right)$ be the restriction to $\tilde{\Gamma} \otimes_{K} \bar{K}$ of the projection

$$
\begin{aligned}
& \tilde{\Gamma} \otimes_{K} \bar{K} \subset X \times Y \times \text { Spec } S \otimes_{K} \bar{K}=\left(X \times \operatorname{Spec} S \otimes_{K} \bar{K}\right) \times \operatorname{Spec} \mathrm{S} \otimes_{K} \bar{K} \\
& \left(Y \times \operatorname{Spec} S \otimes_{K} \bar{K}\right) \rightarrow X \times \operatorname{Spec} S \otimes_{K} \bar{K}\left(\text { resp. } Y \times \text { Spec } S \otimes_{K} \bar{K}\right) .
\end{aligned}
$$

We have two natural $\bar{K}$-morphisms $\alpha: X \otimes_{K}$ Spec $S \otimes_{K} \bar{K} \rightarrow$ Spec $S \otimes_{K} \bar{K}$ and $\beta: \tilde{\Gamma} \otimes_{K} \bar{K} \rightarrow$ Spec $S \otimes_{K} \bar{K}$ so that the following diagram is commutative:

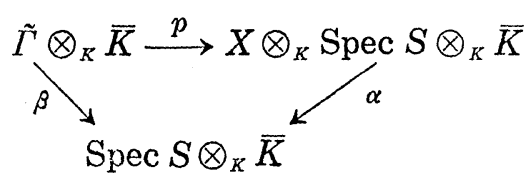

The morphisms $p$ and $q$ of algebraic varieties over $\bar{K}$ are birational. Therefore there exists a Zariski open sets $W, V$ of $\tilde{\Gamma} \otimes_{K} \bar{K}$ and of $X \times$ Spec $S \otimes_{K} \bar{K}$ isomorphic by $p$. It follows from (2.31) Proposition of [M] that there exists a Zariski open set $U$ of Spec $S \otimes_{K} \bar{K}$ such that there $\alpha(W)=\beta(V) \supset U$. For any closed point $s \in U, p \otimes \bar{K}(s)$ gives $\bar{K}$-birational morphism of $\tilde{\Gamma} \otimes_{K} \bar{K} \otimes_{\bar{K}} \bar{K}(s)$ to $X \times \operatorname{Spec} S \otimes_{K} \bar{K} \otimes_{\bar{K}} \bar{K}(s)=X \otimes_{K} \bar{K}$. By the same argument for $q$ we may assume that $q \otimes \bar{K}(s)$ gives $\bar{K}$-birational morphism of $\tilde{\Gamma} \otimes_{K} \bar{K} \otimes_{\bar{K}} \bar{K}(s)$ to $Y \rightarrow \operatorname{Spec} S \otimes_{K} \times \bar{K} \otimes_{\bar{K}} \bar{K}(s)=Y \otimes_{K} \bar{K}$ for any closed point $s \in U$. Therefore $X \otimes_{K} \bar{K}$ and $Y \otimes_{K} \bar{K}$ are $\bar{K}$-birationally equivalent. Since they are algebraic varieties, they are birationally isomorphic over a certain finite algebraic extension of $K$. 
Let $X$ be an algebraic variety and $G$ be an algebraic group over a field $K$. Let $\varphi: G \rightarrow \operatorname{Bir}(X)$ be a $K$-morphism of group functors. Then $\varphi$ induces a morphism $G(K[\varepsilon]) \rightarrow \operatorname{Bir} X(K[\varepsilon])$, where $K[\varepsilon]$ is the ring of dual numbers: $\varepsilon^{2}=0$. Let $K \rightarrow K[\varepsilon]$ be the canonical inclusion, then we get a commutative diagram

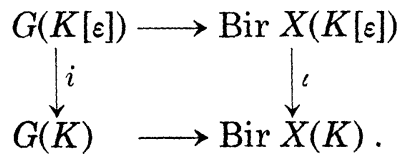

Therefore we get a map

$$
\varphi_{*}: \operatorname{Ker} i \longrightarrow \operatorname{Ker} \iota .
$$

Ker $i$ is the Lie algebra of $G$ (see Borel [B]) and Ker $\iota$ is identified with the Lie algebra of $K$-derivations of the function field $K(X)$ and $\varphi_{*}$ is a morphism of Lie algebras (see [D]).

Definition (1.22). Let $K$ be a field and $F\left(X_{1}, X_{2}, \cdots, X_{n}\right) \in K\left[X_{1}, X_{2}\right.$, $\cdots, X_{n}$ ]. We say that the polynomial $F$ is absolutely irreducible if for any extension $L \supset K, F$ is irreducible in the unique fectorization domain $L\left[X_{1}, X_{2}, \cdots, X_{n}\right]$. This is equivalent to saying that $V(F) \subset \mathbb{A}_{L}^{n}$ is irreducible.

It follows from Proposition (1.3) that to see whether $F$ is absolutely irreducible or not it is sufficient to check it for an algebraic closed extension of $K$.

Lemma (1.23). Let $L$ be a field (of characteristic 0) and $M \supset L$ be a field extension. Let $F(X), G(X) \in L\left[X_{1}, X_{2}, \cdots, X_{n}\right]$ be relatively prime in the unique factorization domain $(=U . F . D) L.\left[X_{1}, X_{2}, \cdots, X_{n}\right]$. Then $F(X)$ and $G(X)$ are relatively prime in $M\left[X_{1}, X_{2}, \cdots, X_{n}\right]$.

Proof. As we see below, it is sufficient to assume that $L$ is perfect. Assume that $F(X)$ and $G(X)$ are not relatively prime in $M\left[X_{1}, X_{2}, \cdots, X_{n}\right]$. There exists an $H(X) \in M\left[X_{1}, X_{2}, \cdots, X_{n}\right]$ which is not constant and divides $F(X)$ and $G(X)$. Geometrically $V(H) \subset V(F)$ and $V(H) \subset V(G)$ in $\mathbb{A}_{M}$. Therefore the zero locus $V(H)$ is a component of $V(F)$ and $V(G)$ on $\mathbb{A}_{M}$. Therefore the subvariety $V(H) \subset \mathbb{A}_{M}$ is defined over an algebraic closure $\bar{L}$ since $F, G \in L\left[X_{1}, X_{2}, \cdots, X_{n}\right]$. Or $F$ and $G$ have a non-trivial common factor in $\bar{L}\left[X_{1}, X_{2}, \cdots, X_{n}\right]$. Thus we may assume $H(X) \in \bar{L}\left[X_{1}, X_{2}, \cdots, X_{n}\right]$. Let $N \supset L$ be a finite Galois extension such that $H(X) \in N\left[X_{1}, X_{2}, \cdots, X_{n}\right]$ 
and $\mathscr{G}$ be the Galois group of $N / L$. The Galois group $\mathscr{G}$ operates on $N\left[X_{1}, X_{2}, \cdots, X_{n}\right]$ through the coefficients. Let $\mathscr{H}=\left\{g \in \mathscr{G} \mid H^{g}(X)=H(X)\right\}$. Since $V(F), V(G)$ in $\mathbb{A}_{N}^{n}$ are $G$-invariant, $V\left(H^{g}\right) \subset V(F), V(G)$ for any $g \in G$ : the Galois group $\mathscr{G}$ operates on $\mathbb{A}_{N}^{n}$ by $\left(x_{1}, x_{2}, \cdots, x_{n}\right) \mapsto\left(g x_{1}, g x_{2}, \cdots\right.$, $\left.g x_{n}\right)$ for $\left(x_{1}, x_{2}, \cdots, x_{n}\right) \in \mathbb{A}_{N}^{n}, g \in \mathscr{G}$. Therefore if we put $I(X)=\prod_{g \in \mathscr{g} / H} H^{g}(X)$, then $I(X) \in L\left[X_{1}, X_{2}, \cdots, X_{n}\right]$ and $V(I(X)) \subset V(F(X))$ and $V(I(X)) \subset V(G(X))$ on $\mathbb{A}_{N}^{n}, \quad V(F(x))$ and $V(G(X))$ have a common component in $\mathbb{A}_{N}^{n}$ and hence they are not relatively prime in $L\left[X_{1}, X_{2}, \cdots, X_{n}\right]$.

By Lemma (1.23) we can say that the polynomials $F$ and $G$ are relatively prime without mentioning the field of reference $M \supset L$.

Definition (1.24). Let $V$ be an algebraic variety defined over $k$. Let $f$ : Spec $k \rightarrow V$ be a $k$-valued point. Then $f \otimes_{k} K$ : Spec $K=\operatorname{Spec} k \times_{k}$ Spec $K \rightarrow V \otimes_{k} K$ is a $K$-valued point. We say that the $K$-valued point $f \otimes_{k} K$ is defined over $k$.

We need the following Proposition which comes from the fact that the complex number field $\mathbb{C}$ is algebraically closed and of infinite transcendence degree over any finitely generated subfield of $\mathbb{C}$.

Proposition (1.25). Let $V$ be an algebraic variety defined over $\mathbb{C}$. Let $F$ be an extension of $\mathbb{C}$. Then the set of points of $V \otimes_{\mathrm{C}} F$ defined over $\mathbb{C}$ is dense in $V \otimes_{\mathrm{C}} F$.

Proof. We may assume that $V$ is affine: $V \subset \mathbb{A}_{\mathrm{c}}^{n}$. Let $V \subset \mathbb{A}_{\mathrm{c}}^{n}$ is defined by the ideal $I \subset \mathbb{C}\left[X_{1}, X_{2}, \cdots, X_{n}\right]$ such that $V=\operatorname{Spec} \mathbb{C}\left[X_{1}, X_{2}, \cdots, X_{n}\right] / I$. We have to show the following. Let $A(X) \in F\left[X_{1}, X_{2}, \cdots, X_{n}\right]$ vanish on $\left\{\left(x_{1}, x_{2}, \cdots, x_{n}\right) \in \mathbb{C}^{n} \mid f\left(x_{1}, x_{2}, \cdots, x_{n}\right)=0\right.$ for all $\left.f \in I\right\}$. Then $A(X) \in F\left[X_{1}\right.$, $\left.X_{2}, \cdots, X_{n}\right] \cdot I$. Let $k \subset \mathbb{C}$ be a field finitely generated over $\mathbb{Q}$ such that $V \subset \mathbb{A}_{\mathbf{C}}^{n}$ is defined over $k$ : let $V_{0} \subset \mathbb{A}_{k}^{n}$ be defined by the ideal $I_{0} \subset k\left[X_{1}\right.$, $\left.X_{2}, \cdots, X_{n}\right]$ so that $I=\mathbb{C}\left[X_{1}, X_{2}, \cdots, X_{n}\right] I_{0}$. Let $F \supset L \supset k$ be a finite extension of $k$ such that $A(X) \in L\left[X_{1}, X_{2}, \cdots, X_{n}\right]$.

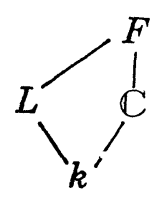

Since the degree of transcendence of $\mathbb{C}$ over $k$ is infinite and $\mathbb{C}$ is algebraically closed, we can find a point $\left(x_{1}, x_{2}, \cdots, x_{n}\right) \in \mathbb{C}^{n}$ with $f\left(x_{1}, x_{2}, \cdots, x_{n}\right)$ 
$=0$ for any $f \in I_{0}$ such that tr.d $L\left[X_{1}, X_{2}, \cdots, X_{n}\right] / L\left[X_{1}, X_{2}, \cdots, X_{n}\right] . I_{0}=$ tr.d $L\left[x_{1}, x_{2}, \cdots, x_{n}\right]$. We have an $L$-morphism of integral domains $\varphi$ : $L\left[X_{1}, X_{2}, \cdots, X_{n}\right] / L\left[X_{1}, X_{2}, \cdots, X_{n}\right] I_{0} \rightarrow L\left[x_{1}, x_{2}, \cdots, x_{n}\right]$. Since they have the same transcendence degree over $L, \varphi$ is an isomorphism. Since $A\left(x_{1}, x_{2}\right.$, $\left.\cdots, x_{n}\right)=0, A \in L\left[X_{1}, X_{2}, \cdots, X_{n}\right] I_{0} \subset F\left[X_{1}, X_{2}, \cdots, X_{n}\right] I$.

We use the following Lemma (1.26) in §4. The Lemma holds in far general setting and we need in fact the generalization. Since the generalization is proved by the same method, we give it in a special form.

Lemma (1.26). Let $K$ be a field containing $\mathbb{C}$ and $A_{i}\left(X_{1}, X_{2}\right), B_{i}\left(X_{1}, X_{2}\right)$ $\in K\left[X_{1}, X_{2}\right]$ for $i=1,2$ such that $\left(X_{1}, X_{2}\right) \rightarrow\left(A_{1} / B_{1}, A_{2} / B_{2}\right)$ defines an $K$ automorphism of the field $K\left(X_{1}, X_{2}\right)$. Moreover we assume that $A_{i}$ and $B_{i}$ are relatively prime. Let $B$ be the l.c.m. of $B_{1}$ and $B_{2}$ in $K\left[X_{1}, X_{2}\right]$. Then for a general $(a, b, c) \in \mathbb{C}^{3}$ the polynomial $C=a B+b B A_{1} / B_{1}+c B A_{2} / B_{2}$ is absolutely irreducible: for any field extension $L \supset K$ the polynomial $C \in$ $L\left[X_{1}, X_{2}\right]$ is irreducible.

Proof. Let $\bar{K}$ be an algebraic closure. The vector space $\bar{K} B_{1} B_{2}+$ $\bar{K} B A_{1} / B_{2}+K B A_{2} / B_{2} \subset \bar{K}\left(X_{1}, X_{2}\right)$ defines on $\mathbb{P}_{\bar{K}}^{2}$ a linear system $\mathscr{L}$ free from fixed components. The linear system $\mathscr{L}$ is not composite with a pencil. Therefore by the Theorem of Bertini (Zariski [Z], Theorem (I.6.3), p. 30). The general cycle is absolutely irreducible. Namely let $u_{0}, u_{1}, u_{2}$ be variables over $\bar{K}$. Then the polynomial $u_{0} B+U_{1} B A_{1} / B_{1}+u_{2} B A_{2} / B_{2}$ with coefficients in $\overline{K\left(u_{0}, u_{1}, u_{2}\right)}$ is absolutely irreducible. Geometrically, if we consider an $\bar{K}$-algebraic variety $W_{0}=\left\{\left(a_{0}, a_{1}, a_{2} ; b_{1}, b_{2}\right) \in A_{R}^{3} \times A_{R}^{2}\right\}$ $\left.\left(a_{0} B+a_{1} B A_{1} / B_{1}+a_{2} B A_{2} / B_{2}\right)\left(b_{1}, b_{2}\right)=0\right\}, W_{0}$ is naturally a subset of $A_{R}^{3} \times$ $\mathbb{P}_{\bar{R}}^{2}$. We denote by $W$ the closure $\bar{W}_{0} \subset A_{R}^{3} \times \mathbb{P}_{R}^{2}$. The projection $A_{R}^{3} \times \mathbb{P}_{R}^{2}$ $\rightarrow \bar{W}_{0}$ induces $p: W \rightarrow \mathbb{A}_{R}^{3}$. The theorem of Bertini says that the generic fibre of $P$ is reduced and absolutely irreducible (géométriquement intègre, in the language of E.G.A. Chap IV). Since $p$ is generically flat by E.G.A. Chap IV, §6, Théorème (6.9.1), Lemma follows from Proposition (1.25) and from E.G.A. Chap IV, §12, Théorème (12.2.4).

\section{§ 2. Permissible operations}

We treat sets of meromorphic functions. Very often they are rings or fields. We have to clarify several points. First of all, we identify a holomorphic function $f$ on an open set $U \subset \mathbb{C}$ with its restriction $f \mid V$ to an open subset $V \subset U$. We shall consider only the following type of sets, 
rings or fields of meromorphic functions if we do not make any particular assumption: (2.1) a set, a ring or a field of meromorphic functions on a domain $D$. The ring or field structure is defined by the usual addition and product of meromorphic functions.

We consider algebraic varieties defined over a ring $R$ or a field $K$ of meromorphic functions and morphisms between them. In this case, we can replace $R, K$ respectively by a ring or field of meromorphic functions of finite type over $\mathbb{C}$. Therefore in the following discussion, we often assume without making it precise that there exists a domain $D$ such that the ring $R$ consists of holomorphic functions on $D$ and the field $K$ is the quotient field of such a ring.

Now given a set $S$ of meromorphic functions on a domain $D \subset \mathbb{C}$, what operations are permissible to construct new functions from $S$ ? In other words if we call the set $S$ the set of the known functions, what are newly known functions from the set $S$ by a reasonable operations?

The operations allowed by Painlevé in his famous Stockholm Lessons [P] are as follows.

(O) Let $f(z) \in S$. Then the derived function $f^{\prime}(z)$ is a new known functions.

This operation (O) is of a special nature as we see later. This is the golden rule which we can not touch.

(P1) If $f_{1}, f_{2} \in S$, then the sum $f_{1}+f_{2}$ and the product $f_{1} f_{2}$ are new known functions. Moreover if $f_{2} \neq 0$, then the quotient $f_{1} / f_{2}$ is a new known function too.

(P2) Let $a_{1}, a_{2}, \cdots, a_{n} \in S$. Then an algebroid function $f$ or any solution of an algebraic equation $f^{n}+a_{1} f^{n-1}+\cdots+a_{n}=0$, is a new known function. Notice that here we consider $f$ as a single valued function on an appropriate smaller domain.

(P3) Let $f(z) \in S$. Then the quadrature $\int f(z) d z$ is a new known function.

(P4) Let $a_{1}, a_{2}, \cdots, a_{n} \in S$. Then any solution $f$ of the linear differential equation $d^{n} f / d z^{n}+a_{1}\left(d^{n-1} f / d z^{n-1}\right)+a_{2}\left(d^{n-2} f / d z^{n-2}\right)+\cdots+a_{n+1} f=0$ is a new known function.

(P5) Let $\Gamma \subset \mathbb{C}^{n}$ be a lattice such that the quotient $\mathbb{C}^{n} / \Gamma$ is an abelian variety or an algebraic torus. Let $\pi: \mathbb{C}^{n} \rightarrow \mathbb{C}^{n} / \Gamma$ be the projection. Let $f_{1}, f_{2}, \cdots, f_{n} \in S$ be regular on a domain $D$ and $\varphi$ be a meromorphic function on $\mathbb{C}^{n} / \Gamma$. Then the function $\varphi \circ \pi \circ\left(f_{1}, f_{2}, \cdots, f_{n}\right)$ is a new known 
function if it is not the constant function taking $\infty$.

$$
\begin{aligned}
& D \rightarrow \mathbb{C}^{n} \stackrel{\pi}{\longrightarrow} \mathbb{C}^{n} / \Gamma \cdots \stackrel{\varphi}{ } \stackrel{\mathbb{C}}{ } \\
& a \mapsto\left(f_{1}(z), f_{2}(z), \cdots, f_{n}(z)\right)
\end{aligned}
$$

In all the operations (P2), (P3), (P4) and (P5), we have to choose an appropriate subdomain $D^{\prime}$ such that the solutions are meromorphic and single valued on $D^{\prime}$.

Remark (2.2). In the operation (P5), we had to avoid meromorphic functions $\varphi \circ \pi$ such that the pole of the meromorphic function $\varphi \circ \pi$ contains the image of $D$. Similar cases happen in what follows but we do not repeat this remark.

Given a set $S$ of meromorphic functions on a domain $D$ and an algebraic differential equation $E=0$ with coefficients in $S$, the problem of integration of the differential equation $E=0$ is to know whether starting from the set $S$ and by iterating finite number of times of the permissible operations (O), (P1), (P2), . , (P5), we can express the solutions of the differential equation $E=0$.

LEMMA (2.3). If we admit the operations (O) and (P1) and if $S$ contains all the constant functions, the permissible operation $(\mathrm{P} 4)$ is equivalent to

(P4)' Let $a_{i j}(z) \in S$ be holomorphic functions $(1 \leqq i, j \leqq n)$. Then any solutions $f_{1}(z), f_{2}(z), \cdots, f_{n}(z)$ of the lienar differential equations

$$
\left(\begin{array}{c}
f_{1}^{\prime}(z) \\
f_{2}^{\prime}(z) \\
\vdots \\
f_{n}^{\prime}(z)
\end{array}\right)=\left(a_{i j}(z)\right)\left(\begin{array}{c}
f_{1} \\
f_{2} \\
\vdots \\
f_{n}
\end{array}\right)
$$

are new known functions.

Proof. In fact, assume $a_{1}, a_{2}, \cdots, a_{n} \in S$ be given. The solution $f(z)$ of the differential equation

$$
\frac{d^{n} f}{d z^{n}}+a_{1} \frac{d^{n-1} f}{d z^{n-1}}+\cdots+a_{n} f=0
$$

satisfies

$$
\left\{\begin{array}{l}
f_{1}^{\prime}(z)=f_{2}(z) \\
f_{2}^{\prime}(z)=f_{3}(z) \\
\vdots \\
f_{n-1}^{\prime}(z)=-\left(a_{1} f_{n-2}+a_{2} f_{n-3}+\cdots+a_{n} f_{1}\right)
\end{array}\right.
$$


if we put $f_{1}(z)=f(z)$. Therefore (P4) is a special case of $\left(\mathrm{P} 4^{\prime}\right)$. We did not use the operations $(\mathrm{O})$ and $(\mathrm{P} 1)$. Conversely let $a_{i j} \in S$ be given $(1 \leqq i, j \leqq n)$ and let $f_{1}, f_{2}, \cdots, f_{n}$ be solutions of the differential equation

$$
\left(\begin{array}{c}
f_{1}^{\prime}(z) \\
f_{2}(z) \\
\vdots \\
f_{n}^{\prime}(z)
\end{array}\right)=\left(a_{i j}(z)\right)\left(\begin{array}{c}
f_{1}(z) \\
f_{2}(z) \\
\vdots \\
f_{n}(z)
\end{array}\right) .
$$

Let $K=\mathbb{C}\left(a_{i j}(z), a_{i j}^{\prime}(z), a_{i j}^{\prime \prime}(z), \cdots\right)_{1 \leqq i, j \leqq n}$ be the field of rational functions in $a_{i j}$ 's and their derivatives. Its sufficient to show that for any $1 \leqq i \leqq n$, there are $a_{l}(1 \leqq l \leqq m)$ in $K$ such that $f_{i}$ satisfies the differential equation

$$
\frac{d^{m} f_{i}}{d z^{m}}+a_{1} \frac{d^{m-1} f_{i}}{d z^{m-1}}+\cdots+a_{m} f_{i}=0
$$

since by the permissible operations (O) and (P1) and by the hypothesis $S \supset \mathbb{C}$, all the functions in $K$ hence in particular $a_{l}$ 's are known functions. In fact $d f_{i} / d z$ is a linear combinations of the $f_{j}$ with coefficients in $\mathbb{C}\left(a_{i j}\right)_{1 \leqq i, j \leqq n}$. By induction and (2.3.1), $d^{k} f_{i} / d z^{k}$ is a linear combination of the $f_{j}$ with coefficients in $K$. Since there are $n f_{j}$ 's there is a non-trivial $K$-linear relations among $(n+1)$-elements $d^{k} f_{i} / d z^{k}(0 \leqq k \leqq n)$,

$$
c_{0} \frac{d^{n} f_{i}}{d z^{n}}+c_{1} \frac{d^{n-1} f_{i-1}}{d z^{n-1}}+\cdots+c_{n} f_{i}=0,
$$

which gives (2.3.2).

The first question that one might have is the following. Are these operations reasonable? Or in other words, does the rule of the game above have mathematically a sense? This question is illustrated by the resolution of algebraic equation by radicals. In the resolution of algebraic equation, historically or by experience the permissible operations are the addition. the multiplication, the division in fields and the extraction of radicals. The Galois theory tells us that in fact allowing these operations is equivalent to allowing successive cyclic extensions and hence nicely formulated by the group theory.

As we see below, Painlevé's operations (P1), (P2), .., (P5) are quite group theoretic too, despite of their appearance. These operations (P1), (P2), .., (P5) are not only easy operations come into our mind one by one but also they are related with algebraic groups.

To explain our interpretation of the permissible operations, we need 
some preliminaries.

Let $G$ be a (connected) algebraic group defined over $\mathbb{C}$ and we denote by $g$ its Lie algebra. The tangent bundle $T_{G}$ of $G$ is trivial and we trivialized it by using right invariant vector fields on $G$. The trivialization $G \times \mathfrak{g} \cong T(G)$ is done algebraically and canonically. Let $F: D \rightarrow G$ be a holomorphic map of a domain $D$ of $\mathbb{C}$ to $G$ (more precisely to the associate analytic space $\left.G^{\text {an }}\right)$. The curve $F$ defines a holomorphic vector field $X$ on $F(D)$. Namely

$$
X_{F(x)}(f)=\lim _{h \rightarrow 0} \frac{f(F(x+h))-f(F(x))}{h}
$$

for $x \in D$ and for a holomorphic function $f$ around $F(x)$. We have thus a holomorphic map $\tilde{F}: D \rightarrow T(G) \cong G \times \mathfrak{g}$. Composing with the projection $p_{2} G \times \mathfrak{g} \rightarrow \mathfrak{g}$, we get a holomorphic map $p_{2} \circ \tilde{F}: D \rightarrow \mathfrak{g}$ which we denote by $F^{\prime}$ or by $\partial F$.

Definition (2.4). We say that $F^{\prime}$ is the derivative of the curve $F$ and $F$ is the integral of $F^{\prime}$.

ExAmple (2.5). (1) The simplest case is the additive group $G=\mathbb{G}_{a}$ (= C). If we trivialize $T\left(\mathbb{G}_{a}\right)$ by using the invariant vector field $d / d x$ with $\mathbb{G}_{a}=\operatorname{Spec} \mathbb{C}[x]$, when the function $F: D \rightarrow G=\mathbb{C}$ is given, $F^{\prime}: D \rightarrow \mathbb{C}$ is $d F / d z$ where $z$ is the usual coordinate of $D \subset \mathbb{C}$.

(2) Let us study the case of the multiplicative group $G=\mathbb{G}_{m}\left(=\mathbb{C}^{*}\right)$. Let us trivialize $T\left(\mathbb{G}^{m}\right)$ by using the invariant vector field $t(d / d t)$ with $\mathbb{G}_{m}=\operatorname{Spec} \mathbb{C}\left[t, t^{-1}\right]$. Let $F: D \rightarrow \mathbb{G}_{m} \subset \mathbb{C}$ be a holomorphic function. Then $F^{\prime}: D \rightarrow T(\mathbb{C})$ is given by $z \mapsto F^{\prime}(z)(d / d t)_{F(z)}$. To identify $T\left(\mathbb{G}_{m}\right)$ with $\mathbb{G}_{m}$ $\times \mathbb{C}$ by using $t(d / d t)$ is to express vector fields by using the translation invariant base $t(d / d t)$. Hence $\tilde{F}: D \rightarrow \mathbb{G}_{m} \times g$ is $z \mapsto\left(F(z),\left(F^{\prime}(z) / F(z)\right)\right.$ $\left.\times(t(d / d t))_{F(z)}\right)$ and $\partial F: D \rightarrow \mathbb{C}$ is $z \mapsto F^{\prime}(z) / F(z)$.

(3) More generally let us study the case $G=G L_{n}(\mathbb{C})$. Its Lie algebra $\mathrm{gl}_{n}$ is the Lie algebra of all the $n \times n$-matrices. Let $F: D \rightarrow G L_{n}(\mathbb{C})$ be given by $F(z)=\left(f_{i j}(z)\right)$. We use as a basis of right invariant vector fields $X_{i j}(g)$ $=R_{g^{*}}\left(\left(\partial / \partial x_{i j}\right)_{I_{n}}\right)$ for $g \in G L_{n}(\mathbb{C})$, the translation of $\partial / \partial x_{i j}$ at $I_{n}$, where $x_{i j}$ are the natural coordinate system on $G L_{n}(\mathbb{C}) \subset M_{n}(\mathbb{C})$ and $R_{g}: G L_{n}(\mathbb{C}) \rightarrow$ $G L_{n}(\mathbb{C})$ is the multiplication from the right by $g . F: D \rightarrow T\left(G L_{n}(\mathbb{C})\right) \subset$ $T\left(M_{n}(\mathbb{C})\right)$ is given by $z \mapsto \sum_{i j} f_{i j}^{\prime}(z)\left(\partial / \partial x_{i j}\right) f(z)$. As in example (2), we have to look for $a_{i j}(g)$ such that 


$$
\sum_{i, j} f_{i j}^{\prime}(z)\left(\frac{\partial}{\partial x_{i j}}\right)_{F(z)}=\sum_{i, j} a_{i j}(F(z)) R_{g *}\left(\left(\frac{\partial}{\partial x_{i j}}\right)_{I_{n}}\right) \text {, with } g=F(z) .
$$

Let us calculate $R_{g *}\left(\left(\partial / \partial x_{i j}\right)_{I_{n}}\right)$ for $g=\left(g_{k l}\right) \in G L_{n}(\mathbb{C})$.

$$
\begin{gathered}
R_{g^{*}}\left(\left(\frac{\partial}{\partial x_{i j}}\right)_{I_{n}}\right)\left(x_{l m}\right)=\frac{\partial}{\partial x_{i j}}\left(x_{l m} \circ R_{g}\right) \\
=\frac{\partial}{\partial x_{i j}}\left(\sum_{k} x_{l k} g_{k m}\right)=\delta_{i l} g_{j m} .
\end{gathered}
$$

Hence

$$
\begin{gathered}
\sum_{l, j} a_{i j}(F(z)) R_{g^{*}}\left(\left(\frac{\partial}{\partial x_{i j}}\right)_{I_{n}}\right)\left(x_{l m}\right) \\
=\sum_{i, j} a_{i j}(F(z)) \delta_{i l} g_{j m} \\
=\sum_{j} a_{l j}(F(z)) g_{j m} .
\end{gathered}
$$

Therefore $\left(f_{i j}^{\prime}(z)\right)=\left(a_{i j}(F(z))\right) F(z)$.

Thus $F^{\prime}: D \rightarrow \mathfrak{g l}_{n}$ is given by

$$
F^{\prime}(z)=\left(a_{i j}(F(z))\right)=\left(f_{i j}^{\prime}(z)\right) F(z)^{-1} .
$$

(4) The case where $G$ is an abelian variety is simple. Let $p: \mathbb{C}^{n} \rightarrow G$ the universal covering of $G$ so that there exists a lattice $L \subset \mathbb{C}^{n}$ with $\mathbb{C}^{n} / L \cong G$. Assume that we can lift $F$ to $\bar{F}: D \rightarrow \mathbb{C}^{n}, \bar{F}$ is given by $\left(f_{1}(z), \cdots, f_{n}(z)\right)$ and hence $\tilde{\bar{F}}^{\prime} \rightarrow T\left(\mathbb{C}^{n}\right)$ is given by $\sum_{i=1}^{n} f_{i}^{\prime}(z)\left(\partial / \partial z_{i}\right)$, if we take a usual coordinate system $\left(z_{1}, z_{2}, \cdots, z_{n}\right)$ of $\mathbb{C}_{n}$ and we use $\partial / \partial z_{1}, \partial / \partial z_{2}$, $\cdots, \partial / \partial z_{n}$ as a basis of translation invariant vector fields on $\mathbb{C}^{n}$ hence on $G$. Therefore the map $F^{\prime}: D \rightarrow \mathrm{g} \cong \mathbb{C}^{n}$ is given by $z \mapsto\left(f_{1}^{\prime}(z), f_{2}^{\prime}(z), \cdots, f_{n}^{\prime}(z)\right)$.

The derivative of a curve has another interpretation from the algebraic geometry. Let $U$ be an affine open neighbourhood of the unit element 1 of the algebraic group $G$. Let $R(D)$ be the $\mathbb{C}$-algebra of all the holomorphic functions on $D$ and $K(D)$ the field of all the meromorphic functions on $D$.

The group $G$ operates on $G$ by the left multiplication which we denote by $(G, G)$. Then the curve $F$ defines the vector field $\Theta(F,(G, G))$ on the $K(D)$-algebraic variety $G \otimes_{\mathrm{c}} K(D)$ by (1.14). We put $\tau(F)=\Theta(F$, $(G, G))(1)$ which is the value of the vector field $\Theta(F,(G, G))$ on $G \otimes_{\mathrm{c}} K(D)$ at the unit element 1. Namely $\tau(F)$ is an element of $\mathfrak{g} \otimes_{\mathrm{c}} K(D)$.

On the other hand the derivative $F^{\prime}: D \rightarrow \mathfrak{g}$ can be regarded as an element of $\mathfrak{g} \otimes K(D)$. In fact, taking a basis $\left\{X_{1}, X_{2}, \cdots, X_{d}\right\}$ of $\mathbb{C}$-vector 
space $\mathfrak{g}$, we get an isomorphism $\mathfrak{g} \cong \mathbb{C}^{d}$ and by this isomorphism $F^{\prime}$ is a holomorphic map $\left(f_{1}, f_{2}, \cdots, f_{d}\right): D \rightarrow \mathbb{C}^{d}$. Then we consider $\sum_{i=1}^{d} X_{i} \otimes f_{i} \in$ $\mathrm{g} \otimes_{\mathbf{c}} K(D)$. It is easy to see $\sum_{i=1}^{d} X_{i} \otimes f_{i}$ is independent of the choice of basis of $\mathfrak{g}$.

Proposition (2.6) With the above canonical identification, we get $F^{\prime}=\tau(F)$.

Proof. It follows from the definition that

$$
X_{F(z)}=\sum_{i=1}^{d} f_{i}(z) R_{F^{\prime}(z) *} X_{i} \quad \text { for any fixed } z \in D .
$$

Since the $R_{g^{*}} X_{i}, g \in G$ from a basis of the right invariant vector fields on $G$, where $R_{g}(g \in G)$ denotes the multiplication by $g$ from the right. It follows from (2.6.1),

$$
R_{F^{(z)}-1 *} X_{F}=\sum_{i=1}^{l} f_{i}(z) X_{i} \quad \text { if we fix } z \in D .
$$

Let $f$ be a regular (rational) function in a (Zariski) open neighbourhood of 1. We operate the derivations in (2.6.2) to $f$. The left hand side is

$$
R_{F(z)-1 *} X_{F}(f)=X_{F}\left(R_{F(z)-1}^{*} f\right)=\lim _{h \rightarrow 0} \frac{f\left(F(z+h) F(z)^{-1}\right)-f(1)}{h},
$$

for $z \in D$. Therefore $R_{F^{(z)-1 *}} X_{F}$ is nothing but $\theta(F)(1)$ if we consider $z$ as a variable. Furthermore if we vary $z, \sum_{i=1}^{d} f_{i}(z) X_{i}$ becomes $\sum_{i=1}^{d} f_{i}(z) \otimes X_{i}$ and the Proposition is proved.

Remark (2.7). If we consider the Lie algebra $\mathfrak{g} \cong \mathbb{C}^{n}$ as an algebraic variety or if we consider $Y=\operatorname{Spec} S(\mathfrak{g})$, where $S(\mathfrak{g})$ denotes the symmetric algebra on the vector space $\mathfrak{g}$, the above element $\tau(F)$ in $\mathrm{g} \otimes K(D)$ identified with $F^{\prime}: D \rightarrow \mathrm{g}$ is nothing but $K(D)$-valued point $P\left(F^{\prime}\right) \in Y$ associated with $F^{\prime}: D \rightarrow Y^{\text {an }}=\mathfrak{g} \cong \mathbb{C}^{n}$; we had better consider the closed point $P\left(F^{\prime}\right) \in Y \otimes_{\mathrm{c}} K(D)=\mathfrak{g} \otimes_{\mathrm{c}} K(D)$.

In the solution of differential equations it is important to find a small subfield $L$ of $K(D)$ such that $\tau(F) \in \mathrm{g} \otimes_{\mathrm{c}} L$. More precisely, recalling that we identify a holomorphic function with its restriction on a subdomain, we adopt the following

Definition (2.8). Let $D^{\prime} \subset D$ be a subdomain of $D$ and $L$ be a subfield of the field $K\left(D^{\prime}\right)$ of the meromorphic functions on $D^{\prime}$. If there 
exists an element $\theta \in \mathfrak{g} \otimes_{\mathrm{c}} L$ such that $\tau(F)=\theta$ in $\mathrm{g} \otimes_{\mathrm{c}} K\left(D^{\prime}\right)$, we say that $\tau(F)$ is $L$-valued or $F^{\prime}: D^{\prime} \rightarrow \mathfrak{g}$ is defined over $L$. Here we consider $\mathfrak{g} \otimes_{\mathrm{c}} L \subset \mathfrak{g} \otimes_{\mathrm{c}} K\left(D^{\prime}\right), \mathfrak{g} \otimes_{\mathrm{c}} K(D) \subset \mathfrak{g} \otimes K\left(D^{\prime}\right)$ by the canonical inclusions $L \subset K\left(D^{\prime}\right), K(D) \subset K\left(D^{\prime}\right)$.

Let $K$ be a field of meromorphic functions on $D$ closed under the derivation. Let $F: D \rightarrow G$ be a $K$-rational point. Then it follows from $\S 1$ that $\partial F$ is $K$-valued.

Proposition (2.9). Let $\varphi: G_{1} \rightarrow G_{2}$ be a morphism of algebraic groups defined over $\mathbb{C}$ and $F: D \rightarrow G_{1}$ be a holomorphic curve. Let $\varphi_{*}: \mathfrak{g}_{1} \rightarrow \mathfrak{g}_{2}$ be the morphism of Lie algebras induced by $\varphi$. Then we have $\tau(\varphi \circ F)=$ $\varphi_{*} \otimes_{\mathrm{c}} K(D)(\tau(F))$, where $\varphi_{*} \otimes_{\mathrm{c}} K(D): \mathfrak{g}_{1} \otimes_{\mathrm{c}} K(D) \rightarrow \mathrm{g}_{2} \otimes_{\mathrm{c}} K(D)$ denotes the scalor extension of $\varphi_{*}$.

Proof. This is a direct consequence of the definition of $\tau$.

We can state Proposition in terms of derivatives.

Corollary $(2.10) . \quad(\varphi \circ F)^{\prime}=\varphi_{*} \circ F^{\prime}$.

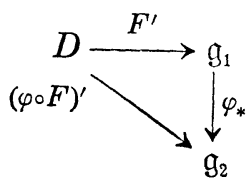

Corollary (2.11). Let $\mathbb{C} \subset K \subset K(D)$ be a subfield containing $\mathbb{C}$. If $\tau(F) \in \mathfrak{g}_{1} \otimes_{\mathrm{C}} K(D)$ is contained in $\mathfrak{g}_{1} \otimes_{\mathrm{C}} K$, then $\tau\left(\varphi_{1} \circ F\right)$ is also in $\mathfrak{g}_{2} \otimes_{\mathrm{C}} K$.

Proof. This is an immediate consequence of the Proposition.

The following Lemma is also a direct consequence of the definition.

Lemma (2.12). Let $F: D \rightarrow G$ be an analytic curve and $\mathbb{C} \subset K \subset K(D)$ be a subfield of $K(D)$ containing $\mathbb{C}$ such that $\tau(F) \in \mathfrak{d}_{1} \otimes_{\mathbb{C}} K$. Let $g \in$ $G \otimes_{\mathrm{C}} K(D)$ be defined over $K$. If we denote the adjoint representation $G \otimes_{\mathrm{C}} K(D) \rightarrow G L\left(\mathfrak{g} \otimes_{\mathrm{C}} K(D)\right)$ by ad, then $(\operatorname{adg})(\tau(F))$ is in $\mathfrak{g}_{1} \otimes_{\mathrm{C}} K$.

Proof. The adjoint representation is defined over $\mathbb{C}$ hence over $K$.

In the sequel it is important to choose a small subfield $K$ such that $\tau(F) \in \mathfrak{g}_{1} \otimes_{\mathrm{C}} K$.

Lemma (2.13). Let $F: D \rightarrow G$ be an analytic curve. Let $\mathbb{C} \subset K \subset K(D)$ be a subfield containing $\mathbb{C}$. Then the following conditions are equivalent.

(1) $\tau(F) \in \mathfrak{g}_{1} \otimes_{\mathrm{C}} K$. 
(2) For any rational function $f$ on $G$ regular at 1 , the derivation $\tau(F): O_{G \otimes \mathrm{C} K(D), 1} \rightarrow K(D)$ maps $f \in O_{G \otimes \mathfrak{C} K(D), 1}$ in $K$.

(3) There exists a basis $\left\{X_{1}, X_{2}, \cdots, X_{d}\right\}$ of $g$ such that

$$
F^{\prime}(z)=\sum_{i=1}^{d} f_{i}(z) X_{i} \quad \text { with } f_{i}(z) \in K
$$

(4) For any basis $\left\{X_{1}, X_{2}, \cdots, X_{d}\right\}$ of $\mathfrak{g}$ if

$$
F^{\prime}(z)=\sum_{i=1}^{d} f_{i}(z) X_{i}, \quad \text { then } f_{i}(z) \in K
$$

Proof. The equivalence of (1), (3) and (4) follows from the proof of Proposition (2.6). The equivalence of (1) and (2) is deduced from the definition.

Lemma (2.14). Let $G$ be an algebraic group defined over $\mathbb{C}, \mathfrak{g}$ its Lie algebra and $D \subset \mathbb{C}$ a domain. Let $f: D \rightarrow g$ be a holomorphic map. Then there exists a curve $F: D \rightarrow G$ such that $f$ is the derivative of $F$. Let $H: D \rightarrow G$ be another curve such that $H^{\prime}=f$. Then there exists an element $g \in G$ such that $H=R_{g} \circ F$, where $R_{g}: G \rightarrow G$ is the right multiplication of $g$.

Proof. Let us fix a point $x_{0} \in D$. Let us look for $F: D \rightarrow G$ such that (a) $F\left(x_{0}\right)=1 \in G$ and (b) $F^{\prime}=f$. Then the condition (b) is stated by differential equations $\varphi_{i}^{\prime}(z)=\Phi_{i}\left(\varphi_{1}(z), \varphi_{2}(z), \cdots, \varphi_{n}(z)\right)(1 \leqq i \leqq n)$ and (a) is equivalent to giving the initial conditions $\varphi_{i}\left(x_{0}\right)=11 \leqq i \leqq n$. This shows the existence. As question is local, let us take a local coordinate around $1 \in G$. Now let $g \in G$. Then $R_{g} \circ F$ is an integral of $f$. In fact

$$
X_{R_{g^{\circ} F^{\prime}(x)}}(\varphi)=\lim _{h \rightarrow 0} \frac{\varphi\left(R_{g} \circ F(x+h)\right)-\varphi\left(R_{g} \circ F(x)\right)}{h}=R_{g^{*}} X_{F^{\prime}(x)}(\varphi) .
$$

Let $D_{1}, D_{2}, \cdots, D_{n}$ be right invariant vector fields on $G$ and $X_{F(x)}=$ $\sum_{i=1}^{n} a_{i}(x) D_{i}$. Then

$$
R_{g^{*}} X_{F^{\prime}(x)}=R_{g^{*}} \sum_{i=1}^{n} a_{i}(x) D_{i}=\sum_{i=1}^{n} a_{i}(x) R_{g^{*}} D_{i}=\sum_{i=1}^{n} a_{i}(x) D_{i} .
$$

Hence $R_{g} \circ F$ is an integral of $f$ for any $g \in G$. Let now $H$ be another integral of $f$. Let $g=H\left(x_{0}\right)$. Then $H\left(x_{0}\right)=R_{g} \circ F$ by the uniqueness of the solution of a differential equations satisfying the same initial condition. 
We want to introduce an operation $Q$ and shall show that the operations (P1), (P2), $\cdots$, , (P5) are equivalent to the simple operation $Q$.

(Q) Let $G$ be an algebraic group of dimension $n$ defined over $\mathbb{C}$ and $g$ be its Lie algebra. Let $f_{1}, f_{2}, \cdots, f_{n} \in S$ be holomorphic over a domain $D$. Choose and fix a isomorphism $\mathfrak{g} \cong \mathbb{C}^{n}$ of vector space and let $f: D \rightarrow$ $\mathbb{C}^{n} \cong \mathfrak{g}$ be a holomorphic map. By Lemma (2.14) there exists an integral $F$ of $f$. For a rational function $\varphi$ on $G$, we get a new function $\varphi \circ F: D$ $\rightarrow G \cdots \rightarrow \mathbb{C}$.

Starting from $f_{1}, f_{2}, \cdots, f_{n}$ and an algebraic group $G$, we get a set of meromorphic functions $\{\varphi \circ F \mid \varphi \in k(G)\}$. Notice that the set of meromorphic functions $\{\varphi \circ F \mid \varphi \in k(G)\}$ is independent of the choice of the integral $F$ by Lemma (2.14). We set $Q\left(f_{1}, f_{2}, \cdots, f_{n} ; G\right)(S)=Q(f ; G)(S)=\{\varphi \circ F \mid \varphi \in k(G)\}$ or we denote it simply by $Q(S)$. We say that an element of $Q(f ; G)(S)$ obtained from $S$ by the operation $Q$ with respect to $f$ and $G$.

We can formulate the operation $Q$ in a completely algebro-geometric language.

Definition (2.15). Let $K \supset \mathbb{C}$ be a field of meromorphic functions on $D$ closed under the differentiation and $P$ : Spec $K \rightarrow G$ be a $K$-valued point of an algebraic group over $\mathbb{C}$. $P$ define a holomorphic curve $F: D^{\prime} \rightarrow G^{\text {an }}$ such that $P(F)=P$. Now as we have seen above $F$ gives $\partial F \in \mathfrak{g} \otimes_{\mathrm{c}} K$ where $g$ is the Lie algebra of $G$. Since $\partial F$ is determined by $P$ : Spec $K \rightarrow$ $G$, we denote it by $\partial P$.

Let $S$ be a set of known meromorphic functions on a domain $D$. Let $K$ be the field of meromorphic functions generated over $\mathbb{C}$ by the set $S$. Let $L \supset \mathbb{C}$ be a field of meromorphic functions on a subdomain of $D$. Then the field $L$ consists of new known functions from $S$ by the operation $Q$ if there exist a $L$-valued point $P: \operatorname{Spec} L \rightarrow G$ of an algebraic group over $\mathbb{C}$ such that (1) $P$ induces an isomorphism between the residue field $\mathbb{C}(P(\operatorname{Spec} L))$ and the field $L$, and such that (2) $\partial P$ is $K$-valued.

In fact, let $F: D^{\prime} \rightarrow G$ be a holomorphic curve such that $P(F)=P$. By the hypothesis (2) $\partial F: D^{\prime} \rightarrow \mathfrak{g}$ is defined over $K$. Then $L$ coincides with $K(\{\varphi \circ F \mid \varphi \in \mathbb{C}(X)\})$ by (1).

Definition (2.16). We denote by $S P$ (resp. $S Q$ ) the set of meromorphic functions obtained from $S$ by finite iterations of the permissible operations (O), (P1), ․, (P5) (resp. (O) and (Q)). In what follows, we always mean by an iteration of the operations a finite iteration. 
Remark (2.17). The sets $S P$ and $S Q$ do not satisfy the condition (2.1). But a subset of $S P$ (resp. $S Q$ ) obtained by a finite iteration of the operations (O), (P1), ․, (P5) (resp. (O), (Q)) satisfies the condition (2.1).

Remark (2.18). The operation $Q$ was considered by Kolchin [Ko], Chap IV from a different view point. Let $K$ be a differential field of meromorphic functions. In his language the differential field $L=K\langle K Q(f ; G)\rangle$ generated over $K$ by $K Q(f ; G)$ is called a $G$-primitive extension. It follows from [Ko] that $L$ is a strongly normal extension of $K$. Conversely by [Ko] a strongly normal extension of $K$ is obtained by a combination of $G$-primitive extensions. Therefore $K Q=\{f \mid$ meromorphic function on a subdomain of $D$ depending on $f$ such that $f$ is contained in a finite tower of strongly normal extensions; there exist fields $K_{0}=K \subset K_{1} \subset K_{2} \subset \ldots$ $\subset K_{n}$ of meromorphic functions on a subdomain of $D$ such that $K_{i}$ is strongly normal over $K_{i-1}$ for $1 \leqq i \leqq n$ and $\left.x \in K_{n}\right\}$.

ExAmple (2.18). Let us write down explicitly the operation $Q$ for the algebraic groups in (2.5).

(2.18.1) Take $G=\mathbb{G}_{a} \oplus \mathbb{G}_{a}$. Let $f_{1}, f_{2}$ be given functions on $D$. If we admit the golden rule $(\mathrm{O})$, then $f_{1}^{\prime}$ and $f_{2}^{\prime}$ are known functions. Consider now $f: D \rightarrow \mathbb{C} \oplus \mathbb{C}=$ Lie algebra of $G$ defined by $\left.z \mapsto\left(f_{1}^{\prime} z\right), f_{2}^{\prime}(z)\right)$. Then $z \mapsto F(z)=\left(f_{1}(z), f_{2}(z)\right)$ is an integral of $f$. Let $(x, y)$ be the coordinate on $\mathbb{G}_{a} \times \mathbb{G}_{a}$. Then $(x+y) \circ F=f_{1}+f_{2},(x y) \circ F=f_{1} f_{2}$ and if $f_{2} \neq 0,(x / y) \circ F$ $=f_{1} / f_{2}$. Therefore $f_{1}+f_{2}, f_{1} f_{2}, f_{1} / f_{2}$ are obtain from $S=\left\{f_{1}, f_{2}\right\}$ by the $Q$ operation and $(\mathrm{O})$. Namely if we admit $(\mathrm{O})$, the permissible operation (P1) is a special case of the operation $Q$.

(2.18.2) Take $G=\mathbb{G}_{a}$ and let $f$ be a given function on a domain $D$. An integral $F$ of $f: D \rightarrow \mathbb{C}$ is a quadrature $\int f d z$ as we saw in example (2.5.1). Thus in the definition of $Q$, if we take the coordinate $x$ of $\mathbb{G}_{a}$. $x \circ F=F(z)$ is a newly obtained function by the operation $Q$. In other words, the quadrature is a special case of the operation $Q$.

(2.18.3) Now let us assume $G=\mathbb{G}_{m}$ and a holomorphic function $f: D \rightarrow \mathbb{C}$ is given. As we saw in example (2.5.2), an integral is a solution of the differential equation $F^{\prime}(z) / f(z)=f(z)$. Hence if we take the coordinate $x$ of $\mathbb{G}_{m}=\operatorname{Spec}\left(\mathbb{C}\left[x, x^{-1}\right]\right)$ as $\varphi$ in the definition of the operation $Q$, we get $x \circ F=F(z)$. Therefore for known $f$, we can solve the homogeneous linear differential equation $y^{\prime}=f y$. 
(2.18.4) More generally if take $G=G L_{n}(\mathbb{C})$, then it follows from example (2.5.3) that for known functions $a_{i j}(z): D \rightarrow \mathbb{C}$, we can solve the linear differential equation $\left(y_{i j}^{\prime}\right)=\left(y_{i j}\right)\left(a_{i j}(z)\right)$.

(2.18.5) Let us now assume that $G$ is an abelian variety as in example (2.5.4). Let $f_{i}: D \rightarrow \mathbb{C}(1 \leqq i \leqq n)$ be holomorphic functions. An integral of $\left(f_{1}, f_{2}, \cdots, f_{n}\right): D \rightarrow g \cong \mathbb{C}^{n}$ is given by

$$
\left(\int f_{1} d z, \int f_{2} d z, \cdots, \int f_{n} d z\right): D \rightarrow \mathbb{C}^{n}
$$

Therefore using the notation of example (1.2.5), if we take a rational function $\varphi$ on $G, \varphi \circ p \circ \bar{F}$ is a function obtained by the operation $Q$. In particular, let $g_{i}: D \rightarrow \mathbb{C}$ be given holomorphic functions. If we put $g_{i}^{\prime}=f_{i}(1 \leqq i \leqq n)$, then we can take $\bar{F}=\left(g_{1}, g_{2}, \cdots, g_{n}\right)$. Hence for a rational function $\varphi$ on $G \varphi \circ p \circ \bar{F}=\varphi \circ p \circ\left(g_{1}, g_{2}, \cdots, g_{n}\right)$ is a new function obtained by $Q$. We have proved that the operation (P4) is a special case of the operation $Q$ if we allow the operation $(0)$.

Theorem (2.19). Let $S$ be a set of meromorphic functions on a domain $D \subset \mathbb{C}$. Then $S P=S Q$.

Proof. By the operations (P1) and (P3) the constant functions are in $S P$. For the same reason, examples (2.18.1) and (2.18.2) show that $S Q$ contains all the constant functions. Therefore we may assume that $S$ contains the constant functions.

Now the inclusion $S P \subset S Q$ follows from example (2.18), what we have just shown above and from the following

Lemma (2.20). Let $a_{1}(x), a_{2}(x), \cdots, a_{n}(x)$ be a holomorphic functions on a domain $D$. Then the algebroid function $f(x)$, or a solution of an algebraic equation $f^{n}+a_{1} f^{n-1}+\cdots+a_{n}=0$ is a solution of a linear differential equation $d^{m} f / d x^{m}+b_{1}\left(d^{m-1} f / d x^{m-1}\right)+\cdots+b_{m}=0(m \geqq 1)$ such that $b_{i} \in K=\mathbb{C}\left(a_{1}(x), a_{2}(x), \cdots, a_{n}(x), a_{1}^{\prime}(x), a_{2}^{\prime}(x), \cdots, a_{n}^{\prime}(x), a_{i}^{\prime \prime}(x), \cdots\right), 0 \leqq i$ $\leqq m$; namely $b_{i}$ is rational function of $a_{j}$ 's and their derivatives. In par. ticular, the permissible operation (P2) is a consequence of the operations (O), (P1), (P4).

Proof of Lemma. Let us fix a point $z_{0} \in D$ such that the equation

$$
X^{n}+a_{1}\left(z_{0}\right) X^{n-1}+\cdots+a_{n}\left(z_{0}\right)=0
$$


has a simple root $\alpha_{0}$. Taking a small circle $c_{\alpha_{0}}$ centered at $\alpha_{0}$, we can express

$$
\alpha_{0}=\frac{1}{2 \pi i} \int_{c_{\alpha_{0}}} t \frac{\left(t^{n}+a_{1}\left(z_{0}\right) t^{n-1}+\cdots+a_{n}\left(z_{0}\right)\right)^{\prime}}{t^{n}+a_{1}\left(z_{0}\right) t^{n+1}+\cdots+a_{n}\left(z_{0}\right)} d t
$$

(cf. for example Chap VIII, §6, [Di]).

Therefore we may assume

$$
f(z)=\frac{1}{2 \pi i} \int_{c_{\alpha_{0}}} t \frac{n t^{n-1}+(n-1) a_{1}(z) t^{n-2}+\cdots+a_{n-1}(z)}{t^{n}+a_{1}(z) t^{n-1}+\cdots+a_{n}(z)} d t .
$$

Let us look for a homogeneous linear differential equation satisfied by $f(z)$ with coefficients in $K$. We put $F=F(z, t)=t^{n}+a_{1}(z) t^{n-1}+\cdots+$ $a_{n}(t)$. We have

$$
\frac{d f}{d z}=\frac{1}{2 \pi i} \int_{c_{\alpha_{0}}} t \frac{F_{t, z} F-F_{t} F_{z}}{F(z, t)^{2}} d t
$$

where

$$
F_{t}=\frac{\partial F}{\partial t}, \quad F_{z}=\frac{\partial F}{\partial z} \quad \text { and } \quad F_{t, z} \frac{\partial^{2} F}{\partial t \partial z}
$$

We work in the polynomial ring $K[t]$. As we may assume the equation $t^{n}+a_{1} t^{n-1}+\cdots+a_{n}=0$ is irreducible in $\mathbb{C}\left(a_{1}, a_{2}, \cdots, a_{n}\right)[t]$, we have $\left(F_{t}, F\right)=1$. Namely there exists polynomials $A(t), B(t)$ in $K[t]$ such that $A F+B F_{t}=1$. Let $V=\left\{\left(G / F^{m}\right) d t \mid G \in K[x]\right\}$, which is a $K$-vector space of rational 1-forms on the affine line $\mathbb{A}_{K}^{1}$. We need

Sublemma (2.20.1). Let $G(t) \in K[t]$ and $m$ be an integer $\geqq 1$. Then there exist a polynomial $H$ such that $\operatorname{deg}_{t} H \leqq n-1,\left(G / F^{m}\right) d t-(H / F) d t$ is exact: Namely there exist an $I \in K(t)$ such that $\left(G / F^{m}\right) d t-(H / F) d t=$ $d I(=(\partial I / \partial t) d t)$.

Proof of Sublemma. Let $\varphi \in K[t]$. Then we have $\left(\varphi^{\prime} / F^{m}\right)=\varphi^{\prime} / F^{m}+$ $\varphi(-m)\left(F^{\prime} / F^{m+1}\right)$, where the symbol ' means $\partial / \partial t$. Therefore

$$
\frac{\varphi^{\prime}}{F^{m}} d t \equiv-\frac{m \varphi F^{\prime}}{F^{m+1}} d t \bmod (d(K(t)))
$$

Hence if $m \geqq 2$, we get

$$
\frac{G}{F^{m}} d t=\frac{\left(A F+B F^{\prime}\right) G}{F^{m}} \cdot d t=\frac{A}{F^{m-1}} d t+\frac{B F^{\prime} G}{F^{m}} d t
$$




$$
\equiv \frac{A}{F^{m-1}} d t-\frac{1}{m-1} \frac{(B G)^{\prime}}{F^{m-1}} d t \bmod (d(K(t))) \quad \text { by }(2.20 .2)
$$

By repeating this, we can find a polynomial $I \in K[t]$ such that $\left(\left(G / F^{m}\right)-\right.$ $I / F) d t$ is exact. Now if we devide $I$ by $F$, we get

$$
\frac{G}{F^{m}} d t \equiv\left(J+\frac{H}{F}\right) d t \bmod (d(K(t)))
$$

for a suitable polynomials $J, H \in K[t]$ with $\operatorname{deg}_{t} H \leqq n-1$. Since $J d t$ is exact, Sublemma is proved.

Now let us come back to the proof of the lemma. Let us put $\tilde{f}(z)=$ $t\left(F_{t} / F\right) d t$. Then we have

$$
\frac{d^{i} \tilde{f}}{d z^{i}}=\frac{G_{i}}{F^{i+1}} d t \quad \text { with } G_{i} \in K[t] .
$$

Therefore by Sumlemma (2.20.1), there exist $H_{i} \in K[t](0 \leqq i \leqq n)$ such that $\operatorname{deg}_{t} H_{i} \leqq n-1$ and such that

$$
\frac{d^{i} \tilde{f}}{d z^{i}}=\frac{G_{i}}{F^{i+1}} d t \equiv \frac{H_{i}}{F} d t \bmod (d(K(t)))
$$

As the dimension of the $K$-vector space $\left\{H|F| H \in K[t], \operatorname{deg}_{t} H \leqq n-1\right\}$ is $n$, there exists a non-trivial linear relation between $n+1$ elements $H_{i} / F$ and we have non-trivial linear relation

$$
\beta_{0}(z) \frac{d^{n} \tilde{f}}{d z^{n}}+\beta_{1}(z) \frac{d^{n-1} \tilde{f}}{d z^{n-1}}+\cdots+\beta_{n}(z) \tilde{f} \equiv 0 \bmod (d(K(t))),
$$

with $\beta_{j}(z) \in K$. The integration $\frac{1}{2 \pi i} \int_{c_{a_{0}}}$ applied on (2.20.3) now gives the lemma since for any $g \in d(K(t)) \frac{1}{2 \pi i} \int_{c_{\alpha_{0}}} g d t=0$.

Now let us prove $S P \supset S Q$. Let us study the operation $Q$ for a linear algebraic group $G$ and show that in this case the operation $Q$ is the resolution of a linear differential equation as in (P5) and hence is a special case of permissible operations. In fact, we fix a closed immersion $\rho: G \rightarrow G L_{m}(\mathbb{C})$. Let $f_{1}, f_{2}, \cdots, f_{n} \in S$ be given. Let $\left(f_{1}, f_{2}, \cdots, f_{n}\right): D \rightarrow \mathbb{C}^{n}$ $\cong \mathfrak{g}$ be a holomorphic map to the Lie algebra $\mathfrak{g}$ and $F: D \rightarrow G$ be an integral of $\left(f_{1}, f_{2}, \cdots, f_{n}\right)$. Let us denote by $H$ the composite $\rho \circ F: D \rightarrow$ $G L_{m}(\mathbb{C})$. We decompose the Lie algebra $\mathfrak{g l}_{m}$ of $G L_{m}(\mathbb{C})$ as the direct sum of vector spaces $\mathfrak{g l}_{m}=\mathfrak{g} \oplus V$. Then the derivative $H^{\prime}$ of $H$ is given by 


$$
\left(f_{1}, f_{2}, \cdots, f_{n}, 0, \cdots, 0\right): D \rightarrow V=\mathfrak{g l}_{n} .
$$

Thus by the definition of the operation $Q$ for $\left(f_{1}, f_{2}, \cdots, f_{n}, 0, \cdots, 0\right)$ and $G=G L_{m}(\mathbb{C})$, for any rational function $\varphi$ on $G L_{m}(\mathbb{C}), \varphi \circ H: D \rightarrow$ $G L_{m}(\mathbb{C}) \cdots \rightarrow \mathbb{C}$ is a new known functions. Since $\varphi \circ H=\varphi \circ \rho \circ F$ and any rational function on $G$ is the restriction of a rational function on $G L_{m}(\mathbb{C})$, we have proved the desired result in example (2.18.4). We have already seen in (2.18.5) that the operation $Q$ for an abelian variety is equivalent to (P5) combined with (O).

Let us now study the general case. It is known that their exists a closed, connected normal linear subgroup $H$ of $G$ such that the quotient $G / H$ is an abelian variety $A$ :

$$
1 \longrightarrow H \stackrel{i}{\longrightarrow} G \stackrel{p}{\longrightarrow} A \longrightarrow 1
$$

(see for example [B]).

We show using this structure theorem that the operation $Q$ for $G$ reduces to the linear case and the abelian case. Let $f_{1}, f_{2}, \cdots, f_{n} \in S$ be given holomorphic functions on $D$ such that we get $f=\left(f_{1}, f_{2}, \cdots, f_{n}\right): D$ $\rightarrow \mathbb{C}^{n} \cong \mathfrak{g}$. Let $F$ be an integral of $f=\left(f_{1}, f_{2}, \cdots, f_{n}\right): D \rightarrow \mathfrak{g}$. Then $T=p \circ F$ is an integral of $p_{*} \circ f: D \rightarrow \mathfrak{g} \rightarrow \mathfrak{a}$ by Proposition (2.9), where we denote by $a$ the Lie algebra of $A$. Since $p_{*} \circ f: D \rightarrow \mathfrak{a}$ is written by using functions in $\mathbb{C}\left(f_{1}, f_{2}, \cdots, f_{n}\right)$, for any rational function $\varphi$ on $A \varphi \circ T$ $=\varphi \circ(p \circ F)$ are known function by the permissible operation (P5). To make the proof comprehensive, let us first assume that there exists a rational section $s$ of $p: s$ is a rational map of $A$ to $G$ such that $p \circ s=\operatorname{Id}_{A}$. We may assume that $s$ is regular on $T(D)$. Let us put $U=s \circ T: D \rightarrow$ $A \stackrel{s}{\rightarrow} G$. Then $U$ has the following properties:

(2.22.1) for any rational function $\psi$ on $G, \psi \circ U=\psi \circ(s \circ T)=(\psi \circ s) \circ T$ is a known function by the operations (O), (P1), (P5) from $S$;

(2.22.2) $U^{-1} F(z)=U(z)^{-1} \cdot F(z)$ (the -1 and the multiplication are taken with respect to the values in $G$ ) is contained in $H$.

Sublemma (2.23). The derivative of $U^{-1} F: D \rightarrow H$ is given by known functions obtained from $S$ by permissible operations (O), (P1), , , (P5).

Proof of Sublemma. We may assume that $U: D \rightarrow G$ factors through an affine open set $X \subset G$. Let $X \longrightarrow \mathbb{A}^{l}$ be an embedding given by $x \mapsto$ $\left(z_{1}(x), z_{2}(x), \cdots, z_{l}(x)\right)$. Then by (2.22.1) each coordinate of $U: D \rightarrow X \smile$ 
$\mathbb{A}^{l}$ is a known function by $(\mathrm{O}),(\mathrm{P} 1), \cdots$ and (P5). Thus $U^{-1}=V$ is also expressed by known functions since $G$ is an algebraic group over $\mathbb{C}$ and hence also the derivative $V^{\prime}$. Therefore we may assume replacing $D$ that there exists a subfield $K \subset S P$ consisting of meromorphic functions on $D$ such that $\tau(V), \tau(F) \in K \otimes_{\mathrm{C}} \mathfrak{g}$ and $V$ defines a $K$-rational point $P(V)$ of $G \otimes_{\mathrm{c}} K(D)$.

We show that the derivation $\tau(V F): O_{G \otimes \mathbb{C} K(D), 1} \rightarrow K(D)$ maps $f \in O_{G, 1}$ to $K$. In fact, by definition

$$
\begin{aligned}
\tau(V F)(f)= & \lim _{h \rightarrow 0} \frac{f\left(V F(z+h)\left(V F(z)^{-1}\right)-f(1)\right.}{h} \\
= & \lim _{h \rightarrow 0} \frac{f\left(V(z+h) F(z+h) F(z)^{-1} V(z)^{-1}\right)-f\left(V(z+h) V(z)^{-1}\right)}{h} \\
& +\frac{f(V(z+h) V(z))-f(1)}{h} .
\end{aligned}
$$

The latter term is equal to $\tau(V)(f)$ hence in $K$ by Lemma (2.13). It is sufficient to show that

$$
\lim _{h \rightarrow 0} \frac{f\left(V(z+h) F(z+h) F(z)^{-1} V(z)^{-1}\right)-f\left(V(z+h) V(z)^{-1}\right)}{h}
$$

is in $K$. First we show that

$$
\begin{gathered}
\lim _{h \rightarrow 0} \frac{f\left(V(z+h) F(z+h) F(z)^{-1} V(z)^{-1}\right)-f\left(V(z+h) V(z)^{-1}\right)}{h} \\
=\lim _{h \rightarrow 0} \frac{f\left(V(z) F(z+h) F(z)^{-1} V(z)^{-1}\right)-f(1)}{h} .
\end{gathered}
$$

To this end it is enough to show

$$
\begin{gathered}
f\left(V(z+h) F(z+h) F(z)^{-1} V(z)^{-1}\right)-f\left(V(z+h) V(z)^{-1}\right) \\
-f\left(V(z) F(z+h) F(z)^{-1} V(z)^{-1}\right)+f(1)=h^{2} \\
\quad \times \text { (holomorphic function in }(z, h) \in D \times E
\end{gathered}
$$

where $E$ is a neighbourhood of $0 \in \mathbb{C}$. Let us put

$$
\varphi(g, t, h)=f\left(g V(t) F(t+h) F^{-1}(t) V^{-1}(t)\right)-f(g)
$$

for $g$ in a small neighbourhood $W$ of $1 \in G . \quad \varphi(g, t, h)$ is a holomorphic function in $g, t, h$. Since $\varphi(g, t, 0)=0, \varphi(g, t, h)=h \hat{\varphi}(g, t, h)$ with $\hat{\varphi}$ holomorphic. The left side of (2.25) is equal to

$$
\varphi\left(V(t+h) V(t)^{-1}, t, h\right)-\varphi(1, t, h)=h\left(\tilde{\varphi}\left(V(t+h) V(t)^{-1}, t, h\right)-\tilde{\varphi}(1, t . h)\right) .
$$


Since

$$
\begin{gathered}
\tilde{\varphi}\left(V(t+h) V(t)^{-1}, t, h\right)-\hat{\varphi}(1, t, h) \longrightarrow 0 \text { as } h \longrightarrow 0, \\
\frac{\tilde{\varphi}\left(V(t+h) V(t)^{-1}, t, h\right)-\tilde{\varphi}(1, t, h)}{h}
\end{gathered}
$$

is holomorphic and hence

$$
\frac{\varphi\left(V(t+h) V(t)^{-1}, t, h\right)-\varphi(1, t, h)}{h^{2}}
$$

is holomorphic as desired. It remains to show that

$$
\lim _{h \rightarrow 0} \frac{f\left(V(z) F(z+h) F(z)^{-1} V(z)^{-1}\right)-f(1)}{h} \in K .
$$

$V: D \rightarrow G$ is a $K$-valued point and $\tau(F)$ is in $g \otimes_{c} K$. Therefore Ad $V(z)(\tau(F))$ is in $\mathfrak{g} \otimes_{c} K$ by Lemma (2.12).

$$
\begin{aligned}
& \lim _{h \rightarrow 0} \frac{f\left(V(z) F(z+h) F(z)^{-1} V(z)^{-1}\right)-f(1)}{h} \\
= & \lim _{h \rightarrow 0} \frac{f\left(V(z) F(z+h) V(z)^{-1} V(z) F(z)^{-1} V(z)^{-1}\right)-f(1)}{h} \\
= & \operatorname{Ad} V(z)(\tau(F))(f) \in K .
\end{aligned}
$$

It follows from the sublemma and from what we have shown for linear algebraic groups that $\varphi\left(U^{-1} \cdot F(z)\right)$ is a known function by (0), (P1), $\cdots$ and (P5) for any rational function $\varphi$ on $H$ hence on $G$. In particular, we have shown that if take an affine open set $X \subset G$ such that $X \longrightarrow \mathbb{A}^{l}$ is given by $x \mapsto\left(z_{1}(x), z_{2}(x), \cdots, z_{l}(x)\right.$ ), each coordinates of $U^{-1} F$ (resp. $\left.U^{-1}\right): D \rightarrow X \subset \mathbb{A}^{l}$ is given by a known function by the permissible operations. Since $G$ is an algebraic group, each coordinate of the product $F=U\left(U^{-1} F\right)$ is also written by a known function by (P1). Since a rational function $\psi$ on $G$ is a rational function of $z_{i}^{\prime}$ 's, $\psi \circ F$ is a rational function of the $\left(\varphi \circ z_{i}\right)$ 's hence known function by (P1).

Let us now treat the most general case where we can not find a rational section $s$ of $p$. We know that there exists an irreducible (reduced) subvariety $B$ of $G$ such that the restriction $p \mid B: B \rightarrow A$ is finite. Since the extension $k(B) / k(A)$ is algebraic and since we are allowed to use operation (P2), for any rational function $\varphi$ on $B$, multi-valued function $\varphi \circ(p \mid B)^{-1} \circ(p \circ F)$ is a known function by permissible operations. Since for any rational function $\varphi$ on $G$ its restriction on $B$ is a rational func- 
tion on $B$, therefore $\varphi \circ(p \mid B)^{-1} \circ(p \circ F)$ is a known function. We have thus proved that there exists a holomorphic map $U: D \rightarrow G$ with the following properties:

(2.26.1) For any rational function $\psi$ on $G, \psi \circ U$ is a known function by the operations (O), (P1), .., (P5).

(2.26.1) The image of $U^{-1} F: D \rightarrow G$ is contained in $H$.

Now the argument of the preceding case gives the Theorem.

In view of Theorem (2.19) it is natural to adopt

Definition (2.27). Let $\mathbb{C}$ be the set of constant functions on the plane $\mathbb{C}$. We call an element of $\mathbb{C P}=\mathbb{C Q}$ a classical function.

The polynomials of one variable, $e^{x}, \log x$, the Weierstassian functions $\rho, \zeta, \sigma$, the hypergeometric function, and the Bessel functions are, for example classical functions.

\section{§ 3. Solvability theorem of Painlevé (finite type case)}

We need algebraic tools to make the argument rigorous and comprehensive. So let us start with preliminary results in the algebraic geometry. Let $R$ be a $\mathbb{C}$-algebra of holomorphic functions on a domain $D$ in $\mathbb{C}$. We are interested in the local properties of holomorphic functions. Hence in the following argument whenever necessary we replace $D$ by a subdomain of $D$. Let $f_{1}\left(t ; x_{1}, x_{2}, \cdots, x_{n}\right), f_{2}\left(t ; x_{1}, x_{2}, \cdots, x_{n}\right), \cdots$, $f_{m}\left(t ; x_{1}, x_{2}, \cdots, x_{n}\right) \in R\left[x_{1}, x_{2}, \cdots, x_{n}\right]$ be polynomials with coefficients in $R$, where we denote by $x$ the coordinate on $D$. We denote by $X$ the analytic subspace in $D \times \mathbb{A}_{\mathrm{c}}^{n}$ defined by $f_{1}, f_{2}, \cdots, f_{n}$ : namely $X=\left\{\left(x ; z_{1}, z_{2}\right.\right.$, $\left.\cdots, z_{n}\right) \in D \times \mathbb{A}_{c}^{n} \mid f_{i}\left(x ; z_{1}, z_{2}, \cdots, z_{n}\right)=0$ for $\left.1 \leq i \leq m\right\}$. We have a morphism $p: X \rightarrow D$ induced by the projection. Replacing $D$ by a small subdomain if necessary and adding finitely many polynomials to $\left\{f_{1}, f_{2}, \cdots, f_{n}\right\}$, we may assume that $X$ is reduced. We denote the fibre $p^{-1}(x)$ by $X_{x}$ for $x \in D$. We say that $X$ is defined over $R$.

Let $K$ be the quotient field of $R$. If an element $f$ of a over field of $K$ and if $f$ is algebraic over $K$, then there exist $a_{0}, a_{1}, a_{2}, \cdots, a_{n} \in R$ such that $a_{0} \neq 0, a_{0} f^{n}+a_{1} f^{n-1}+\cdots+a_{n} f=0$. Therefore $f$ can be considered as an algebroid function. In particular by iterating operation (P2) and by arithmetic operations (P1), we get an algebraic closure $\bar{K}$ from $K$ (see $\S_{\triangleleft} 2$ ).

We need 
Lemma (3.1). We keep the notation above. If we replace $D$ by a suitable, subdomain $D^{\prime}$, there exist a finitely many elements $b_{1}, b_{2}, \cdots, b_{l} \in \bar{K}$ and analytic subvarieties $Y_{1}, Y_{2}, \cdots, Y_{s}$ of $D^{\prime} \times \mathbb{A}_{\mathrm{c}}^{n}$ defined over $R^{\prime}=$ $R\left[b_{1}, b_{2}, \cdots, b_{l}\right]$ such that $X_{x}=\bigcup_{i=1}^{s} Y_{i x} \subset D^{\prime} \times \mathbb{A}_{c}^{n}$ and the $Y_{i}$ 's are the irreducible components of $X_{x}$ for any $x \in D^{\prime}$.

Proof. Since only finitely many $f_{i}$ 's are involved, we may assume that the ring $R$ is of finte type: $R=\mathbb{C}\left[c_{1}, c_{2}, \cdots, c_{d}\right]$ where $c_{i}$ is a holomorphic function on $D$. Then $\operatorname{Spec} R \subset \mathbb{A}_{\mathrm{C}}^{d}$ and the holomorphic map $\varphi: D \rightarrow \mathbb{A}^{d}$ defined by $\varphi(x)=\left(c_{1}(x), c_{2}(x), \cdots, c_{d}(x)\right) \in \mathbb{A}_{\mathrm{c}}^{d}$ factors through $\operatorname{Spec} R \subset \mathbb{A}_{\mathrm{c}}^{d}$ and hence defines $\varphi: D \rightarrow \operatorname{Spec} R$. It follows from Lemma (1.25) of Part I that the image $\varphi(D)$ is not contained in any closed algebraic subvariety of Spec $R$ except for Spec $R$ itself. Let us now put $\mathscr{X}=\left\{y \in \operatorname{Spec} R \times \mathbb{A}_{\mathrm{C}}^{n}=\right.$ $\mathbb{A}_{R}^{n} \mid f_{i}\left(t ; y_{1}, y_{2}, \cdots, y_{n}\right)=0$ for $\left.1 \leq i \leq m\right\}$. Then $X=\mathscr{X} \times_{\operatorname{Spec} R} D$. Let us now consider $\mathscr{X} \times_{R} \bar{K}=\mathscr{X}_{\bar{K}}$ and decompose the variety $\mathscr{X}_{\bar{K}}$ over $\bar{K}$ to the irreducible components $\mathscr{X}_{\bar{K}}=\mathscr{Y}_{1} \cup \mathscr{Y}_{2} \cup \cdots \cup \mathscr{Y}_{s} \subset \mathbb{A}_{\bar{K}}^{n}$. Then $y_{1}, y_{2}, \cdots, y_{s}$ are defined over a certain finite extension $R^{\prime}=R\left[b_{1}, b_{2}, \cdots, b_{l}\right]$ and they are absolutely irreducible. Therefore there exists a Zariski open subset $\phi \neq U$ of $\operatorname{Spec} R^{\prime}$ such that $\mathscr{Y}_{i x}$ is irreducible and $\mathscr{X}_{x}=\bigcup_{i=1}^{l} \mathscr{Y}_{i x}$ for any $x \in U$ for $1 \leq i \leq S$. Now we have $R^{\prime}=R\left[b_{1}, b_{2}, \cdots, b_{l}\right]=\mathbb{C}\left[c_{1}, c_{2}, \cdots\right.$, $\left.c_{d}, b_{1}, b_{2}, \cdots, b_{l}\right]$ and $b_{1}, b_{2}, \cdots, b_{l}, c_{1}, c_{2}, \cdots, c_{d}$ are holomorphic functions over a certain subdomains. As we remarked above, if define $\varphi^{\prime}: D^{\prime} \rightarrow$ Spec $R^{\prime}$ as for $D$ and $R^{\prime}$, the image $\varphi^{\prime}\left(D^{\prime}\right)$ is not contained in any proper closed algebraic subvariety of Spec $R^{\prime}$. In particular $\varphi\left(D^{\prime}\right) \cap U \neq \varnothing$. Hence if we put $Y_{i}=\mathscr{Y}_{i} \times{ }_{D^{\prime}}$ Spec $R^{\prime}, Y$ satisfies the requirements.

Let $R$ be a $\mathbb{C}$-algebra of holomorphic functions on a domain $D$ in $\mathbb{C}$. Let $F\left(t ; X_{0}, X_{1}, X_{2}, \cdots, X_{n}\right) \in R\left[X_{0}, X_{1}, \cdots, X_{n}\right]$ be a polynomial with coefficients in $R$, with $\partial F / \partial X_{n} \not \equiv 0$. We consider an algebraic ordinary differential equation

$$
F\left(t ; y(t), y^{\prime}(t), \cdots, y^{(n)}(t)\right)=0 \text {. }
$$

It follows from the argument above that using permissible operations (0), (P1), $\cdots,(\mathrm{P} 5)$, we may assume that the subvariety $X_{t_{0}}=\left\{\left(z_{0}, z_{1}, \cdots, z_{n}\right) \in\right.$ $\left.\mathbb{A}_{\mathrm{c}}^{n} \mid F\left(t_{0} ; z_{0}, z_{1}, \cdots, z_{n}\right)=0\right\}$ of $\mathbb{A}^{n}$ is irreducible for any $t_{0} \in D$ and $F$ is absolutely irreducible. We denote by $X$ the analytic variety $\left\{\left(t ; z_{0}, z_{1}, \cdots, z_{n}\right)\right.$ $\left.\in D \times \mathbb{A}^{n} \mid F\left(t ; z_{0}, z_{1}, \cdots, z_{n}\right)=0\right\} . \quad$ Let $\mathscr{X}=\left\{\left(t ; y_{0}, y_{1}, \cdots, y_{n}\right) \in \operatorname{Spec} R \times\right.$ $\left.\mathbb{A}_{\mathrm{c}}^{n} \mid F\left(t ; y_{0}, y_{1}, \cdots, y_{n}\right)=0\right\}$. We have a morphism $q: \mathscr{X} \rightarrow \operatorname{Spec} R$ and as we have seen above $X=\mathscr{X} \times{ }_{D} \operatorname{Spec} R$. We call $\mathscr{X}$ the family of alge- 
braic varieties associated to $X$. We denote by $X^{0}$ the open set $\left\{\left(t ; z_{0}, z_{1}\right.\right.$, $\left.\left.\cdots, z_{n}\right) \in X \mid\left(\partial F / \partial X_{n}\right)\left(t ; z_{0}, z_{1}, \cdots, z_{n}\right) \neq 0\right\}$. Then $X^{0}$ is a complex manifold. The general solution of the differential equation (3.2) is equivalent to finding the solution $\left(t ; f_{0}(t), f_{1}(t), \cdots, f_{n}(t)\right)=\left(t ; x_{0}, x_{1}, \cdots, x_{n}\right)$ of the following regular system of Pfaffian equations of corank 1 on $X^{0}$;

$$
\begin{aligned}
& d x_{i}=x_{i+1} d t \quad(0 \leq i \leq n-1) \\
& d x_{n}=A\left(t ; x_{0}, x_{1}, \cdots, x_{n}\right) d t
\end{aligned}
$$

where

$$
A\left(t ; x_{0}, x_{1}, \cdots, x_{n}\right)=-\left(\frac{\partial F}{\partial t}+\sum_{i=0}^{n} x_{i-1} \frac{\partial F}{\partial X}\right) /\left(\frac{\partial F}{\partial X_{n}}\right)
$$

(cf. $\S 1)$. The dual to (3.3) is the vector field

$$
\Delta=\frac{\partial}{t \partial}+X_{1} \frac{\partial}{\partial X_{0}}+X_{2} \frac{\partial}{\partial X_{1}}+\cdots+X_{n} \frac{\hat{o}}{\partial X_{n-1}}+A \frac{\partial}{\partial X_{n}} .
$$

Namely let $y(t)$ be a solution of the equation (3.2). The integral curve $\left(t ; y(t), y^{\prime}(t), \cdots, y^{(n)}(t)\right)$ of the Pfaffian system (3.3) defines the vector field (3.4) and $\Delta(F)=0$.

Replacing $D$ by a subdomain, we may assume that for any $t_{0} \in D$, there exists a ponit $\left(z_{0}, z_{1}, \cdots, z_{n}\right) \in \mathbb{A}^{n+1}$ with $F\left(t_{0} ; z_{0}, z_{1}, \cdots, z_{n}\right)=0$ such that $\left(\partial F / \partial X_{n}\right)\left(t_{0} ; z_{0}, z_{1}, \cdots, z_{n}\right) \neq 0$. Let $W=\left\{\left(z_{0}, z_{1}, \cdots, z_{n}\right) \in \mathbb{A}^{n+1} \mid F\left(t_{0} ; z_{0}, z_{1}\right.\right.$, $\left.\left.\cdots, z_{n}\right)=0,\left(\partial F / \partial X_{n}\right)\left(t_{0} ; z_{0}, z_{1}, \cdots, z_{n}\right) \neq 0\right\}$. If $\left(z_{0}, z_{1}, \cdots, z_{n}\right) \in W \subset X_{t_{0}}$, then $X$ is non-singular at $\left(t_{0} ; z_{0}, z_{1}, \cdots, z_{n}\right)$ and there exists the unique holomorphic solution $y\left(t_{0} ; z_{0}, z_{1}, \cdots, z_{n} ; t\right)$ of the differential equation (3.2) with $y^{(i)}\left(t_{0} ; z_{0}, z_{1}, \cdots, z_{n} ; t_{0}\right)=z_{i}$ for $0 \leq i \leq n$. The $y^{(i)}\left(t_{0} ; z_{0}, z_{1}, \cdots, z_{n} ; t\right)$ are holomorphic with respect to $\left(z_{0}, z_{1}, \cdots, z_{n}\right) \in W$.

We denote by $\bar{X}$ (resp. $\overline{\mathscr{X}}, \bar{X}_{t}$ ) the projectification or the closure of $X \subset D \times \mathbb{A}^{n}$ (resp. $\left.\mathscr{X} \subset D \times \mathbb{A}^{n}, X_{a} \subset \mathbb{A}^{n}\right)$ in $D \times \mathbb{P}^{n}$ (resp. $D \times \mathbb{P}^{n}, \mathbb{P}^{n}$ ).

When $D^{\prime}$ is a connected open subset of $D$, we denote by $X^{\prime}$ the fibre product $X \times{ }_{D} D^{\prime}$. If $R^{\prime}$ is an over ring of $R$, we denote by $\mathscr{X}^{\prime}$ the scheme $\mathscr{X} \otimes_{R} R^{\prime}$ over $R^{\prime}$.

Lemma (3.5). The following conditions are equivalent.

(i) There exist a point $t_{0} \in D$, an open neighbourhood $D^{\prime}$ of $t_{0}$, a non-empty open set $V$ of $X_{t_{0}} \times D$ and polynomials $C_{i}\left(t ; x_{0}, x_{1}, \cdots, x_{n}\right)$, $D_{i}\left(t ; x_{0}, x_{1}, \cdots, x_{n}\right)$ in $x_{0}, x_{1}, \cdots, x_{n}$ with coefficients in a ring of holomorphic functions on $D^{\prime}$ such that

(a) if $\left(t ; z_{0}, z_{1}, \cdots, z_{n}\right) \in V$, then $\left(\partial F / \partial X_{n}\right)\left(t_{0} ; z_{0}, z_{1}, \cdots, z_{n}\right) \neq 0$ and 
(b) the solution $y^{(i)}\left(t_{0} ; z_{0}, z_{1}, \cdots, z_{n} ; t\right)$ is regular at $\left(t ; z_{0}, z_{1}, \cdots, z_{n}\right) \in V$ $(0 \leq i \leq n)$ and such that

(c) $y^{(i)}\left(t_{0} ; z_{0}, z_{1}, \cdots, z_{n} ; t\right)=\left(C_{i}\left(t ; z_{0}, z_{1}, \cdots, z_{n}\right)\right) /\left(D_{i}\left(t ; z_{0}, z_{1}, \cdots, z_{n}\right)\right)$ for any $\left(t ; z_{0}, z_{1}, \cdots, z_{n}\right) \in V$ and for all $0 \leq i \leq n$.

(ii) There exists a point $t_{0} \in D$, an open neighbourhood $D^{\prime}$ of $t_{0}$, a nonempty open set $V$ of $X_{t_{0}} \times D$ and polynomial $C\left(t ; x_{0}, x_{1}, \cdots, x_{n}\right), D\left(t ; x_{0}, x_{1}\right.$, $\left.\cdots, x_{n}\right)$ in $x_{0}, x_{1}, \cdots, x_{n}$ with coefficients in a ring of holomorphic functions on $D^{\prime}$ such that (a) if $\left(t ; z_{0}, z_{1}, \cdots, z_{n}\right) \in V$, then $\left(\partial F / \partial X_{n}\right)\left(t_{0} ; z_{0}, z_{1}, \cdots, z_{n}\right) \neq 0$, (b) the solution $y\left(t_{0} ; z_{0}, z_{1}, \cdots, z_{n} ; t\right)$ is regular at $\left(t ; z_{0}, z_{1}, \cdots, z_{n}\right) \in V$ and such that (c) $y\left(t_{0} ; z_{0}, z_{1}, \cdots, z_{n} ; t\right)=\left(C\left(t ; z_{0}, z_{1}, \cdots, z_{n}\right)\right) /\left(D\left(t ; z_{0}, z_{1}, \cdots, z_{n}\right)\right)$ for any $\left(t ; z_{0}, z_{1}, \cdots, z_{n}\right) \in V$.

(iii) There exist a point $t_{0} \in D$, an open neighbourhood $D^{\prime}$ of $t_{0}$ such that if we denote by $Z$ the set $\left\{\left(t ; x_{0}, x_{1}, \cdots, x_{n}, y\left(t_{0} ; x_{0}, x_{1}, \cdots, x_{n} ; t\right), y^{(1)}\left(t_{0} ; x_{0}, x_{1}\right.\right.\right.$, $\left.\left.\cdots, x_{n} ; t\right), \cdots, y^{(n)}\left(t_{0} ; x_{0}, x_{1}, \cdots, x_{n} ; t\right)\right) \in\left(D^{\prime} \times X_{t_{0}}\right) \times_{D^{\prime}} X^{\prime} \mid\left(\partial F / \partial X_{n}\right)\left(t_{0} ; x_{0}, x_{1}\right.$, $\left.\cdots, x_{n}\right) \neq 0, y^{(i)}\left(t_{0} ; x_{0}, x_{1}, \cdots, x_{n} ; t\right)$ is regular at $\left.\left(x_{0}, x_{1}, \cdots, x_{n} ; t\right) \in X_{t_{0}} \times D^{\prime}\right\}$ the Zariski closude $\bar{Z} \subset\left(D^{\prime} \times \bar{X}_{t_{0}}\right) \times{ }_{D^{\prime}} \bar{X}^{\prime} \subset D^{\prime} \times \bar{X}_{t_{0}} \times \bar{X}^{\prime}$ defines a $D^{\prime}$ bimeromorphic correspondence of $D^{\prime} \times \bar{X}_{t_{0}}$ and $\bar{X}^{\prime}$ (a bimeromorphic map commuting with the projections onto $D$ and inducing a bimeromorphic correspondence between the fibres as in Part I, §1).

Proof. (ii) is a special case of (i). By differentiating with respect to $t$, (ii) implies (i). Therefore (i) and (ii) are equivalent. We may assume that the ring $R$ which contains the coefficients of the differential equation (3.2) is of finite type over $\mathbb{C}$. If the condition (i) is satisfied, then denoting by $R^{\prime}$ the ring obtained by the adjunction of the coefficients of $C_{i}$ 's and $D_{i}$ 's to $R$, we get a rational map $\Phi: X_{t_{0}} \times \operatorname{Spec} R^{\prime} \cdots \rightarrow \mathscr{X} \otimes_{R} R^{\prime}$ $=\mathscr{X}^{\prime}$ over Spec $R^{\prime}$. We show that $\Phi^{-1}$ is also rational. Let $\mathscr{Z} \subset \bar{X}_{t} \times$ Spec $R^{\prime} \times_{\text {Spec } R^{\prime}} \overline{\mathscr{X}} \subset \bar{X}_{t_{0}} \times \operatorname{Spec} R^{\prime} \times \overline{\mathscr{X}}$ be the graph of $\Phi$. We have to show that the projection $p=p_{3}: \mathscr{Z} \rightarrow \bar{X}^{\prime}$ is birational. Let $\left(x_{0}, x_{1}, \cdots, x_{n}\right)$ $\in X_{t_{0}}$ with $\left(\partial F / \partial X_{n}\right)\left(t_{0} ; x_{0}, x_{1}, \cdots, x_{n}\right) \neq 0$. Then around $\left(t_{0} ; x_{0}, x_{1}, \cdots, x_{n}\right) \in X$ for the usual topology, the solution $y\left(t_{0}, x, t\right), y^{(1)}\left(t_{0}, x, t\right), \cdots, y^{(n)}\left(t_{0}, x, t\right)$ or $\Phi$ gives local analytic isomorphism between $X$ and $X_{t_{0}} \times D$. Therefore the map $p: \mathscr{Z} \rightarrow \overline{\mathscr{X}}$ is dominant by Lemma (1.25) of Part I. It is sufficient to show that $p$ is finite and of degree 1. To this end, it is sufficient to show that there exists an open set $U$ of $X_{t_{0}} \times \operatorname{Spec} R^{\prime}$ such that for a general point $z \in \overline{\mathscr{X}}^{\prime}$ we have $\Phi^{-1}(z) \cap U$ consists of one point. Since $\bar{X} \subset \overline{\mathscr{X}}^{\prime}$ is not contained in any proper Zariski closed subset of $\overline{\mathscr{X}}$ by Lemma (1.25) of Part I, it is also sufficient to check this for a dense subset of $\bar{X}$. 
Let $\left(u_{0}, u_{1}, \cdots, u_{n} ; t_{1}\right) \in X \subset \mathbb{A}^{n+1} \times D^{\prime}$ with $\left(\partial F / \partial X_{n}\right)\left(t_{1} ; u_{0}, u_{1}, \cdots, u_{n}\right) \neq 0$ and $D_{i}\left(t_{1} ; u_{0}, u_{1}, \cdots, u_{n}\right) \neq 0$ for $0 \leq i \leq n$. Let us put $U=\left\{(x, s) \in \bar{X}_{t_{0}} \times\right.$ Spec $R^{\prime} \mid D_{i}\left(s ; x_{1}, x_{0}, \cdots, x_{n}\right) \neq 0$ and $\left.\left(\partial F / \partial X_{n}\right)\left(s ; x_{0}, x_{1}, \cdots, x_{n}\right) \neq 0\right\}$. Let $\left(x, s_{1}\right)=\left(x_{0}, x_{1}, \cdots, x_{n} ; s_{1}\right),\left(z, s_{2}\right)=\left(z_{0}, z_{1}, \cdots, z_{n} ; s_{2}\right) \in U$ with $\Phi\left(x, s_{1}\right)=\Phi\left(z, s_{2}\right)$ $=(u, t) \in X$. Then $s_{1}=s_{2}=t_{1}$. We notice that since

$$
y^{(i)}\left(t_{0} ; w_{0}, w_{1}, \cdots, w_{n} ; t\right)=\frac{C_{i}\left(t ; w_{0}, w_{1}, \cdots, w_{n}\right)}{D_{i}\left(t ; w_{0}, w_{1}, \cdots, w_{n}\right)}
$$

on an open set $V$. Therefore if for $\left(v_{0}, v_{1}, v_{2}, \cdots, v_{n}\right) \in X_{t_{0}}$, we have (1) $D_{i}\left(t_{0} ; v_{0}, v_{1}, \cdots, v_{n}\right) \neq 0,0 \leq i \leq n$ and $(2) \quad\left(\partial F / \partial X_{n}\right)\left(t_{0} ; v_{0}, v_{1}, \cdots, v_{n}\right) \neq 0$, then it follows from the unicity of the solution of (3.2) that

$$
y^{(i)}\left(t_{0} ; v_{0}, v_{1}, \cdots, v_{n} ; t\right)=\frac{C_{i}\left(t ; v_{0}, v_{1}, \cdots, v_{n}\right)}{D_{i}\left(t ; v_{0}, v_{1}, \cdots, v_{n}\right)}, \quad 0 \leq i \leq n .
$$

By the uniqueness of the solution of the differential equation $F=0$ at $t=t_{1}$ with initial conditions $u_{0}, u_{1}, \cdots, u_{n}$, we have $y^{(i)}\left(t_{0}, x, t\right)=y^{(i)}\left(t_{0}, z, t\right)$ around $t=t_{1}$ hence for any $t \in D^{\prime}$. Therefore if we put $t=t_{0}$, we get $x=z$. Hence $\mathscr{Z} \subset\left(\bar{X}_{t_{0}} \times \operatorname{Spec} R^{\prime}\right) \times \overline{\mathscr{X}}$ is a birational correspondence. Now (iii) is obtained by taking the fibre product with respect to the map $D^{\prime} \rightarrow \operatorname{Spec} R^{\prime}$.

Assume now that the condition (iii) is satisfied. We denote $D^{\prime}$ by $D$ again. Since $Z \subset D \times \bar{X}_{t_{0}} \times \bar{X} \subset D \times \mathbb{P}^{n+1} \times \mathbb{P}^{n+1}$, we may assume by the relative G.A.G.A. (cf. [G1]) that $Z$ is defined by polynomials $F_{1}(t ; U, V)$, $F_{2}(t ; U, V), \cdots, F_{N}(t ; U, V)$ homogeneous in $U=\left(U_{0}, U_{1}, \cdots, U_{n+1}\right)$ and $V=\left(V_{0}, V_{1}, \cdots, V_{n+1}\right)$ with holomorphic coefficients. Let $R^{\prime}$ be the ring extension of $R$ by the adjunction of coefficients of $F_{i}(1 \leq i \leq N)$. Hence $R^{\prime}$ is of finite type over $R$. We can thus define the scheme $\mathscr{Z} \rightarrow \operatorname{Spec} R^{\prime}$ associated to $Z$. We have a inclusion $i: \mathscr{Z} \rightarrow \mathbb{P}^{n+1} \times \mathbb{P}^{n+1} \times \operatorname{Spec} R^{\prime}$. The projection $p_{13}: \mathbb{P}^{n+1} \times \mathbb{P}^{n+1} \times \operatorname{Spec} R^{\prime} \rightarrow \mathbb{P}^{n+1} \times$ Spec $R^{\prime}$ onto the product of the first and the third factors defines $p_{13} \circ i: \mathscr{Z} \rightarrow \mathbb{P}^{n+1} \times \operatorname{Spec} R^{\prime}$ which factors through $X_{t_{0}} \otimes_{\mathrm{c}} R^{\prime} \subset \mathbb{P}^{m+1} \times \operatorname{Spec} R^{\prime}$. Hence we have a Spec $R^{\prime}$ morphism $f: \mathscr{Z} \rightarrow X_{t_{0}} \otimes_{\mathrm{c}} R^{\prime}$ of algebraic varieties over $\mathbb{C}$. The restriction of $f$ to $D \rightarrow \operatorname{Spec} R^{\prime}$ is the projection $Z \rightarrow D$.

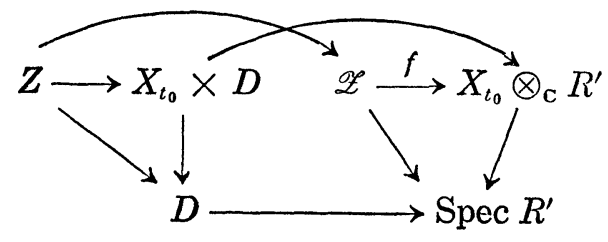


The hypothesis shows that the fibre $f^{-1}(x)$ consists of one point if $x$ is in a non-empty open set for the usual topology of $X_{t_{0}} \otimes D$ which is not contained in no Zariski closed set of the algebraic variety $X_{t_{0}} \otimes_{\mathrm{C}} R^{\prime}$ by Lemma (1.25). Therefore it follows (for example from [M]) that the degree of $f$ is equal to $1 ; f$ is birational. Let $K^{\prime}$ be the quotient field of $R^{\prime}$. The rational function field $\mathbb{C}(\mathscr{Z})$ of the algebraic variety $\mathscr{Z}$ is $K^{\prime}\left(u_{1} / u_{0}, u_{2} / u_{0}, \cdots, u_{n+1} / u_{0}, v_{1} / v_{0}, v_{2} / v_{0}, \cdots, v_{n+1} / v_{0}\right)$, where $u_{i} / u_{0}, v_{j} / v_{0}$ are restrictions of rational functions $U_{i} / U_{0}, V_{j} / V_{0}$ on $R \times \mathbb{P}^{n+1} \times \mathbb{P}^{n+1}$ to $\mathscr{Z}$. The rational function field of the algebraic variety $\mathscr{X} \otimes_{R} R^{\prime}$ over $\mathbb{C}$ is $K^{\prime}\left(x_{0}, x_{1}, \cdots, x_{n}\right)$ where $x_{0}, x_{1}, \cdots, x_{n}$ are the restrictions of the coordinates on $\mathbb{A}^{n+1}$. We have shown that the map

$$
\begin{gathered}
K^{\prime}\left(x_{0}, x_{1}, \cdots, x_{n}\right) \longrightarrow K^{\prime}\left(\frac{u_{1}}{u_{0}}, \frac{u_{2}}{u_{0}}, \cdots, \frac{u_{n+1}}{u_{0}}, \frac{v_{1}}{v_{0}}, \frac{v_{2}}{v_{0}}, \cdots, \frac{v_{n+1}}{v_{0}}\right) \\
\left(x_{0}, x_{1}, \cdots, x_{n}\right) \longrightarrow\left(\frac{u_{1}}{u_{0}}, \frac{u_{2}}{u_{0}}, \cdots, \frac{u_{n+1}}{u_{0}}\right)
\end{gathered}
$$

is an isomorphism of the field. Therefore $v_{1} / v_{0}, v_{2} / v_{0}, \cdots, v_{n+1} / v_{0}$ can be expressed as quotients of polynomials in $x_{0}, x_{1}, \cdots, x_{n}$ with coefficients in $R^{\prime}$. In particular $y\left(t_{0} ; x_{0}, x_{1}, \cdots, x_{n} ; t\right), y^{(1)}\left(t_{0} ; x_{0}, x_{1}, \cdots, x_{n} ; t\right), y^{(n)}\left(t_{0} ; x_{0}, x_{1}\right.$, $\left.\cdots, x_{n} ; t\right)$ are of this type and the condition (i) is satisfied.

The proof of the Lemma shows

Corollary (3.6). The conditions (i), (ii) aand (iii) are equivalent to the following.

(iii)' There exists a point $t_{0} \in D$, an open neighbourhood $D^{\prime}$ of $t_{0}, a$ meromorphic map $\Phi: D^{\prime} \times \bar{X}_{t_{0}} \cdots \rightarrow \bar{X}^{\prime}$ making the diagram

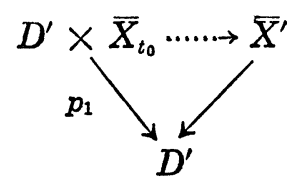

commutative such that $\Phi$ coincides with the set $Z$ of the condition (iii) on a non-empty open set of $\left(D^{\prime} \times \bar{X}_{t_{0}}\right) \times{ }_{D} \bar{X}^{\prime}$.

Corollary (3.7). Assume that the equivalent conditions (i), (ii) (iii), (iii)' are satisfied. Let $K^{\prime}$ be the quotient field of $R^{\prime}$. Then $\mathscr{Z} \otimes_{R^{\prime}} K^{\prime} \subset$ $\left.\left(X_{t_{0}} \otimes_{\mathrm{C}} R^{\prime}\right) \times_{R^{\prime}} \mathscr{X}^{\prime}\right) \otimes_{R^{\prime}} K^{\prime}=\left(X_{t_{0}} \otimes_{\mathrm{C}} R^{\prime} \otimes_{R^{\prime}} K^{\prime}\right) \times_{K^{\prime}} \mathscr{X}^{\prime} \otimes_{R^{\prime}} K^{\prime}$ defines a $K^{\prime}$. birational isomorphism of $K^{\prime}$-algebraic varieties $X_{t_{0}} \otimes_{\mathrm{C}} K^{\prime}$ and $\mathscr{X}^{\prime} \otimes_{R^{\prime}} K^{\prime}$. 
It follows from the Lemma the following Corollary which explain the geometric meaning of the Lemma.

Corollary (3.8). If the equivalent conditions of Lemma (3.5) are satisfied, then there exists a subdomain $D^{\prime}$ of $D$ such that the solution $y\left(t_{0} ; x_{0}, x_{1}, \cdots, x_{n} ; t\right)$ of the differential equation (3.2) defines birational correspondences between the fibres over $D^{\prime}$ of $X$. Namely, let $\varphi_{a, b}: X_{a} \cdots \rightarrow X_{b}$ be defined by $\varphi_{a, b}\left(x_{0}, x_{1}, \cdots, x_{n}\right)=\left(y\left(a ; x_{0}, x_{1}, \cdots, x_{n} ; b\right), y^{(1)}\left(a ; x_{0}, x_{1}, \cdots, x_{n} ; b\right)\right.$, $\cdots, y^{(n)}\left(a ; x_{0}, x_{1}, \cdots, x_{n} ; b\right) \in X_{b}$ for $x_{0}, x_{1}, \cdots, x_{n} \in X_{a}$. Then $\varphi_{a, b}$ is birational for $a, b \in D^{\prime}$.

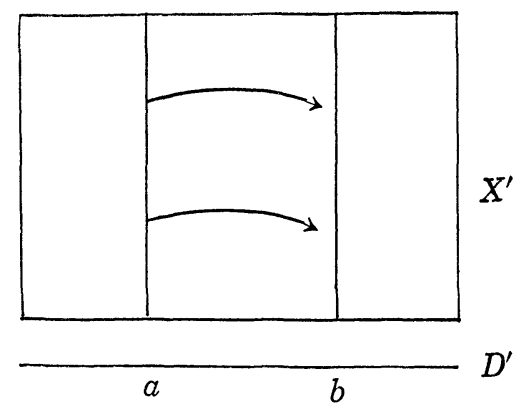

Proof. $Z$ induces bimeromorphic isomorphism $\varphi_{t_{0} t}: \bar{X}_{t_{0}} \cdots \rightarrow \bar{X}_{t}$ by (iii) for any $t \in D^{\prime}$. By G.A.G.A. $\varphi_{t_{0} t}$ is an birational isomorphism of $X_{t_{0}}$ and $X_{t}$. By the unicity of the solution of the differential equation we have

(3.8.1) $y\left(a ; x_{0}, x_{1}, \cdots, x_{n} ; b\right)=y\left(t_{0} ; \varphi_{t_{0} a}^{-1}\left(x_{0}, x_{1}, \cdots, x_{n}\right) ; b\right)$ for a general $\left(x_{0}, x_{1}, \cdots, z_{n}\right) \in X_{a}$ and the condition (ii) is satisfied.

Proposition (3.9). If the equivalent conditions (i), (ii), (iii) and (iii)' are satisfied, then for any point $t_{1} \in D^{\prime}$ and the neighbourhood $D^{\prime}$ of $t_{1}$ the conditions (i), (ii), (iii) and (iii)' are satisfied.

Proof. This is a consequence of (3.8.1) in the Proof of Corollary (3.8).

Now we can state

Definition (3.10). When the equivalent conditions of the Lemma are satisfied, we say that the general solution of the differential equation (3.2) depends rationally on the initial conditions.

It follows from Proposition (3.9), the conditions (i), (ii), (iii) and (iii)' are independent of the point $t_{0}$. Therefore we sometimes do not make the reference point $t_{0}$ clear and we can replace $D$ by any subdomain without loosing the conditions (i), (ii), (iii) and (iii)'. 
Painleve's theorem states that starting from the ring $R$ by the permissible operations of $\S 1$, we can solve the differential equation (3.2) if the general solution depends rationally on the initial condition, where we denote by $R$ the ring generated over $\mathbb{C}$ be the coefficients of the polynomial $F$ (Corollary (4.6)). Before we prove this theorem of considerable depth, we prove a special case which is a direct consequence of Theorem (2.19).

Let $X=\operatorname{Spec} \mathbb{C}\left[z_{1}, z_{2}, \cdots, z_{n}\right]$ be an affine algebraic variety over $\mathbb{C}$, $D \subset \mathbb{C}$ a domain and $K$ a field of meromorphic functions on $D$. Let $\theta \in \operatorname{Der}_{K}\left(K\left(X \otimes_{\mathrm{c}} K\right), K\left(X \otimes_{\mathrm{c}} K\right)\right)$. Then we can associate the system of Pfaffian differential equations $\operatorname{Pf}(\theta)$ on $D \times X$ as in $\S 1$. Namely let $\theta$ be given by

$$
\theta\left(z_{i}\right)=\frac{F_{i}\left(t ; z_{1}, z_{2}, \cdots, z_{n}\right)}{G_{i}\left(t ; z_{1}, z_{2}, \cdots, z_{n}\right)} \quad \text { for } 1 \leq i \leq n
$$

We consider a system of Pfaffian differential equations

$$
d z_{i}=\frac{F_{i}\left(t ; z_{1}, z_{2}, \cdots, z_{n}\right)}{G_{i}\left(t ; z_{1}, z_{2}, \cdots, z_{n}\right)} d t \quad \text { on } D \times X
$$

Replacing $D$ be a subdomain if necessary we may assume that the coefficients of the $F_{i}$ and $G_{i}$ are regular on $D$. Replacing $D$ by an appropriate subdomain, we may assume that for any $t_{0} \in D, F\left(t_{0} ; Z_{1}, Z_{2}, \cdots, Z_{n}\right)$ is not constantly equally 0 on $X$. We look for solutions of (3.11), $t \rightarrow$ $\left(t ; y_{1}(t), y_{2}(t), \cdots, y_{n}(t)\right) \in D \times X \subset D \times \mathbb{A}^{n}$, where $X \subset \mathbb{A}^{n}$ is defined by $\mathbb{C}\left[Z_{1}, Z_{2}, \cdots, Z_{n}\right] \rightarrow \mathbb{C}\left[z_{1}, z_{2}, \cdots, z_{n}\right]$ with $Z_{i} \rightarrow z_{i}$. Let $t_{0} \in D$ and $\left(z_{1}, z_{2}\right.$, $\left.\cdots, z_{n}\right) \in X$ be a non-singular point with $G_{i}\left(t_{0} ; z_{1}, z_{2}, \cdots, z_{n}\right) \neq 0$. Then there is the unique holomorphic solution $D \rightarrow D \times X, t \rightarrow\left(t ; y_{1}(t), y_{2}(t)\right.$, $\left.\cdots, y_{n}(t)\right)$, with $y_{i}\left(t_{0}\right)=z_{i}$ for $1 \leq i \leq n$. We denote $y_{i}(t)$ by $y_{i}\left(t_{0} ; z_{1}, z_{2}\right.$, $\left.\cdots, z_{n} ; t\right)$, which is holomorphic with respect to $\left(z_{1}, z_{2}, \cdots, z_{n}\right) \in X$.

LEMMA (3.12). The following conditions are equivalent.

(i) There exist a point $t_{0} \in D$, a subdomain $D^{\prime}$ of $D$ containing $t_{0}$, a non-empty open set $V$ of $X$ (for the usual topology) consisting of nonsingular points and polynomials $C_{i}\left(t ; Z_{1}, Z_{2}, \cdots, Z_{n}\right), D_{i}\left(t ; Z_{1}, Z_{2}, \cdots, Z_{n}\right)$ in $Z_{1}, Z_{2}, \cdots, Z_{n}$ with coefficients in a ring of holomorphic functions on $D^{\prime}$ such that (a) if $\left(z_{1}, z_{2}, \cdots, z_{n}\right) \in V$, then $G_{i}\left(t_{0} ; z_{1}, z_{2}, \cdots, z_{n}\right) \neq 0$ for $1 \leq i \leq n$ and such that (b) for $\left(z_{1}, z_{2}, \cdots, z_{n}\right) \in V$, 


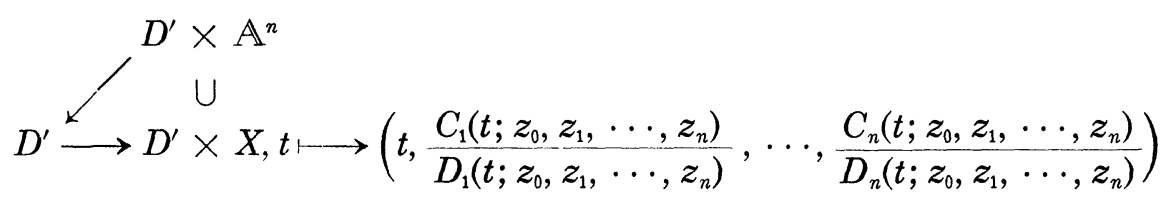

is the solution of (3.11) with

$$
\frac{C_{i}\left(t_{0} ; z_{0}, z_{1}, \cdots, z_{n}\right)}{D_{i}\left(t_{0} ; z_{0}, z_{1}, \cdots, z_{n}\right)}=z_{i}
$$

(ii) Let $\bar{X}$ be a completion of $X:$ a complete variety containing $X$ as $a$ Zariski open set. There exist a point $t_{0} \in D, a$ subdomain $D^{\prime} \subset D$ con . taining $t_{0}$ such that if we denote by $Z$ the set $\left\{\left(t ; z_{0}, z_{2}, \cdots, z_{n}, y_{1}\left(t_{0} ; z_{1}, z_{2}\right.\right.\right.$, $\left.\left.\cdots, z_{n} ; t\right), \quad y_{2}\left(t_{0} ; z_{1}, z_{2}, \cdots, z_{n} ; t\right), \cdots, y_{n}\left(t_{0} ; z_{1}, z_{2}, \cdots, z_{n} ; t\right)\right) \in D^{\prime} \times X \times_{D^{\prime}} X \mid$ $G_{i}\left(t_{0}, z_{1}, z_{2}, \cdots, z_{n}\right) \neq 0,\left(z_{1}, z_{2}, \cdots, z_{n}\right) \in X$ is a smooth point $\}$, the Zariski closure $\bar{Z} \subset\left(D^{\prime} \times \bar{X}\right) \times_{D^{\prime}}, \bar{X} \subset D^{\prime} \times \bar{X} \times \bar{X}$ defines a D-bimeromorphic correspondence of $D^{\prime} \times \bar{X}$ with itself (cf. Part I).

(iii) There exists a point $t_{0} \in D, a$ subdomain $D^{\prime} \subset D$ containing $t_{0}$ and a morphism $F: D^{\prime} \rightarrow \operatorname{Bim} X$ of functors such that $(a) F\left(t_{0}\right)=\operatorname{Id}_{X}$ and (b) $\theta=\Theta(F)$ when extended to $K\left(D^{\prime}\right)$-derivations of $K\left(D^{\prime}\right)\left(X \otimes_{\mathrm{c}} K\left(D^{\prime}\right)\right)$ (cf. $\S 1)$, where $K\left(D^{\prime}\right)$ is the field of the meromorphic functions on $D^{\prime}$.

Proof. The equivalent of (i) and (ii) is proved by the same method as in the Proof of Lemma (3.5) and hence we omit the proof. Assume now that the equivalent conditions (i) and (ii) are satisfied. We choose $t_{0}$ and $D^{\prime}$ satisfying the two conditions (i) and (ii). Then it follows from (ii) that we have a morphism $F: D^{\prime} \rightarrow \operatorname{Bim} X$ of functors. By Lemma (1.27) of Part I, we may assume that there exist an algebraic variety $Z$, a morphism $g: Z \rightarrow \operatorname{Bir} X$ of functors and a holomorphic map $f: D^{\prime} \rightarrow Z^{\text {an }}$ such that the image $f\left(D^{\prime}\right)$ is dense in the algebraic variety $Z$ and such that $F=g^{\text {an }} \circ f$. Let us use the notation of $\S 1$. Then by (ii) around $t_{0}$, $t \mapsto(t, g(t) x)$ is the solution of $\operatorname{Pf}(\theta)$. Therefore as in $\S 1 \theta=\Theta(F)$. Conversely if the condition (iii) is satisfied as we see in $\S 1$, for $x \in X_{0}$ the solution around $\left(t_{0}, x\right) \in D^{\prime} \times X$ of the system $\operatorname{Pf}(F)$ is given by $t \mapsto$ $(t, g(t) \cdot x)$. As the morphism $g$ gives a rational map $Z \times X \cdots \rightarrow$, each coordinate of the point $g(t) \cdot x$ is rational with respect to $x$ and the condition (ii) is satisfied.

Definition (3.13). When the equivalent condition (3.12) is satisfied, we say that the general solution of the system (3.11) of Pfaffian differential equations depends rationally on the initial conditions. 
By the same argument as in the Proof of Corollary (3.8), we can show that if the equivalent conditions (i), (ii) and (iii) of Lemma (3.12) are satisfied, then for any point $t_{1} \in D^{\prime}$ and the neighbourhood $D^{\prime}$ of $t_{1}$ the conditions (i), (ii) and (iii) are satisfied. Therefore we can replace $D$ by any subdomain of $D^{\prime}$ without loosing the conditions (i), (ii) and (iii).

Definition (3.14). In general for an algebraic variety $Y$ over $\mathbb{C}$ and a system of Pfaffian differential equations defined by a $\theta \in \operatorname{Der}_{K}\left(\mathbb{C}(Y) \otimes_{\mathrm{C}} K\right.$, $\left.\mathbb{C}\left(Y \otimes_{\mathrm{C}} K\right)\right)$ as in $\S 1$, we say that the general solution of the system $\operatorname{Pf}(\theta)$ of Pfaffian differential equation associated with $\theta$ depends rationally on the initial condition if there exists an affine model $X$ of $Y$ such that $\operatorname{Pf}(\theta)$ on $D \times Y$ satisfies the equivalent conditions of Lemma (3.12).

If there exists an affine model $Y$ over which $\operatorname{Pf}(\theta)$ satisfies the equivalent conditions of Lemma (3.12), then for any affine model $Z$ of $Y \operatorname{Pf}(\theta)$ satisfies the equivalent conditions of Lemma (3.12). Namely we can say that the definition is independent of the choice of affine model.

Remark (3.15.1). Assume that the equivalent conditions (i), (ii) and (iii) of Lemma (3.12) are satisfied. Then the condition (iii) gives a morphism $F(\theta): D^{\prime} \rightarrow \operatorname{Bim} \bar{X}$ of functors on the category of analytic spaces. It follows from Proposition (1.7) that denoting by $L$ the field of meromorphic functions generated over $\mathbb{C}$ by the coefficients of the polynomials $C_{i}, D_{i}$ we get the $L$-valued point $P(\theta)=P(F(\theta))$ : Spec $L \rightarrow \operatorname{Bir} \bar{X}$ or the $L$-birational automorphism of $L$-algebraic variety $X \otimes_{\mathrm{C}} L$.

(3.15.2). Suppose that the equivalent conditions (i), (ii) and (iii) of Lemma (3.12) are satisfied. We get a meromorphic map

$$
D^{\prime} \longrightarrow D^{\prime} \times X, \quad t \longmapsto\left(t, \frac{C_{1}\left(t ; z_{0}, z_{1}, \cdots, z_{n}\right)}{D_{1}\left(t ; z_{0}, z_{1}, \cdots, z_{n}\right)}, \cdots, \frac{C_{n}\left(t ; z_{0}, z_{1}, \cdots, z_{n}\right)}{D_{n}\left(t ; z_{0}, z_{1}, \cdots, z_{n}\right)}\right)
$$

Composing this with the projection $p_{2}: D^{\prime} \times X \rightarrow X$, we get a meromorphic map

$$
f: D^{\prime} \longrightarrow X, \quad t \longmapsto\left(\frac{C_{1}\left(t ; z_{0}, z_{1}, \cdots, z_{n}\right)}{D_{1}\left(t ; z_{0}, z_{1}, \cdots, z_{n}\right)}, \cdots, \frac{C_{n}\left(t ; z_{0}, z_{1}, \cdots, z_{n}\right)}{D_{n}\left(t ; z_{0}, z_{1}, \cdots, z_{n}\right)}\right)
$$

for a general $\left(z_{0}, z_{1}, \cdots, z_{n}\right)$. Replacing $D^{\prime}$ by a suitable subdomain, we may assume that $f$ is holomorphic. Then the associated point $P(f)$ to $f$ is an $L$-valued point. $P(f)$ : $\operatorname{Spec} L \rightarrow X$ is just the composite 


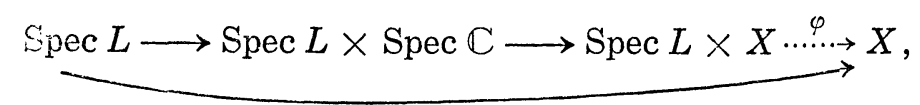

where the first map is (Id, canonical map induced by the inclusion $L \supset \mathbb{C}$ ) and the second map is (Id, Spec $\mathbb{C} \rightarrow z \in X$ ). (cf. 2).

Proposition (3.16). Let us consider the algebraic differential equation (3.2) with coefficients in a ring $R$ of holomorphic functions on $D$ such that the general solution $y\left(t_{0} ; x_{0}, x_{1}, \cdots, x_{n} ; t\right)=y(t)$ depends rationally on the initial conditions. Then there exist a field $K^{\prime} \supset \mathbb{C}$ of meromorphic functions obtained from $R$ by finite iterations of the permissible operations (O), (P1), (P2), ‥, (P5) and a K-derivation $\theta \in \mathrm{Dər}_{K}\left(K\left(X_{t_{0}} \otimes_{\mathrm{c}} K^{\prime}\right), K\left(X_{t_{0}} \otimes_{\mathrm{C}} K^{\prime}\right)\right)$ such that the general solution $t \mapsto\left(t ; v_{0}, v_{1}, \cdots, v_{n}\right)$ of the Pfaffian system $\operatorname{Pf}(\theta)$ associated with $\theta$ depends rationally on the initial condition and such that $(R[y]) P=\left(R\left[v_{0}, v_{1}, \cdots, v_{n}\right]\right) P$, where WP means the set of meromorphic functions obtained from $W$ by a finite times of iterations of the permissible operations in $\S 2$ (see Definition (2.16)).

In other words, finding a general solution of (3.2) is equivalent to finding a general solution of $\mathrm{Pf}(\theta)$ by permissible operations.

Proof. Let $K$ be the quotient field of $R$. It follows from Corollary (3.7) that there exists an extension $K^{\prime}$ of the field $K$ such that $X_{t_{0}} \otimes_{\mathrm{c}} K^{\prime}$ is birational to $\mathscr{X} \otimes_{K} K^{\prime}$. Therefore by Proposition (1.25) we may assume that $K^{\prime}$ is a finite algebraic extension of $K$. Hence $K^{\prime}$ is obtained from $R$ by finite iterations of the permissible operations (O), (P1), ., (P5). We may assume that the birational isomorphism between $X_{t_{0}} \otimes_{\mathrm{c}} K^{\prime}$ and $\mathscr{X} \otimes_{K} K^{\prime}$ is defined over a ring $R^{\prime}$ of finite type over $R$ and $R^{\prime}$ consists of holomorphic function on a subdomain $D^{\prime}$ of $D$. Taking a system of generators such that $R^{\prime}=\mathbb{C}\left[f_{1}, f_{2}, \cdots, f_{l}\right]$, we get a holomorphic map $D^{\prime} \rightarrow$ Spec $R^{\prime}$. We have a bimeromorphic correspondence $\psi: \bar{X}_{t_{0}} \times D^{\prime} \cdots \rightarrow \mathscr{X}^{\prime}$ induced by $\Psi$. Therefore the system of Pfaffian differential equations (3.3) is translated by $\psi$ on a system of Pfaffian differential equations on $\bar{X}_{t_{0}} \times D^{\prime}$. And this translation is done by rational functions in $X_{0}, X_{1}$, $\cdots, X_{n}$ with coefficients in the quotient field of $R^{\prime}$ hence permissible. Since $\psi$ commute with the projection, $\psi^{*} t=t$ and the Pfaffian system (3.3) is transformed to (3.11) and the Proposition is proved.

By Proposition (3.16), the general solution of the differential equation (3.2) whose general solution depends rationally on the initial conditions 
is reduced to that of the systems of Pfaffian differential equations (3.11) such that the coefficients of $F_{i}(t ; Z), G_{i}(t ; Z)$ are known from $R$ by permissible operations and the general solution of (3.11) depends rationally on the initial conditions.

Definition (3.17). If there exists an analytic subgroup $G$ of $\operatorname{Bim} \bar{X}$ such that the morphism $F: D \rightarrow \operatorname{Bim} \bar{X}$ factors through $G \subset \operatorname{Bim} \bar{X}$, then we say that the system $(\operatorname{Pf}(F))$ of Pfaffian differential equations is of finite type.

TheOREm (3.18). If the general solution of the system (3.11) of Pfaffian differential equations with coefficients in $R \supset \mathbb{C}$ depends rationally on the initial conditions and if the system is of finite type, then the general solution of (3.11) is in QR (i.e. there exists a non-empty Zariski open set $U$ of $X$ such that any solution with initial conditions in $U$ is in $Q R$ ), where $Q R$ is the set of meromorphic functions obtained from $R$ operating the permissible operation $Q$ for one time. In particular the general solution is obtained by a finite iteration of the permissible operations $(\mathrm{O}),(\mathrm{P} 1), \cdots,(\mathrm{P} 5)$ from $R$.

Proof. It follows from Corollary (1.33) that there exist an algebraic subgroup $H \subset \operatorname{Bir} X$ such that $\psi: D^{\prime} \rightarrow \operatorname{Bim} X$ factors through $H^{\text {an }} \rightarrow \operatorname{Bim} \bar{X}$ : $\psi\left(D^{\prime}\right) \subset H^{\text {an }} \subset \operatorname{Bim}\left(\bar{X}^{\text {an }}\right)$. Let us denote the morphism $D^{\prime} \rightarrow H^{\text {an }}$ by $\varphi$. We show that $\partial \varphi: D^{\prime} \rightarrow$ Lie $H=\mathfrak{h}$ is given by known functions (cf. Definition (2.4)). Namely let $h_{1}, h_{2}, \cdots, h_{l}$ be a basis of the Lie algebra $\mathfrak{h}$ and hence the $h_{j}^{\prime}$, are derivations at $1 \in H$. Using the notation of $\S 2$, we have $X_{\varphi(t)}=\sum_{j=1}^{l} a_{j}(\varphi(t)) R_{\varphi(t) *}\left(h_{j}\right)$ with $a_{j}(\varphi(t)) \in K$ for $t \in D^{\prime}$ where $K$ is the quotient field of $R$. In fact anyhow we can write

$$
X_{\varphi(t)}=\sum_{j=1}^{l} a_{j}(\varphi(t)) R_{\varphi(t) *}\left(h_{j}\right)
$$

with $a_{j}(\varphi(t))$ holomorphic function on $D^{\prime}$. (3.18.1) is the identity of the vector fields on the curve $\varphi\left(D^{\prime}\right)$ in $H^{\text {an }}$. Let us apply $R_{\varphi(t)-1_{*}}$ to (3.18.1) and we get

$$
\begin{aligned}
R_{\varphi(t)-1_{*}} X_{\varphi(t)} & =R_{\varphi(t)-1 *}\left(\sum_{j=1}^{l} a_{j}(\varphi(t)) R_{\varphi(t) *}\left(h_{j}\right)\right) \\
& =\sum_{j=1}^{l} a_{j}(\varphi(t)) R_{\varphi(t)-1 *} R_{\varphi(t) *}\left(h_{j}\right) \\
& =\sum_{j=1}^{h} a_{j}(\varphi(t)) h_{j} .
\end{aligned}
$$


(3.18.2) is the identity of the vector fields at $1 \in H$. Let us denote by $\iota$ : $H \rightarrow \operatorname{Bir}(\bar{X})$ the inclusion.

The morphism $\iota$ of functors on the category of $\mathbb{C}$-schemes induces $\iota_{K}$ : $H \otimes_{\mathrm{c}} K \rightarrow \operatorname{Bir}_{K}\left(\bar{X} \otimes_{\mathrm{c}} K\right)$ when we restrict the functors $H, \operatorname{Bir}(\bar{X})$ on the category of $K(\supset \mathbb{C})$-schemes. $\quad \iota_{K}$ induces a morphism $\iota_{K^{*}}:$ Lie $\left(H \otimes_{\mathrm{c}} K\right) \rightarrow$ Lie $\left(\operatorname{Bir}_{K}\left(\bar{X} \otimes_{\mathrm{C}} K\right)\right)$ of $K$-Lie algebras. We know Lie $\left(H \otimes_{\mathrm{c}} K\right)=\mathfrak{h} \otimes_{\mathrm{C}} K$, Lie $\left(\operatorname{Bir}_{K}\left(\bar{X} \otimes_{\mathrm{c}} K\right)\right)=\operatorname{Der}_{K}\left(K\left(\bar{X} \otimes_{\mathrm{c}} K\right), \quad K\left(\bar{X} \otimes_{\mathrm{c}} K\right)\right)$. Hence we get $\iota_{K^{*}}$ : $\mathscr{H} \otimes_{\mathrm{C}} K \rightarrow \operatorname{Der}_{K}\left(K\left(\bar{X} \otimes_{\mathrm{c}} K\right), K\left(\bar{X} \otimes_{\mathrm{c}} K\right)\right)$. Since $\iota_{K^{*}}$ is functional in $K$, $\iota_{K^{*}}=\iota_{\mathrm{C}^{*}} \otimes K:$ the diagram

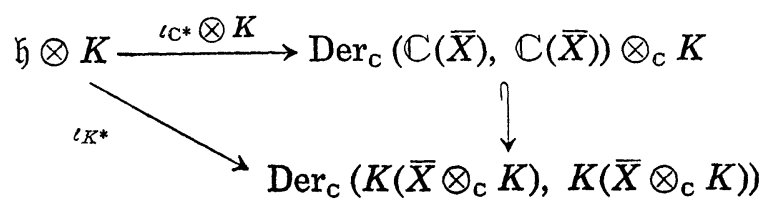

is commutative. Therefore we have

$$
\iota_{K^{*}}(h)(f) \in \mathbb{C}(\bar{X}) \text { for any } h \in \mathscr{H} \text { and } f \in \mathbb{C}(\bar{X}) .
$$

Therefore we denote $\iota_{K^{*}}$ simply by $\iota_{*}$. If we apply $\iota_{*}$ to (3.18.2), we get

$$
\begin{aligned}
\iota_{*}\left(R_{\varphi(t)-1_{*}} X_{\varphi(t)}\right)= & \iota_{*}\left[\sum_{j=1}^{h} a_{j}(\varphi(t)) h_{j}\right] \\
& =\sum_{j=1}^{l} a_{j}(\varphi(t))\left(\iota_{*}\left(h_{\jmath}\right)\right) .
\end{aligned}
$$

Recall that we have $\mathbb{C}(X)=\mathbb{C}\left(z_{0}, z_{1}, \cdots, z_{n}\right)$.

Applying the derivations in (3.18.5) to the $z_{i}$ 's, we get

$$
\iota_{*}\left(R_{\varphi(t)-1_{*}} X_{\varphi(t)}\right)\left(z_{i}\right)=\sum_{j=1}^{l} a_{j}(\varphi(t))\left(\iota^{*}\left(h_{j}\right)\right)\left(z_{i}\right)
$$

for $1 \leq i \leq n . \quad \iota_{*}\left(R_{\varphi(t)-1_{*}} X_{\varphi(t)}\right)(z)$ is in $K\left(X \otimes_{\mathrm{C}} K\right)$ hence a rational function on $X \otimes_{\mathrm{c}} K$. Let us calculate the value of the rational function $\iota_{*}\left(R_{\varphi(t)-1_{*}} X_{\varphi(t)}\right)\left(z_{i}\right)$ at a general point $z \in X \otimes_{\mathrm{c}} K$ defined over $\mathbb{C}$.

$$
\begin{aligned}
\iota_{*}\left(R_{\varphi(t)-1_{*}} X_{\varphi(t)}\right)\left(z_{i}\right)(z) & =\lim _{h \rightarrow 0} \frac{z_{i}\left(\varphi(t+h) \varphi(t)^{-1} z\right)-z_{i}(z)}{h} \\
& =\frac{F_{i}(t: z)}{G_{i}(t: z)} .
\end{aligned}
$$

For let $p: D^{\prime} \times \bar{X} \cdot \rightarrow D^{\prime} \times \bar{X},(t, z) \mapsto\left(t ;\left(A_{1}(t ; z)\right) /\left(B_{1}(t ; z)\right), \quad\left(A_{2}(t ; z)\right) /\right.$ $\left.\left(B_{2}(t ; z)\right), \cdots,\left(A_{n}(t ; z)\right) /\left(B_{n}(t ; z)\right)\right)$ be the birational transformation defined by the solution of (3.11) such that $A_{i}\left(t ; Z_{1}, Z_{2}, \cdots, Z_{n}\right)=A_{i}(t ; Z), B_{i}\left(t ; Z_{1}\right.$, 
$\left.Z_{2}, \cdots, Z_{n}\right)=B_{i}(t ; Z)$ are polynomials with holomorphic coefficients as above. Let us briefly denote $\left(A_{i}(t, z)\right) /\left(B_{i}(t, z)\right)=C_{i}(t ; z), 1 \leq i \leq m$ or more simply $(t, z) \mapsto\left(t ; C_{1}(t ; z), C_{2}(t ; z), \cdots, C_{m}(t ; z)\right)=(t ; C(t ; z))$. Since by Proposition (1.7) this correspondence is $M$-birational automorphism of $X \otimes_{\mathrm{c}} M$ where $M$ is the extension of $K$ by the adjunction of the coefficients of $A_{i}$ and $B_{i}, 1 \leq i \leq n$. We have the inverse transformation $q: D^{\prime} \times \bar{X} \cdot \rightarrow D^{\prime} \times \bar{X}$, $(t, z) \mapsto\left(t ; D_{1}(t ; z), D_{2}(t ; z), \cdots, D_{n}(t ; z)\right)=(t, D(t ; z))$ so that $C(t ; D(t ; x))=$ $x, D(t ; C(t ; x))=x$. We choose a point $s \in D$ and fix it.

On the one hand since $t \mapsto(t ; C(t ; z)$ is a solution, we have

$$
d C_{i}\left(t ; z^{\prime}\right)=\frac{F_{i}\left(t ; C\left(t ; z^{\prime}\right)\right)}{G_{i}\left(t ; C\left(t ; z^{\prime}\right)\right)} d t
$$

for $1 \leq i \leq n$ and for a general point $z^{\prime}$ of $\bar{X}$. We can substitute $D(s ; z)$ for $z^{\prime}$. Then (3.18.7) becomes

$$
d C_{i}(t ; D(s ; z))=\frac{F_{\imath}(t ; D(s ; z))}{G_{i}(t ; D(s ; z))} d t
$$

Therefore at $t=s$

$$
\left.\frac{d C_{i}(t ; D(s ; z))}{d t}\right|_{t=s}=\frac{F_{i}(s ; z)}{G_{i}(s ; z)} .
$$

This is what we had to prove.

We have thus proved the commutativity of the following diagram:

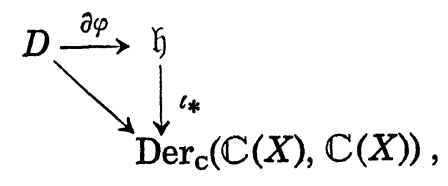

where the map $D \rightarrow \operatorname{Der}_{\mathrm{C}}(\mathbb{C}(X), \mathbb{C}(X))$ is given by $t \mapsto \sum_{i=1}^{n}\left(F_{i}(t ; z)\right) /\left(G_{i}(t ; z)\right)$ $\left(\partial / \partial Z_{i}\right)$. Next we want to show that $\tau(\varphi) \in \mathfrak{h} \otimes K$ (cf. Lemma (2.13)). Let $F$ be an extension of the field $M$ such that $\tau(\varphi) \in \mathfrak{h} \otimes M$. It follows from (3.18.10) that $\iota_{*} \otimes_{\mathrm{c}} F(\tau(\varphi)) \in \operatorname{Der}_{\mathrm{c}}(\mathbb{C}(X), \mathbb{C}(X)) \otimes F$ is in the image of $\operatorname{Der}_{\mathrm{C}}(\mathbb{C}(X), \mathbb{C}(X)) \otimes K$. Since $\iota_{*}$ is injective, $\tau(\varphi) \in \mathfrak{h} \otimes K$ by the following sublemma.

Sublemma (3.18.11). Let $k$ be a field $V_{1}, V_{2}$ be k-vector spaces and $W_{1} \subset V_{1}, W_{2} \subset V_{2}$ be subspaces. Then

$$
W_{1} \otimes_{k} W_{2}=W_{1} \otimes_{k} V_{2} \cap V_{1} \otimes W_{2},
$$


where the vector spaces above are identified with their canonical images in $V_{1} \otimes V_{2}$.

Proof. Since the inclusion $\subset$ is evident, it is sufficient to show $\supset$. Let $x \in W_{1} \otimes_{k} V_{2} \cap V_{1} \otimes W_{2}$. Then $x=\sum_{i=i}^{s} a_{i} \otimes b_{i}, x=\sum_{j=1}^{t} c_{j} \otimes d_{j}, a_{i} \in W_{1}$, $b_{i} \in V_{2}, c_{j} \in V_{1}, d_{j} \in W_{2}$. Therefore we may assume that $V_{1}, V_{2}$ are of finite dimension. The Sublemma is proved by taking a basis $e_{1}, e_{2}, \cdots, e_{r_{1}}, e_{r_{1}+1}$, $\cdots, e_{r}$ (resp. $f_{1}, f_{2}, \cdots, f_{s_{1}}, f_{s_{1}+1}, \cdots, f_{s}$ ) of $V_{1}$ (resp. of $V_{2}$ ) such that $e_{1}, e_{2}$, $\cdots, e_{r_{1}}$ (resp. $f_{1}, f_{2}, \cdots, f_{s_{1}}$ ) span $W_{1}$ (resp. $W_{2}$ ).

Therefore $\partial \psi: D^{\prime} \rightarrow$ Lie $H=\mathfrak{h}$ is written using known functions from $K$ by the operation $Q$. Therefore for any rational function $f$ on $H, f \circ \varphi$ is a new known function by the permissible operation $Q$. It follows from Proposition (1.6) of Part I that the inclusion $H \subset \operatorname{Bir} X$ is given by an algebraic pseudo-operation $(H, \rho, X)$. For a general point $z \in X$, we denote by $H_{z}$ the Zariski closure $\overline{\{\varphi((h, z)) \mid h \in H, \rho: H \times X \cdots X \text { is regular at }(h, z)\}}$. We have thus a dominant rational map $p: H \rightarrow H_{z}$. For a general $z, t \mapsto$ $(t, \varphi(t) z)$ is the solution of (3.11) with initial condition $\varphi\left(t_{0}\right) z=z$. We consider the composite rational map $H \stackrel{p}{\rightarrow} H_{x} \stackrel{z_{i}}{\rightarrow} \mathbb{A}^{1}$. Therefore $\left(z_{i} \circ p\right) \circ \varphi(t)$ $=\left(A_{i}(t ; z)\right) /\left(B_{i}(t ; z)\right)$ is a known function by the permissible operation $Q$ applied for $R, \partial \psi$ and $H$. The last assertion follows from Theorem (2.19).

Remark (3.19). In the last part of the proof, we can argue in an intrisic manner. In fact, let $N$ be the field obtained from $R$ by the operation $Q$ with respect to $\partial \psi$ and $H$. It is sufficient to show that the point $P(y(z)) \in X$ associated with the general solution $y(z): D^{\prime} \rightarrow D^{\prime} \times X, t \mapsto$ $\left(t ; C_{1}(t ; z) / D_{1}(t ; z), \cdots, C_{n}(t ; z) / D_{n}(t ; z)\right)$ is $N$-rational. The point $P(\varphi) \in H$ associated with $\varphi: D^{\prime} \rightarrow H$ is $N$-rational and $z \in X$ is $\mathbb{C}$-rational in particular $N$-rational. Therefore $P(\varphi) z \in X$ is $N$-rational by $\S 1$. We have further $P(\varphi) z=P(y(z))$.

Definition (3.20). Let $D \subset \mathbb{C}$ be a domain, $R$ a ring of holomorphic functions and $K$ the quotient field of $R$. Let $X$ be an algebraic variety over $\mathbb{C}$. Let $\theta \in \operatorname{Der}_{K}\left(K\left(X \otimes_{\mathrm{c}} K\right), K\left(X \otimes_{\mathrm{c}} K\right)\right)$. For any algebraic variety $Y$ birationally equivalent to $X, \theta$ defines the system of Pfaffian differential equations on $D \times Y$. If there exists a complete, non-singular model $Y$ of $X$ over $\mathbb{C}$ such that $\theta$ is a regular vector field of $Y \otimes_{\mathrm{c}} K$, then we say that we can compactify the space of initial conditions of $\operatorname{Pf}(\theta)$ on $D \times X$.

In the language of differential equation, if we can compactify the space of initial conditions, there exists a subdomain $D^{\prime}$ such that $\operatorname{Pf}(\theta)$ defines 
a regular system of Pfaffian differential of corank 1 on $D^{\prime} \times Y$.

Theorem (3.21). The system of Pfaffian differential equations $\operatorname{Pf}(\theta)$ on $D \times Y$ is of finite type if and only if we can compactify the space of initial conditions.

Proof. Assume that the system is of finite type, let $\psi: D \rightarrow \operatorname{Bim}(\bar{X})$ factor through an algebraic group $\varphi: D \rightarrow H \subset \operatorname{Bir} X$ and $H \subset \operatorname{Bir} X$ is defined by an algebraic pseudo-operation $(H, \rho, X)$. Then by [U2], we can find a non-singular projective model $Y$ of $X$ such that $H$ operates regularly on $Y$. Now the solutions of $\operatorname{Pf}(\theta)$ on $D \times Y$ are orbits $\{\varphi(t) \cdot z \mid z \in Y\}$ and the vector field $\theta$ on $D \times Y$ is given as in $\S 1$. Hence $\theta=\Theta(\varphi,(G, Y))$ and regular on $Y \otimes_{c} K$. Conversely if we can compactify the space of initial conditions, then by G.A.G.A. [G1] the solution depends rationally on the initial conditions and $\psi^{\prime}: D \rightarrow \operatorname{Bim} Y$ factors through $\operatorname{Aut}^{\circ} Y \subset \operatorname{Bir} Y$ and $\operatorname{Aut}^{\circ} Y$ is an algebraic group ([G2]).

\section{\$4. Solbavility theorem of Painlevé (general case)}

We prove a more general solvability theorem than in $\S 3$. Some special cases of the theorem was proved by Painlevé and Picard (cf. Painlevé $[\mathrm{P}]$, p. 393). Let us begin by some preliminary results on differential fields.

We consider ordinary differential rings (i.e. commutative rings with a differentiation) of characteristic 0 such as rings of meromorphic functions on a domain of $\mathbb{C}$ with the usual differentiation. We often denote by $y^{\prime}, y^{\prime \prime}, \cdots, y^{(l)}$ the differentiations of $y, y^{\prime}, \cdots, y^{(l-1)}$. Let $K$ be a differential field, $k$ a differential subfield of $K$ and $y_{1}, y_{2}, \cdots, y_{n} \in K$. Then we denote by $k\left\{y_{1}, y_{2}, \cdots, y_{n}\right\}$ the differential subring of $K$ generated over $k$ by $y_{1}, y_{2}$, $\cdots, y_{n}$ : the $k$-algebra generated by the $y_{i}$ and their derivations $D^{j}\left(y_{i}\right)$ over $k(1 \leqq i \leqq n, 1 \leqq j)$. We denote by $k\left\langle y_{1}, y_{2}, \cdots, y_{n}\right\rangle$ the differential subfield of $K$ generated over $k$ by $y_{1}, y_{2}, \cdots, y_{n}$.

Our differential rings are considered in a big differential field, which often we do not mention if there is no danger of confusion.

Let $k$ be a differential field and the $Y_{i j}(1 \leqq i \leqq n, 1 \leqq j)$ variables over $k$. Then extending the derivation $D$ of $k$ by $D\left(Y_{i j}\right)=D\left(Y_{i j+1}\right)$, we get a differential ring $k\left[Y_{i j}\right]_{1 \leqq i \leqq n, 1 \leqq j}$ which we denote by $k\left\{Y_{1}, Y_{2}, \cdots, Y_{n}\right\}$. We call $k\left\{Y_{1}, Y_{2}, \cdots, Y_{n}\right\}$ the differential polynomial ring of $n$-variables over $k$. The quotient field of $k\left\{Y_{1}, Y_{2}, \cdots, Y_{n}\right\}$ is denoted by $k\left\langle Y_{1}, Y_{2}, \cdots, Y_{n}\right\rangle$. Let 
$S$ be a set of elements of a differential ring $A$. The differential ideal (ideal of $A$ closed under the differentiation) generated by $S$ is denoted by [S]. If $I$ is a differential ideal, then $A / I$ is a differential ring. We recall the following well-known fact.

Lemma (4.1). Let $k$ be a differential field. Let $k\langle y\rangle$ be an extension. Let $F(Y) \in k\{Y\}$ be a non-zero polynomial with $F\left(y, y^{\prime}, y^{\prime \prime}, \cdots, y^{(l)}\right)=0$ of minimum order (there is no non-zero polynomial $G\left(Y, Y_{1}, Y_{2}, \cdots, Y_{l_{-1}}\right) \in k\{Y\}$ such that $G\left(y, y^{\prime}, y^{\prime \prime}, \cdots, y^{(l-1)}\right)=0$ and of minimum degree in $Y^{(l)}$. Then the ring $k\langle y\rangle$ is $k$-isomorphic to the quotient field of the localization $(k\{Y\} \mid$ $[F])_{H}$ where $H=\partial F / \partial Y_{l}$.

Proof. It follows from $F\left(y, y^{\prime}, y^{\prime \prime}, \cdots, y^{(l)}\right)=0$ that $y^{(l)}$ is algebraic over $k\left(y, y^{\prime}, y^{\prime \prime}, \cdots, y^{(l-1}\right)$. Let

$$
F\left(y, y^{\prime}, \cdots, y^{(l)}\right)=a_{0}\left(y^{(l)}\right)^{n}+a_{1}\left(y^{(l)}\right)^{n-1}+\cdots+a_{n}
$$

with $a_{i} \in k\left[y, y^{\prime}, \cdots, y^{(l-1)}\right], a_{0} \neq 0$. Then $y, y^{\prime}, \cdots, y^{(l-1)}$ are transcendental over $k$ and the field $k\left(y, y^{\prime}, \cdots, y^{(l)}\right)$ is $k$-isomorphic to $k\left(Y, Y_{1}, \cdots, Y_{l-1}\right)[Z] /$ $\left(a_{0} Z^{n}+a_{1} Z^{n-1}+\cdots+a_{n}\right)$ by the choice of $F$. Differentiating (4.1.1), we get

$$
a_{0}^{\prime}\left(y^{(l)}\right)^{n}+a_{1}^{\prime}\left(y^{(l)}\right)^{n-1}+\cdots+a_{n}^{\prime}+H\left(y, y^{\prime}, \cdots, y^{(l)}\right) y^{(l+1)}=0 .
$$

By the choice of $F, n a_{0}\left(y^{(l)}\right)^{n-1}+(n-1) a_{1}\left(y^{(l)}\right)^{n-2}+\cdots+a_{n-1} \neq 0$ and hence $y^{(l+1)}$ is in $k\left(y, y^{\prime}, \cdots, y^{(l)}\right)$. The above isomorphism gives the disered isomorphism (cf. Exercise 1, (b), p. 163, [Ko]).

Lemma (4.2). Let $a_{1}(t), a_{2}(t), \cdots, a_{l}(t), b_{1}(t), b_{2}(t), \cdots, b_{m}(t), c_{1}(t), c_{2}(t), \cdots$, $c_{n}(t)$ be homomorphic functions on a domain $D \subset \mathbb{C}(l, n \geqq 0, m \geqq 1)$. If one of the $b_{i}$ is transcendental over $\mathbb{C}\left\langle a_{1}, a_{2}, \cdots, a_{l}\right\rangle\left(b_{i}\right.$ satisfies no nontrivial algebraic equations with coefficients in $\left.\mathbb{C}\left\langle a_{1}, a_{2}, \cdots, a_{l}\right\rangle\right)$, then there exist a subdomain $D^{\prime}$ of $D$, a domain $D_{1} \subset \mathbb{C}$, a point $s_{0} \in D_{1}$ and holomorphic functions $B_{i}(t, s), C_{j}(t, s)(1 \leqq i \leqq m, 1 \leqq j \leqq n)$ such that $(1) b_{i}(t)$ $=B_{i}\left(t, s_{0}\right)(1 \leqq i \leqq m), c_{j}(t)=C_{j}\left(t, s_{0}\right)(1 \leqq j \leqq n)$ for $t \in D^{\prime}$, (2) at least one of the $B_{i}(t, s)$ depends effectively on $s$ or $\partial B_{i} /\left.\partial s\right|_{s=s_{0}} \neq 0$ and such that we have an isomorphism of $\mathbb{C}\left\{a_{1}(t), a_{2}(t), \cdots, a_{l}(t)\right\}$-differential algebrais $\left(\mathbb{C}\left\{a_{1}(t)\right.\right.$, $\left.\left.a_{2}(t), \cdots, a_{l}(t), b_{1}(t), b_{2}(t), \cdots, b_{m}(t), c_{1}(t), c_{2}(t), \cdots, c_{n}(t)\right\}, d / d t\right) \widetilde{(}\left(\mathbb{C}\left\{a_{1}(t), a_{2}(t)\right.\right.$, $\left.\cdots, a_{\imath}(t), B_{1}(t, s), B_{2}(t, s), \cdots, B_{m}(t, s), C_{1}(t, s), C_{2}(t, s), \cdots, C_{n}(t, s), \partial / \partial t\right)$ by $b_{i}(t)$ $\mapsto B_{i}(t, s), c_{\jmath}(t) \mapsto C_{\jmath}(t, s)$.

Proof. Let $b_{k}$ be transcendental over $\mathbb{C}\left\{a_{1}, a_{2}, \cdots, a_{l}\right\}$ with minimum $k$. By enlarging $\left\{a_{1}, a_{2}, \cdots, a_{l}\right\}$ to $\left\{a_{1}, a_{2}, \cdots, a_{l}, b_{1}, \cdots, b_{k-1}\right\}$ and $\left\{c_{1}, c_{2}, \cdots, c_{n}\right\}$ 
to $\left\{b_{k+1}, b_{k+2}, \cdots, b_{m}, c_{1} \cdots, c_{n}\right\}$, we may assume that $m=1$. We denote $b_{1}$ by $b$. First we treat the case $n=0$. We put $k=\mathbb{C}\left\langle a_{1}, a_{2}, \cdots, a_{l}\right\rangle$. If $b$ satisfies no algebraic differential equations with coefficients in $k$ or $b, b^{\prime}, b^{\prime \prime}, \cdots$ are algebraically independent over $k$, then we take $D^{\prime}=D, D_{1}=\mathbb{C} s_{0}=0$ and $B(t, s)=s+b(t)$. Therefore let us assume that $b$ satisfies an algebraic differential equation. Let $0 \neq F(Y) \in \mathbb{C}\left\{a_{1}, a_{2}, \cdots, a_{\imath}\right\}\{Y\}$ with $F\left(b, b^{\prime}, \cdots\right.$, $\left.b^{(p)}\right)=0$ of minimal order $p$ and of minimal degree in $Y_{p}$. Let $F\left(Y, Y_{1}, \cdots, Y_{p}\right)$ $=A_{0}\left(Y_{p}\right)^{N}+A_{1} Y_{p}^{N-1}+\cdots+A_{N}$ with $A_{i} \in \mathbb{C}\left\{a_{1}, a_{2}, \cdots, a_{t}\right\}\left[Y, Y_{1}, \cdots, Y_{p-1}\right]$ $0 \leqq i \leqq N, A_{0} \neq 0$. It follows from the choice of $F$ that $\left(\partial F / \partial Y_{p}\right)\left(b, b^{\prime}, \cdots\right.$, $\left.b^{(p-1)}\right) \neq 0$ hence there exists a point $t_{0} \in D$ such that $\left.\left(\partial F / \partial Y_{p}\right)\left(b, b^{\prime}, \cdots, b^{(p)}\right)\right|_{t=t_{0}}$ $\neq 0$. Let $\beta_{0}=b\left(t_{0}\right), \beta_{1}=b^{\prime}\left(t_{0}\right), \cdots, \beta_{p-1}=b^{(p-1)}\left(t_{0}\right)$. Then there exist an open neighbourhood $U$ of $\left(\beta_{0}, \beta_{1}, \cdots, \beta_{p-1}\right) \in \mathbb{C}^{p}$ an open neighbourhood $D^{\prime}$ of $t_{0}$ and a holomorphic function $G\left(t, Z_{0}, Z_{1}, \cdots, Z_{p-1}\right)$ on $D^{\prime} \times U$ such that the differential equation $F\left(y, y^{\prime}, \cdots, y^{(p)}\right)=0$ with initial condition $\left(y\left(t_{0}\right), y^{\prime}\left(t_{0}\right)\right.$, $\left.\cdots, y^{(p-1)}\left(t_{0}\right)\right) \in U$ is equivalent to $y^{(p)}=G\left(t, y, y^{\prime}, \cdots, y^{(p-1)}\right)$. Therefore we can parametrize the solutions by the initial conditions at $t_{0}$. There are thus $p \geqq 1$ parameters. In particular replacing $D^{\prime}$ by a smaller open set we can find a domain $D_{1} \subset \mathbb{C}$ and a holomorphic function $B(t, s)$ on $D^{\prime} \times D_{1}$ such that (1) $\partial B / \partial s \neq 0, F\left(B, \partial B / \partial t, \cdots, \partial^{p} B / \partial t^{p}\right)=0$. Therefore we have $k$-morphism of differential algebras $\varphi:(k\{Y\} /[F])_{H} \rightarrow\left(\mathbb{C}\left\langle a_{1}, a_{2}, \cdots, a_{l}\right\rangle\{B\}\right.$, $\partial / \partial t), \quad Y \mapsto B(t, s) . \quad \varphi$ is injective since specialization at $s=s_{0}, \quad Y \mapsto B\left(t, s_{0}\right)$ is injective by Lemma (4.1). We have therefore a $k$-isomorphism of the quotient field of $(k\{Y\} /[F])_{H}$ onto $\left(\mathbb{C}\left\langle a_{1}, a_{2}, \cdots, a_{l}, B\right\rangle, \partial / \partial t\right)$. It follows from Lemma (4.1) that we have an injective $k$-morphism of $\mathbb{C}\left\{a_{1}, a_{2}, \cdots, a_{l}, b\right\}$ $\rightarrow k\langle b\rangle \widetilde{a}$ quotient field of $(k\{Y\} /[F])_{H} \widetilde{C}\left\langle a_{1}, a_{2}, \cdots, a_{l}, B\right\rangle$ inducing iso-

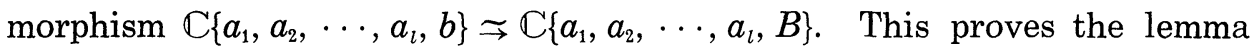
for $n=0$. Let us assume that we have a $k$-isomorphism $\varphi: \mathbb{C}\left\{a_{1}, a_{2}, \cdots\right.$, $\left.a_{l}, b\right\} \rightarrow \mathbb{C}\left\{a_{1}, a_{2}, \cdots, a_{l}, B\right\}$. We want to find new $D^{\prime}, D_{1}$ and a holomorphic function $C_{1}$ to extend $\varphi$ to an isomorphism $\mathbb{C}\left\{a_{1}, a_{2}, \cdots, a_{l}, b, c_{1}\right\} \rightarrow \mathbb{C}\left\{a_{1}, a_{2}\right.$, $\left.\cdots, a_{\imath}, B, C_{1}\right\}$. The case where $c_{1}$ satisfies no algebraic differential equation with coefficients in $k\langle b\rangle$ is the easiest. We may use $D^{\prime}, D_{1}$ for $b$ and $C(t, s)=c(t)$. The next extreme case is $c_{1}$ is algebraic over $k\{b\}$. Let $F=0$ be a defining equation of $c_{1}$ over $\mathbb{C}\left\{a_{1}, a_{2}, \cdots, a_{l}, b\right\}$. Let $\bar{F}$ be a corresponding polynomial with coefficients in $\mathbb{C}\left\{a_{1}, a_{2}, \cdots, a_{l}, B\right\}$. Choosing sufficiently small new $D^{\prime}$ and $D$ so that the algebroid function of two variables $(t, s)$ with $\bar{F}\left(C_{1}\right)=0$ is one valued, we can construct differential isomorphism $\mathbb{C}\left\{a_{1}, a_{2}, \cdots, a_{l}, b, c_{1}\right\} \widetilde{C}\left\{a_{1}, a_{2}, \cdots, a_{l}, B, C_{1}\right\}$. Since the re- 


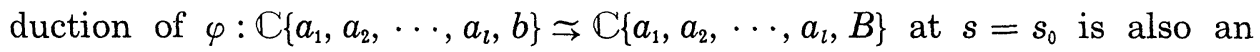
isomorphism, we may assume that $s_{0} \in D$ and $C_{1}\left(t, s_{0}\right)=c_{1}(t)$. If $c_{1}$ is not algebraic over $k\{b\}$ and satisfies an algebraic differential equation over $k\{b\}$, let $0 \neq F(Y) \in \mathbb{C}\left\{a_{1}, a_{2}, \cdots, a_{n}, b\right\}\{Y\}$ be a polynomial with $F\left(c_{1}, c_{1}^{\prime}, c_{1}^{\prime \prime}\right.$, $\left.\cdots, c_{1}^{(p)}\right)=0$ of minimum order $p \geqq 1$ and of minimum degree in $Y_{p}$. Let $\mathscr{F}(Y) \in \mathbb{C}\left\{a_{1}, a_{2}, \cdots, a_{n}, B\right\}\{Y\}$ be the polynomial obtained from $F$ by the isomorphism $\mathbb{C}\left\{a_{1}, a_{2}, \cdots, a_{n}, b\right\} \simeq \mathbb{C}\left\{a_{1}, a_{2}, \cdots, a_{a}, B\right\}$. Let $D^{\prime}, D_{1}$ and $t_{0}$ be chosen when we determined $B$. Let $F(Y)=A_{0} Y_{p}^{N}+A_{1} Y_{p}^{N-1}+\cdots+A_{N}$ with $A_{0} \neq 0, A_{i} \in \mathbb{C}\left\{a_{1}, a_{2}, \cdots, a_{l}, b\right\}\left[Y, Y_{1}, Y_{2}, \cdots, Y_{p-1}\right]$. By the choice of $F,\left(\partial F / \partial Y_{p}\right)\left(c_{1}, c_{1}^{\prime}, c_{1}^{\prime \prime}, \cdots, c_{1}^{(p)}\right) \neq 0$. Therefore there exist $t_{1} \in D^{\prime}$ such that $\left.\left(\partial F / \partial Y_{p}\right)\left(c_{1}, c_{1}^{\prime}, c_{1}^{\prime \prime}, \cdots, c_{1}^{(p)}\right)\right|_{t=t_{1}} \neq 0$. Let $A_{i}=A_{i}\left(a ; b ; Y, Y_{1}, Y_{2}, \cdots, Y_{p-1}\right)=$ $A_{i}\left(a_{1}, a_{2}, \cdots, a_{l} ; b ; Y_{1}, Y_{2}, \cdots, Y_{p-1}\right)$. Then

$$
\mathscr{F}(Y)=\tilde{A}_{0} Y_{p}^{N}+\tilde{A}_{1} Y_{p}^{N-1}+\cdots+\tilde{A}_{N},
$$

where $\tilde{A}_{i}=A_{i}\left(a ; b ; Y, Y_{1}, Y_{2}, \cdots, Y_{p-1}\right)$ which are polynomials with holomorphic coefficients on $D^{\prime} \times D_{1}$. Since $B\left(t, s_{0}\right)=b(t),\left(\partial \mathscr{F} / \partial Y_{p}\right)\left(a ; B ; c_{1}, c_{1}^{\prime}\right.$, $\left.\cdots, c_{1}^{(p)}\right)\left.\right|_{t=t_{1}, s=s_{0}} \neq 0$, there exists a holomorphic function $\mathscr{H}\left(t, s ; z_{0}, z_{1}, \cdots\right.$, $\left.z_{p-1}\right)$ regular on a neighbourhood of $\left(t_{1}, s_{0} ; c_{1}\left(t_{1}\right), c_{1}^{\prime}\left(t_{1}\right), \cdots, c_{1}^{(p-1)}\left(t_{1}\right)\right) \in \mathbb{C}^{p+2}$ such that the differential equation (4.2.1) is equivalent to a partial differential equation

$$
\frac{\partial^{p} C(t, s)}{\partial t^{p}}=\mathscr{H}\left(t, s ; c, \frac{\partial c}{\partial t}, \cdots, \frac{\partial^{p-1} c}{\partial t^{p-1}}\right)
$$

near $\left(t_{1}, s_{0} ; c_{1}\left(t_{1}\right), c_{1}^{\prime}\left(t_{1}\right), \cdots, c_{1}^{(p-1)}\left(t_{1}\right)\right)$. And $c_{1}(t)$ is a holomorphic solution of $E_{s_{0}}$ with initial conditions at $t_{1}\left(c_{1}\left(t_{1}\right), c_{1}^{\prime}\left(t_{1}\right), \cdots, c_{1}^{(p-1)}\left(t_{1}\right)\right)$. Therefore $c_{1}(t)$ can be extended to a holomorphic solution $C_{1}(t, s)$ of $E_{s}$ with $\left(C_{1}\left(t_{1}, s_{0}\right), \cdots\right.$, $\left.\left(\partial^{p-1} C_{p-1} / \partial t^{p-1}\right)\left(t_{1}, s_{0}\right)\right)=\left(c_{1}\left(t_{1}\right), c_{1}^{\prime}\left(t_{1}\right), \cdots, c_{1}^{(p-1)}\left(t_{1}\right)\right), C_{1}\left(t, s_{0}\right)=c_{1}(t)$. The same argument as above shows that we have an isomorphism $\mathbb{C}\left\{a_{1}, a_{2}, \cdots, a_{l}, b, c_{1}\right\}$ $\widetilde{C}\left\{a_{1}, a_{2}, \cdots, a_{l}, B, C_{1}\right\}$. Repeating this argument, we can construct $\mathbb{C}\left\{a_{1}, a_{2}, \cdots, a_{l}, b, c_{1}, \cdots, c_{n}\right\} \simeq \mathbb{C}\left\{a_{1}, a_{2}, \cdots, a_{l}, B, C_{1}, C_{2}, \cdots, C_{n}\right\}$ required in Lemma.

More generally we can prove the following theorem. The theorem was proved by Painlevé ([P], pp. 368-373) when $X$ is the 2-dimensional affine space.

THEOREM (4.3). We keep the notation of $\S 3$. If the general solution of the system (3.11) of Pfaffian differential equations with coefficients in a ring $R$ depends rationally on the initial conditions. Then the general solution is obtained from $R$ by the permissible operations (O), (P1), (P2), , , (P5). 
Proof. Let us first treat the case $n=1$. Let $\tilde{X}$ be a non-singular projective model of $X$. Then it follows from Corollary (1.30) of Part I that $D \rightarrow \operatorname{Bim} \tilde{X}$ factors through algebraic group Aut $^{0} \tilde{X} \subset \operatorname{Bir} X$. Therefore the system (3.11) of Pfaffian differential equations is of finite type and the theorem follows from Theorem (3.18).

Now we show the theorem for $X$ rational and next for $X$ with irregularity $q(\bar{X})=0$, where $\bar{X}$ is a non-singular projective model of $X$ and $q(\bar{X})=\operatorname{dim} H^{1}\left(\bar{X}, O_{X}\right)$ which is a birational invariant of $X$. The last step is to explain how we can treat the general case.

So let us assume first that $X$ is rational. We may assume that $X=\mathbb{A}^{n}$ so that $\mathbb{C}(X)=\mathbb{C}\left(z_{1}, z_{2}, \cdots, z_{n}\right)$. As the following argument shows, it is enough to treat the case $n=2$. The idea of the following proof is due to Painlevé $[\mathrm{P}]$. Let $D \rightarrow D \times \mathbb{A}^{2}$,

$$
t \mapsto\left(t, \frac{A_{1}(t ; x)}{B_{1}(t ; x)}, \frac{A_{2}(t ; x)}{B_{2}(t ; x)}\right)
$$

with $x=\left(x_{1}, x_{2}\right) \in \mathbb{A}^{2}=\mathbb{C}^{2}$ be the general solution as in Lemma (3.12). Namely $A_{i}(t ; Z), B_{i}(t ; Z)$ are polynomials of two variables $Z_{1}, Z_{2}$ with holomorphic coefficients. Let $K$ be the quotient field of $R$ and $L$ be the extension of $K$ generated by the coefficients of the polynomials $A_{1}, A_{2}$, $B_{1}, B_{2}$. Then our assumption implies that

$$
\left(x_{1}, x_{2}\right) \mapsto\left(\frac{A_{1}\left(t ; x_{1}, x_{2}\right)}{B_{1}\left(t ; x_{1}, x_{2}\right)}, \frac{A_{2}\left(t ; x_{1}, x_{2}\right)}{B_{2}\left(t ; x_{1}, x_{2}\right)}\right)
$$

defines a birational automorphism of $\mathbb{A}_{L}^{2}$ (see Lemma (3.12) and Proposition (1.7)).

Let $\left(u_{1}, u_{2}\right) \mapsto\left(\frac{C_{1}\left(t ; u_{1}, u_{2}\right)}{D_{1}\left(t ; u_{1}, u_{2}\right)}, \frac{C_{2}\left(t ; u_{1}, u_{2}\right)}{D_{2}\left(t ; u_{1}, u_{2}\right)}\right)$ with $C_{i}\left(t, U_{1}, U_{2}\right), D_{i}\left(t, U_{1}, U_{2}\right)$ $\in L\left[U_{1}, U_{2}\right]$ be the inverse transformation. We take $C_{i}$ and $D_{i}$ are relatively prime in $L\left[U_{1}, U_{2}\right]$. We have to show that each coefficients of $A_{1}, A_{2}, B_{1}, B_{2}$ are in $R Q$. To this end it is sufficient to show that each coefficients of $C_{1}, C_{2}, D_{1}, D_{2}$ is in $R Q$ : namely the birational automorphism (4.3.2) of $A_{L}^{2}$ is defined over a subfield of $R Q$. By Lemma (1.26) we can find $a_{i}, b_{i}, c_{i} \in \mathbb{C}(1 \leqq i \leqq 3)$ such that $\operatorname{det}\left|\begin{array}{lll}a_{1} & b_{1} & c_{1} \\ a_{2} & b_{2} & c_{2} \\ a_{3} & b_{3} & c_{3}\end{array}\right| \neq 0$ and such that the polynomials $a_{i} D+b_{i} D C_{1} / D_{1}+c_{i} D C_{2} / D_{2}$ are absolutely irreducible, where $D=$ l.c.m. $\left(D_{1}, D_{2}\right)$ in $L\left[U_{1}, U_{2}\right]$. Thus we can choose a homogeneous coordinate system on $\mathbb{P}_{\mathrm{c}}^{2}$ such that $C_{1}, C_{2}, D_{1}, D_{2}$ are absolutely irreducible 
(i.e. irreducible) in $\bar{L}\left[U_{1}, U_{2}\right]$ ) and $D_{1}=D_{2}$. From now on we assume that $C_{1}, C_{2}, D_{1}, D_{2}$ are absolutely irreducible and $D_{1}=D_{2}=E$. Let us put to simplify the notation $f_{i}(t ; x)=\left(A_{i}(t ; x)\right) /\left(B_{i}(t ; x)\right)$ for $i=1,2$. Then

$$
\begin{aligned}
& \frac{C_{1}\left(t ; f_{1}(t ; x), f_{2}(t ; x)\right)}{E\left(t ; f_{1}(t ; x), f_{2}(t ; x)\right)}=x_{1} \\
& \frac{C_{2}\left(t ; f_{1}(t ; x), f_{2}(t ; x)\right)}{E\left(t ; f_{1}(t ; x), f_{2}(t ; x)\right)}=x_{2}
\end{aligned}
$$

for a general $\left(x_{1}, x_{2}\right) \in \mathbb{C}^{2}$ (cf. Proposition (1.25)). Now differentiating (4.3.3) with respect to $t$, we get

$$
\frac{d}{d t}\left(\frac{C_{1}\left(t ; f_{1}, f_{2}\right)}{E\left(t ; f_{1}, f_{2}\right)}\right)=0
$$

Therefore

$$
\begin{aligned}
\left(\frac{\partial C_{1}}{\partial t}\right. & \left(t ; f_{1}, f_{2}\right)+\frac{\partial C_{1}}{\partial U_{1}}\left(t ; f_{1}, f_{2}\right) \frac{d f_{1}}{d t}\left(t ; x_{1}, x_{2}\right) \\
& \left.+\frac{\partial C_{1}}{\partial U_{2}}\left(t ; f_{1}, f_{2}\right) \frac{d f_{2}}{d t}\left(t ; x_{1}, x_{2}\right)\right) E\left(t ; f_{1}, f_{2}\right) \\
& -C_{1}\left(t ; f_{1}, f_{2}\right)\left(\frac{\partial E}{\partial t}\left(t ; f_{1}, f_{2}\right)+\frac{\partial E}{\partial U_{1}}\left(t ; f_{1}, f_{2}\right) \frac{d f_{1}}{d t}\left(t ; x_{1}, x_{2}\right)\right. \\
& \left.+\frac{\partial E}{\partial U_{2}}\left(t ; f_{1}, f_{2}\right) \frac{d f_{2}}{d t}\left(t ; x_{1}, x_{2}\right)\right)=0 .
\end{aligned}
$$

Since $t \mapsto\left(t, f_{1}, f_{2}\right)$ is the solution of (3.11), we have

$$
\begin{aligned}
& \frac{d f_{1}}{d t}\left(t ; x_{1}, x_{2}\right)=\frac{F_{1}\left(t ; f_{1}, f_{2}\right)}{G_{1}\left(t ; f_{1}, f_{2}\right)}, \\
& \frac{d f_{2}}{d t}\left(t ; x_{1}, x_{2}\right)=\frac{F_{2}\left(t ; f_{1}, f_{2}\right)}{G_{2}\left(t ; f_{2}, f_{2}\right)} .
\end{aligned}
$$

Substituting (4.3.6) in (4.3.5), we get

$$
\begin{aligned}
& \left(\frac{\partial C_{1}}{\partial t}+\frac{\partial C_{1}}{\partial U_{1}} \frac{F_{1}}{G_{1}}+\frac{\partial C_{1}}{\partial U_{2}} \frac{F_{2}}{G_{2}}\right) E-C_{1}\left(\frac{\partial D_{1}}{\partial t}+\frac{\partial D_{1}}{\partial U_{1}} \frac{F_{1}}{G_{1}}+\frac{\partial D_{2}}{\partial U_{2}} \frac{F_{2}}{G_{2}}\right) \\
& \left(t ; f_{1}, f_{2}\right)=0 .
\end{aligned}
$$

Mutiplying $G_{1} G_{2}$, we get from (4.3.7)

$$
\begin{aligned}
& {\left[\left(G_{1} G_{2} \frac{\partial C_{1}}{\partial t}+G_{2} \frac{\partial C_{1}}{\partial U_{1}} F_{1}+G_{1} \frac{\partial C_{1}}{\partial U_{2}} F_{2}\right) E\right.} \\
& \left.\quad-C_{1}\left(G_{1} G_{2} \frac{\partial D_{1}}{\partial t}+G_{2} \frac{\partial D_{1}}{\partial U_{1}} F_{1}+G_{1} \frac{\partial D_{1}}{\partial U_{2}} F_{2}\right)\right]\left(t ; f_{1}, f_{2}\right)=0
\end{aligned}
$$


Applying Proposition (1.25) for $\mathbb{A}_{\mathrm{C}}^{2}$, we may replace $f_{1}, f_{2}$ by variables: we have a polynomial identity

$$
\begin{aligned}
\left(G_{1} G_{2} \frac{\partial C_{1}}{\partial t}\right. & \left.+G_{2} \frac{\partial C_{1}}{\partial U_{1}} F_{1}+G_{1} \frac{\partial C_{1}}{\partial U_{2}} F_{2}\right) E \\
& -C_{1}\left(G_{1} G_{2} \frac{\partial E}{\partial t}+G_{2} \frac{\partial E}{\partial U_{1}} F_{1}+G_{1} \frac{\partial E}{\partial U_{2}} F_{2}\right)=0
\end{aligned}
$$

in $L\left[U_{1}, U_{2}\right]$. Since the polynomials $C_{1}$ and $E$ are relatively prime in $L\left[U_{1}, U_{2}\right]$ there exists a polynomial $\lambda_{1}\left(t ; U_{1}, U_{2}\right) \in L\left[U_{1}, U_{2}\right]$ such that

$$
G_{1} G_{2} \frac{\partial C_{1}}{\partial t}+G_{2} F_{1} \frac{\partial C_{1}}{\partial U_{1}}+G_{1} F_{2} \frac{\partial C_{1}}{\partial U_{2}}=\lambda_{1} C_{1}
$$

which is an equality in the polynomial ring $L\left[U_{1}, U_{2}\right]$. Similarly there exists a polynomial $\lambda_{2}\left(t ; U_{1}, U_{2}\right) \in L\left[U_{1}, U_{2}\right]$ such that

$$
G_{1} G_{2} \frac{\partial E}{\partial t}+G_{2} F_{1} \frac{\partial E}{\partial U_{1}}+G_{1} F_{2} \frac{\partial E}{\partial U_{2}}=\lambda_{2} E .
$$

It follows from (4.3.9) that $\left(\lambda_{1}-\lambda_{2}\right) C_{1} E=0$ hence $\lambda_{1}=\lambda_{2}=\lambda$ since $\left(u_{1}, u_{2}\right)$ $\mapsto\left(\frac{C_{1}\left(t ; u_{1}, u_{2}\right)}{E\left(t ; u_{1}, u_{2}\right)}, \frac{C_{2}\left(t ; u_{1}, u_{2}\right)}{E\left(t ; u_{1}, u_{2}\right)}\right)$ is birational. Let us set, to simplify the notation $L_{1}=G_{1} G_{2}, L_{2}=G_{2} F_{1}, L_{3}=G_{1} F_{2}$. Then $L_{1}, L_{2}, L_{3} \in R\left[U_{1}, U_{2}\right]$ or their coefficients are known functions. Therefore $C_{1}, E \in L\left[U_{1}, U_{2}\right]$ are $\mathbb{C}$ linearly independent, relatively prime and absolutely irreducible solutions of linear differential equation

$$
L_{1} \frac{\partial B}{\partial t}+L_{2} \frac{\partial B}{\partial U_{1}}+L_{3} \frac{\partial B}{\partial U_{2}}-\lambda B=0
$$

By the same argument, we can show that $C_{2}$ also satisfies the differential equation (4.3.10). $C_{1}, C_{2}, E \in L\left[U_{1}, U_{2}\right]$ are absolutely irreducible and relatively prime solutions of (4.3.10). Thus the theorem for $X=\mathbb{A}^{2}$ follows from (4.3.10) if we can show that the coefficients of the polynomial $\lambda\left(t ; U_{1}, U_{2}\right) \in R Q$. But this is not ture. We have to normalize $\lambda$. Since $L_{1} \neq 0$, there exists $\left(u_{1}, u_{2}\right) \in \mathbb{C}$ by Proposition (1.25) such that $L_{1}\left(t ; u_{1}, u_{2}\right)$ $\neq 0$. Therefore we may further assume $L_{1}(t, 0,0) \neq 0$. Let $g(t)$ be a holomorphic function on a subdomain of $D$. Let us calculate the differential equation satisfied by $g C_{1}, g C_{2}, g D$.

$$
L_{1} \frac{d}{d t}\left(g C_{1}\left(t ; f_{1}(t ; x), f_{2}(t ; x)\right)\right.
$$




$$
\begin{aligned}
& =L_{1}\left(\frac{\partial g C_{1}}{\partial t}\left(t ; f_{1}, f_{2}\right)+\frac{\partial g C_{1}}{\partial U_{1}} \frac{d f_{1}}{d t}+\frac{\partial g C_{1}}{\partial U_{2}} \frac{d f_{2}}{d t}\right) \\
& =L_{1}\left(\frac{\partial g C_{1}}{\partial t}+\frac{\partial g C_{1}}{\partial U_{1}} \frac{F_{1}}{G_{1}}+\frac{\partial g C_{1}}{\partial U_{2}} \frac{F_{2}}{G_{2}}\right)\left(t ; f_{1}, f_{2}\right) \\
& =\left(L_{1} \frac{\partial g C_{1}}{\partial t}+L_{2} \frac{\partial g C_{1}}{\partial U_{1}}+L_{3} \frac{\partial g C_{2}}{U_{2}}\right)\left(t ; f_{1}, f_{2}\right) .
\end{aligned}
$$

On the other hand

$$
\begin{aligned}
L_{1} \frac{d}{d t} & \left(g C_{1}\left(t ; f_{1}(t ; x), f_{2}(t ; x)\right)\right) \\
= & L_{1} \frac{d g}{d t} C_{1}\left(t ; f_{1}, f_{2}\right)+L_{1} g \frac{d}{d t} C_{1}\left(t ; f_{1}, f_{2}\right) \\
= & L_{1} \frac{d g}{d t} C_{1}\left(t ; f_{1}, f_{2}\right)+L_{1} g\left(\frac{\partial C_{1}}{\partial t}\left(t ; f_{1}, f_{2}\right)+\frac{\partial C_{1}}{\partial U_{1}} \frac{d f_{1}}{d t}+\frac{\partial C_{1}}{\partial U_{2}} \frac{d f_{2}}{d t}\right) \\
= & L_{1} \frac{d g}{d t} C_{1}\left(t ; f_{1}, f_{2}\right)+L_{1} g\left(\frac{\partial C_{1}}{\partial t}\left(t ; f_{1}, f_{2}\right)+\frac{\partial C_{1}}{\partial U_{1}} \frac{F_{1}}{G_{1}}\left(t ; f_{1}, f_{2}\right)\right. \\
& \left.+\frac{\partial C_{1}}{\partial U_{2}} \frac{F_{2}}{G_{2}}\left(t ; f_{1}, f_{2}\right)\right) \\
= & L_{1} \frac{d g}{d t} C_{1}\left(t ; f_{1}, f_{2}\right)+g\left(L_{1} \frac{\partial C_{1}}{\partial t}+L_{2} \frac{\partial C_{1}}{\partial U_{2}}+L_{3} \frac{\partial C_{1}}{\partial U_{2}}\right)\left(t ; f_{1}, f_{2}\right) \\
= & \left(L_{1} \frac{d g}{d t} C_{1}+g \lambda C_{1}\right)\left(t ; f_{1}, f_{2}\right) .
\end{aligned}
$$

Therefore we have

$$
\begin{aligned}
& L_{1} \frac{\partial g C_{1}}{\partial t}\left(t ; U_{1}, U_{2}\right)+L_{2} \frac{\partial g C_{1}}{\partial U_{1}}\left(t ; U_{1}, U_{2}\right)+L_{3} \frac{\partial g C_{2}}{\partial U_{2}}\left(t ; U_{1}, U_{2}\right) \\
& \quad=\left(L_{1} g^{-1} g^{\prime}+\lambda\right) g C_{1}\left(t ; U_{1}, U_{2}\right) .
\end{aligned}
$$

The polynomials $g C_{2}$ and $g E$ satisfy the same equation (4.3.11) too. Thus we can choose $g$ so that $L_{1}(t, 0,0) g^{-1}(d g / d t)-\lambda(t, 0,0)=0$. Therefore we may assume that for $\lambda$ in (4.3.10), we have $\lambda(t ; 0,0)=0$. Under this hypothesis we show that every coefficient of the polynomial $\lambda\left(t ; U_{1}, U_{2}\right)$ is algebraic over the differential field $F$ generated by the coefficients of $L_{1}, L_{2}, L_{3}$ over $\mathbb{C}$ hence in $R Q$. Assume now that some of the coefficients of the polynomial $\lambda\left(t ; U_{1}, U_{2}\right)$ is not algebraic over $F$. We apply Lemma (4.2): we take for the set $\left\{a_{1}, a_{2}, \cdots, a_{l}\right\}$ the set coefficients of the polynomials $L_{1}, L_{2}, L_{3}$, for the set $\left\{b_{1}, b_{2}, \cdots, b_{m}\right\}$ the set of coefficients of the polynomial $\lambda\left(t ; U_{1}, U_{2}\right)$ and for the set $\left\{c_{1}, c_{2}, \cdots, c_{n}\right\}$ the set of coefficients 
of the polynomials $C_{1}\left(t ; U_{1}, U_{2}\right), E\left(t ; U_{1}, U_{2}\right)$. The lemma shows that we can ceform $\lambda$ and the solutions $C_{1}$ and $E$ of the differential equation (4.3.10). Namely we can find a subdomain $D^{\prime}$ of $D$, a domain $D_{1} \subset \mathbb{C}$ and a point $s_{0} \in D_{1}$ such that the differential equation (4.3.10) and the solutions $C_{1}\left(t ; U_{1}, U_{2}\right), E\left(t ; U_{1}, U_{2}\right)$ are parametrized by $D_{1}$ :

$$
L_{1} \frac{\partial B}{\partial t}+L_{2} \frac{\partial B}{\partial U_{1}}+L_{3} \frac{\partial B}{\partial U_{2}}-\tilde{\lambda}(s) B=0
$$

where $\tilde{\lambda}(s)=\tilde{\lambda}\left(t, s ; U_{1}, U_{2}\right)$ is a polynomial with holomorphic coefficients over $D^{\prime} \times D^{1}$ and $\lambda\left(t, s_{0} ; U_{1}, U_{2}\right)=\lambda\left(t ; U_{1}, U_{2}\right), \tilde{\lambda}(t, s ; 0,0)=0$ since we deform only non-zero coefficients. The differential equation (4.3.11)' has solutions $\tilde{C}_{1}\left(t, s ; U_{1}, U_{2}\right)$ and $\tilde{E}\left(t, s ; U_{1}, U_{2}\right)$ which are polynomial in $U_{1}, U_{2}$ with coefficients holomorphic over $D^{\prime} \times D_{1}$ and $\tilde{C}_{1}\left(t, s_{0} ; U_{1}, U_{2}\right)=C_{1}\left(t ; U_{1}, U_{2}\right)$, $\tilde{E}\left(t, s_{0} ; U_{1}, U_{2}\right)=E\left(t ; U_{1}, U_{2}\right)$. Moreover we may assume that $\tilde{\lambda}(s)$ is effectively parametrized (i.e. if $s_{1}, s_{2} \in D_{1}$ be different points, then $\tilde{\lambda}\left(s_{1}\right) \neq \tilde{\lambda}\left(s_{2}\right)$ ). Let $S_{0}$ be the ring of holomorphic functions on $D^{\prime}$ generated over $\mathbb{C}$ by the coefficients of the polynomials $C_{1}\left(t ; U_{1}, U_{2}\right), E\left(t ; U_{1}, U_{2}\right)$ and $S$ be the ring of holomorphic functions on $D^{\prime} \times D$ generated over $\mathbb{C}$ by the coefficients of the polynomials $\tilde{C}_{1}\left(t, s ; U_{1}, U_{2}\right), \tilde{E}\left(t, s ; U_{1}, U_{2}\right)$. It follows from Lemma (4.2) that the morphism $S \rightarrow S_{0}$ of substitution $s=s_{0}$ is an isomorphism. Therefore the polynomials $\tilde{C}_{1}\left(t, s ; U_{1}, U_{2}\right)$ and $\tilde{E}\left(t, s ; U_{1}, U_{2}\right)$ are absolutely irreducible over the quotient field of $S^{\prime}$ and relatively prime. We have $(\partial / \partial t)\left(\tilde{C}_{1} / \tilde{E}\right)\left(t, s ; f_{1}(t ; x), f_{2}(t, x)\right)=0$ since $\left(\partial \tilde{C}_{1} / \partial t\right)\left(t, s ; f_{1}, f_{2}\right)=\tilde{\lambda} \tilde{C}_{1}\left(t ; f_{1}, f_{2}\right)$, $(\partial \tilde{E} / \partial t)\left(t, s ; f_{1}, f_{2}\right)=\tilde{\lambda} \tilde{E}\left(t, s ; f_{1}, f_{2}\right)$ by the differential equation (3.11). Namely if we fix $s \in D_{1}$, then $\left(\tilde{C}_{1} / \tilde{E}\right)\left(t, s ; U_{1}, U_{2}\right)$ is a first integral of the system (3.11) of Pfaffian differential equations. Therefore there exists a holomorphic function $\varphi_{s}$ of 2 -variables such that $\left(\tilde{C}_{1} / \tilde{E}\right)\left(t, s ; U_{1}, U_{2}\right)=\varphi_{s}\left(\left(C_{1} / E\right)\left(t ; U_{1}, U_{2}\right)\right.$, $\left.\left(C_{2} / E\right)\left(t ; U_{1}, U_{2}\right)\right)$ in a neighbourhood $W$ of $\left(t_{0}, s_{0}\right)$. If we substitute $U_{1}=$ $\left(A_{1} / B_{1}\right)(t ; x), U_{2}=\left(A_{2} / B_{2}\right)(t ; x)$ for a general $\left(x_{1}, x_{2}\right) \in \mathbb{A}_{\mathrm{C}}^{2}=\mathbb{C}^{2}$ (cf. Proposition (1.25)), we get $\left(\tilde{C}_{1} / \tilde{E}\right)\left(t, s ; A_{1} / B_{1}, A_{2} / B_{2}\right)=\varphi_{s}\left(\left(C_{1} / E\right)\left(t ; A_{1} / B_{1}, A_{2} / B_{2}\right),\left(C_{2} E\right)(t\right.$; $\left.\left.A_{1} / B_{1}, A_{2} / B_{2}\right)\right)=\varphi_{s}\left(x_{1}, x_{2}\right)$. If we put $t=t_{0}$, we get $\left(\tilde{C}_{1} / \tilde{E}\right)\left(t_{0}, s ; x_{1}, x_{2}\right)=\varphi_{s}\left(x_{1}, x_{2}\right)$. Therefore putting $I\left(s ; U_{1}, U_{2}\right)=\tilde{C}_{1}\left(t_{0}, s ; U_{1}, U_{2}\right)$ and $J\left(s ; U_{1}, U_{2}\right)=\tilde{E}\left(t_{0}, s\right.$; $\left.U_{1}, U_{2}\right)$, we have

$$
\frac{\tilde{C}_{1}}{\tilde{E}}\left(t, s ; U_{1}, U_{2}\right)=\frac{I}{J}\left(s ; \frac{C_{1}}{E}\left(t ; U_{1}, U_{2}\right), \frac{C_{2}}{E}\left(t ; U_{1}, U_{2}\right)\right)
$$

and 


$$
\frac{\tilde{C}_{1}}{\tilde{E}}\left(t, s ; \frac{A_{1}\left(t ; X_{1}, X_{2}\right)}{B_{1}\left(t ; X_{1}, X_{2}\right)}, \frac{A_{2}\left(t ; X_{1}, X_{2}\right)}{B_{2}\left(t ; X_{1}, X_{2}\right)}\right)=\frac{I}{J}\left(s ; X_{1}, X_{2}\right) .
$$

Recall that $\left(U_{1}, U_{2}\right) \mapsto\left(C_{1} / E, C_{2} / E\right)$ is the birational automorphism of $\mathbb{A}_{L}^{2}$. Let $N$ be the field of meromorphic functions on $D \times D_{1}$ generated over $L$ by the coefficients of polynomials $I\left(s, t ; U_{1}, U_{2}\right), J\left(s, t ; U_{1}, U_{2}\right) . \quad L$ and $N$ are subfields of the field $\Omega$ of all the meromorphic functions on $D^{\prime} \times D_{1}$. We denote by $\bar{\Omega}$ an algebraic closure of $\Omega$. We denote by $\Phi$ the birational automorphism of $\mathbb{P}_{\bar{\Omega}}^{2}$ defined by $\left(x_{1}, x_{2}\right) \mapsto\left(\left(A_{1} / B_{1}\right)\left(x_{1}, x_{2}\right),\left(A_{1} / B_{2}\right)\left(x_{1}, x_{2}\right)\right)$. Let $Z \subset \mathbb{P}_{\bar{\Omega}}^{2} \times \mathbb{P}_{\bar{\Omega}}^{2}$ be the graph of the birational correspondence $\Phi$. For a curve $\gamma$ on $\mathbb{P}_{\bar{\delta}}^{2}$, we denote $p_{2}\left(p_{1}^{-1} \gamma \cap Z\right)$ (resp. $p_{1}\left(p_{2}^{-1} \gamma \cap Z\right)$ ) by $\Phi^{* \gamma}$ (resp. $\Phi_{*}^{-1} \gamma$ ) where $p_{i}: \mathbb{P}^{2} \times \mathbb{P}^{2} \rightarrow \mathbb{P}^{2}$ is the projection $(i=1,2)$; namely $\Phi_{*}\left(\right.$ resp. $\left.\Phi_{*}^{-1}\right)$ is the morphism of the group of 1-cycles to itself defined by the correspondence $\Phi$ (resp. $\left.\Phi^{-1}\right)$. It follows from (4.3.12a) that the zero locus $V\left(\tilde{C}_{1}\right)$ of $\tilde{C}_{1}$ is an irreducible component of $\Phi_{*}^{-1}(V(I)) \cup \Phi_{*}^{-1}$ (polar divisors of $J$ ) $=$ the proper transform of $V(I) \cup$ ssome curves $\left.\gamma \subseteq A_{\Omega}^{2} \mid \Phi_{*}(\gamma)=0\right\} \cup \Phi_{*}^{-1}$ (polar divisors of $J$ ). Since the polar divisor of $J$ is the line at infinity, the curve $\Phi_{*}^{-1}$ (the polar divisor of $J$ ) is defined over $L$. Since $\Phi$ is defined over $L$, we get

(4.3.13) $V\left(\tilde{C}_{1}\right)$ is an irreducible component of (the proper transform of $V(I)) \cup($ cruves defined on $L$ ). Now we study two cases: (4.3.14) the curve $V\left(\tilde{C}_{1}\right)$ is defined over $L ;(4.3 .15)$ the curve $V\left(\tilde{C}_{1}\right)$ is an irreducible component of the proper transform of $V(I)$. We show that we are always in case (4.3.15). In the first case (4.3.14), there exists a polynomial $C^{\prime}\left(U_{1}, U_{2}\right) \in$ $L\left[U_{1}, U_{1}\right]$ such that $\tilde{C}_{1}=\alpha C^{\prime}\left(U_{1}, U_{2}\right)$ with $\alpha \in \bar{\Omega}$. Comparing the coefficients, $\alpha=\alpha(s, t) \in N$. Since the coefficients of $\tilde{C}_{1}$ is holomorphic at $s=s_{0}$ and $\tilde{C}_{1}\left(t, s_{0} ; U_{1}, U_{2}\right)=C_{1}\left(t ; U_{1}, U_{2}\right), \alpha$ is holomorphic at $s=s_{0}$. We may therefore assume that $C^{\prime}=C_{1}\left(t ; U_{1}, U_{2}\right)$ and $\tilde{C}_{1}\left(s, t ; U_{1}, U_{2}\right)=\alpha(s, t) C_{1}\left(t ; U_{1}, U_{2}\right)$. It follows from (4.3.10) and (4.3.11) that

$$
\begin{aligned}
0 & =L_{1} \frac{\partial \tilde{C}_{1}}{\partial t}+L_{2} \frac{\partial \tilde{C}_{1}}{\partial U_{1}}+L_{3} \frac{\partial \tilde{C}_{1}}{\partial U_{3}}-\tilde{\lambda} \tilde{C}_{1} \\
& =L_{1}\left(\frac{\partial \alpha}{\partial t} C_{1}+\alpha \frac{\partial C_{1}}{\partial t}\right)+\alpha L_{2} \frac{\partial C_{1}}{\partial U_{1}}+\alpha L_{3} \frac{\partial C_{1}}{\partial U_{2}}-\tilde{\lambda} \alpha C_{1} \\
& =L_{1} \frac{\partial \alpha}{\partial t} C_{1}+\alpha\left(L_{1} \frac{\partial C_{1}}{\partial t}+L_{2} \frac{\partial C_{1}}{\partial U_{1}}+L_{3} \frac{\partial C_{1}}{\partial U_{3}}\right)-\tilde{\lambda} \alpha C_{1} \\
& =L_{1} \frac{\partial \alpha}{\partial t} C_{1}+\alpha \lambda C_{1}-\tilde{\lambda} \alpha C_{1} .
\end{aligned}
$$

Hence $L_{1} \frac{\partial \alpha}{\partial t}+\alpha \lambda-\tilde{\lambda} \alpha=0$. Consequently, $\frac{\partial \alpha}{\partial t}=0$ since the constant 
terms of the polynomials $\lambda, \tilde{\lambda}$ are 0 and that of $L_{1}$ is not zero. Therefore $\alpha(s, t)=\alpha(s)$ is a function of $s$. Then

$$
\begin{aligned}
0 & =L_{1} \frac{\partial \alpha C_{1}}{\partial t}+L_{2} \frac{\partial \alpha C_{1}}{\partial U_{1}}+L_{3} \frac{\partial \alpha C_{1}}{\partial U_{2}}-\tilde{\lambda} \alpha C_{1} \\
& =\alpha\left(L_{1} \frac{\partial C_{1}}{\partial t}+L_{2} \frac{\partial C_{1}}{\partial U_{1}}+L_{3} \frac{\partial C_{1}}{\partial U_{2}}-\tilde{\lambda} C_{1}\right) \\
& =\alpha\left(\lambda C_{1}-\tilde{\lambda} C_{1}\right) \\
& =\alpha C_{1}(\lambda-\tilde{\lambda})
\end{aligned}
$$

by (4.3.10) and (4.3.11). Hence $\lambda=\tilde{\lambda}$ this contradicts the choice of $\bar{\lambda}$. Hence we assume that we are in case (4.3.15). We choose an integer $n$ such that $\tilde{I}\left(s ; Y_{0}, Y_{1}, Y_{2}\right)=Y_{0}^{n}, I\left(s ; Y_{1} / Y_{0}, Y_{2} / Y_{0}\right)$ is a polynomial. By the same argument using (4.3.12b) as in (4.3.13), $V(\tilde{I})$ is contained in (the proper transform of $\left.V\left(\tilde{C}_{1}\right)\right) \cup($ curves defined over $L$ and hence at most one component $V\left(\tilde{I}\left(s, E\left(t ; U_{1}, U_{2}\right), C_{1}\left(t ; U_{1}, U_{2}\right), C_{2}\left(t ; U_{1}, U_{2}\right)\right)\right.$ is not defined over $L$. It follows from the hypotheses (4.3.15) and (4.3.13) that there exist $\mu \in \bar{Q}$ and a polynomial $Q \neq 0$ in $L\left[U_{1}, U_{2}\right]$ such that

$$
\begin{gathered}
\tilde{I}\left(s, E\left(U_{1}, U_{2}\right), C_{1}\left(t: U_{1}, U_{2}\right), C_{2}\left(t ; U_{1}, U_{2}\right)\right) \\
=\mu(t, s) Q\left(t ; U_{1}, U_{2}\right) \tilde{C}_{1}\left(t, s ; U_{1}, U_{2}\right) .
\end{gathered}
$$

Comparing the constant terms, we know $\mu(t, s) \in N$. We substitute $U_{1}=$ $f_{1}\left(t ; x_{1}, x_{2}\right), U_{2}=f_{2}\left(t ; x_{1}, x_{2}\right)$ in (4.3.13) and take $d / d t$ log.

The left hand side is equal to

$$
\begin{aligned}
& \tilde{I}^{-1}\left(\frac{\partial \tilde{I}}{\partial E} \frac{d E}{d t}+\frac{\partial \tilde{I}}{\partial C_{1}} \frac{d C_{1}}{d t}+\frac{\partial \tilde{I}}{\partial C_{2}} \frac{d C_{2}}{d t}\right)\left(t ; f_{1}, f_{2}\right) \\
& \quad=\tilde{I}^{-1} L_{1}^{-1}\left(\frac{\partial \tilde{I}}{\partial E} \lambda E+\frac{\partial \tilde{I}}{\partial C_{1}} \lambda C_{1}+\frac{\partial \tilde{I}}{\partial C_{2}} \lambda C_{2}\right)\left(t ; f_{1}, f_{2}\right) \\
& \quad=\text { (degree of the homogeneous polynomial } \tilde{I}) \lambda L_{1}^{-1}\left(t ; f_{1}, f_{2}\right) .
\end{aligned}
$$

The right handside is equal to

$$
\begin{gathered}
\left(\mu^{-1} \frac{\partial \mu}{\partial t}\right)(t, s)+Q^{-1}\left(\frac{\partial Q}{\partial t}\right)\left(t ; f_{1}, f_{2}\right)+\tilde{C}_{1}^{-1} \frac{\partial \tilde{C}_{1}}{\partial t}\left(t ; f_{1}, f_{2}\right) \\
=\mu^{-1} \frac{\partial \mu}{\partial t}(t, s)+Q^{-1}\left(\frac{\partial Q}{\partial t}\right)\left(t ; f_{1}, f_{2}\right)+\tilde{\lambda}\left(t, s ; f_{1}, f_{2}\right) L_{1}^{-1}
\end{gathered}
$$

We get thus

$$
\begin{aligned}
(\operatorname{deg} \tilde{I}) \cdot \lambda L_{1}^{-1}\left(t ; f_{1}, f_{2}\right)= & \mu^{-1} \frac{\partial \mu}{\partial t}(t, s)+Q^{-1}\left(\frac{\partial Q}{\partial t}\right)\left(t ; f_{1}, f_{2}\right) \\
& +\tilde{\lambda} L_{1}^{-1}\left(t ; f_{1}, f_{2}\right) .
\end{aligned}
$$


Thus we get

$$
\begin{aligned}
\tilde{\lambda}\left(t, s ; f_{1}, f_{2}\right)= & (\operatorname{deg} \tilde{I}) \lambda\left(t ; f_{1}, f_{2}\right)-L_{1} Q^{-1} \frac{\partial Q}{\partial t}\left(t ; f_{1}, f_{2}\right) \\
& -L_{1}\left(t ; f_{1}, f_{2}\right) \mu^{-1} \frac{\partial \mu}{\partial t}(t, s) .
\end{aligned}
$$

In particular $L_{1} Q^{-1} \frac{\partial Q}{\partial t}\left(t ; f_{1}, f_{2}\right)=\Psi\left(t ; f_{1}, f_{2}\right)$ is a polynomial in $f_{1}$ and $f_{2}$. Therefore by Proposition (1.25), we get a polynomial identity in $U_{1}$ and $U_{2}$

$$
\begin{gathered}
\tilde{\lambda}\left(t, s ; U_{1}, U_{2}\right)=(\operatorname{deg} \tilde{I}) \lambda\left(t ; U_{1}, U_{2}\right)-\Psi\left(t ; U_{1}, U_{2}\right) \\
-L_{1}\left(t ; U_{1}, U_{2}\right) \mu^{-1} \frac{\partial \mu}{\partial t}(t, s) .
\end{gathered}
$$

We compare the constant terms in (4.3.19) and get

$$
0=-\Psi(t ; 0,0)-L_{1}(t ; 0,0) \mu^{-1} \frac{\partial \mu}{\partial t}(t, s)
$$

since $\tilde{\lambda}(t, s ; 0,0)=\lambda(t ; 0,0)=0$. As $L_{1}(t ; 0,0) \neq 0, \quad$ (4.3.20) shows that $\mu^{-1} \frac{\partial \mu}{\partial t}(t, s)$ does not depend on $s$. Hence in the equality (4.3.19), the right hand side is independent of $s$ and consequently $\tilde{\lambda}\left(t, s ; U_{1}, U_{2}\right)$ is independent of $s$. This contradicts the choice of $\tilde{\lambda}$ which is effectively parametrized by $s \in D_{1}$.

Now we study the case $q(X)=0$. We take a non-singular projective model $\bar{X}$ of $X$. Since $q=0$, the Picard functor Pic $(X / \mathbb{C})$ on the category of $\mathbb{C}$-schemes is representable by a finitely generated commutative discrete group $G$. Let $g_{1}, g_{2}, \cdots, g_{l}$ be a system of generators of $G$. There exists a non-empty affine open set of $\bar{X}$ on which the restrictions of the generators $g_{1}, g_{2}, \cdots, g_{2}$ are the trivial line bundle. We take as $X$ this affine open set. Therefore the Picard group $\operatorname{Pic} X / \mathbb{C}(\mathbb{C})=0$, namely the coordinate ring $\mathbb{C}[X]$ of the affine variety $X$ is U.F.D. Let $K \supset \mathbb{C}$ be a field extension. Then Pic $\bar{X} \otimes_{\mathrm{C}} K / K$ is representable by $K$-algebraic group $G$. Hence Pic $\bar{X}$ $\otimes_{\mathrm{c}} K / K(K)=G$ and $G$ is generated by the divisors defined over $\mathbb{C}$ and whose supports are in $(\bar{X}-X) \otimes_{\mathrm{C}} K$. We have thus Pic $X \otimes_{\mathrm{c}} k(K)=0$, or the coordinate ring $K\left[X \otimes_{C} K\right]$ of the algebraic variety $X \otimes_{C} K$ defined over $K$ is U.F.D. We have shown that (i) $X \otimes_{\mathrm{c}} K / K$ is smooth and (ii) its coordinate ring is U.F.D. In the proof of the Theorem for $\mathbb{A}^{2}$, we used only these two particular properties (i), (ii) of $\mathbb{A}^{2}$. Therefore the proof for $\mathbb{A}^{2}$ works without any modification also for $X$.

Now it remains to treat the general case. We have so far proved 
the theorem for two cases (i) $\operatorname{dim} X=1$ and (ii) $q(X)=0$. We show below that

(4.4) if $q(X)>0$, then the solution of the system (3.11) of Pfaffian differential equations is reduced to by the permissible operations that of a system of Pfaffian differential equations

(4.5) $d w_{i}=\frac{J_{j}\left(t ; w_{1}, w_{2}, \cdots, w_{l}\right)}{K_{i}\left(t ; w_{1}, w_{2}, \cdots, w_{l}\right)} d t 1 \leqq i \leqq l$ on an algebraic variety $Y$ such that $\operatorname{dim} Y<\operatorname{dim} X$, the $J_{i}$ and the $K_{i}$ are polynomials with known functions by the permissible operations in $\S 2$ from $R$ and such that solution of (4.5) depends rationally on the initial conditions. This combined with the special cases (i) and (ii) proves the Theorem by induction on the dimension of $X$.

Let us therefore prove (4.4). The proof is similar to the latter part of the proof of Theorem (2.19). In fact, if we choose a non-singular projective model $\bar{X}$ and we take as $X$ a non-empty Zariski open set of $X$, then we have a morphism $f: \bar{X} \rightarrow \operatorname{Alb} \bar{X}$. Since Alb $\bar{X}$ is birational invariant, we have a morphism of group functors $\varphi: \operatorname{Bir} \bar{X} \rightarrow \operatorname{Aut}(\operatorname{Alb} X)$ on the category of $\mathbb{C}$-schemes.

Let $L$ be a field extension of $K$ such that we have an $L$-rational point $F: D \rightarrow \operatorname{Bir} \bar{X}$ of $\operatorname{Bir} \bar{X}$ defining a general solution of the system (3.11) of Pfaffian differential equations (cf. $\S 3$ ). Note that the Lie algebra of the group functor $\operatorname{Bir} \bar{X}$ on the category of $\mathbb{C}$-schemes is the $\mathbb{C}$-vector space $\operatorname{Der}_{\mathbf{c}}(\mathbb{C}(X), \mathbb{C}(X))$ of the $\mathbb{C}$-derivations. We consider the composite $\varphi \circ F: D$ $\rightarrow \operatorname{Aut}(\operatorname{Alb} X)$. Since we may assume that there exists a point $t_{0} \in D$ such that $F\left(t_{0}\right)=\operatorname{Id}_{X}, \varphi \circ F(D) \subset \operatorname{Aut}^{\circ}(\operatorname{Alb} X)=\operatorname{Alb} X$, where Aut $^{0}$ denote the component of 1 and Alb $X$ acts on Alb $X$ through the addition. $\varphi \circ F: D$ $\rightarrow$ Aut $^{0}(\operatorname{Alb} X)$ defines a vector field $\Theta(\varphi \circ F)$ on $(\operatorname{Alb} X) \otimes_{\mathrm{c}} L$ as in $\S 1$ or a system $\operatorname{Pf}(\varphi \circ F)$ of Pfaffian differential equations of rank $\operatorname{dim} \operatorname{Alb} X$ on $D \times$ Alb $X$ with coefficients in $L$. It follows from the Proof of Proposition (2.9) that $\Theta(\varphi \circ F)=\varphi_{*}(\Theta(F))$ and the vector field $\Theta(\varphi \circ F)$ is defined over the quotient field $K$ of $R$. Hence the coefficients of the system $\operatorname{Pf}(\varphi \circ F)$ of Pfaffian differential equations are in $K$. Therefore $\varphi \circ F: D \rightarrow \operatorname{Alb} X$ is a $K_{1}$-rational point where $K_{1}=K(\{f \circ \varphi \circ F \mid f$ is a rational function on the abelian variety $\mathrm{Alb} \bar{X}\}$ ) and by the operation (Q) $K_{1}$ consists of known functions.

Therefore we get a $K_{1}$-rational point $\psi_{1}: \operatorname{Spec} K_{1} \rightarrow$ Alb $X$ and this point is the image of an $L$-rational point $\psi_{2}: \operatorname{Spec} L \rightarrow \operatorname{Bir} X: \psi_{1}\left(\operatorname{Spec} K_{1}\right)$ $=\varphi \circ \psi_{2}(\operatorname{Spec} L)$, where $L$ is an extension of $\mathbb{C}$ of finite type. Since $\psi_{2}$ is 
defined over a ring $S$ of finite type over $\mathbb{C}$, we get a morphism $\psi_{s}$ : Spec $S$ $\rightarrow \operatorname{Bir} X$ such that $\varphi \circ \psi_{3}$ (the generic point of $\left.\operatorname{Spec} S\right)=\psi_{1}\left(\operatorname{Spec} K_{1}\right)$.

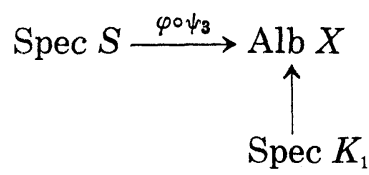

Therefore there exist a finite algebraic extension $K_{2}$ of $K_{1}$ and a $K_{2}$-rational point $\chi:$ Spec $K_{2} \rightarrow S$ such that we have a commutative diagram

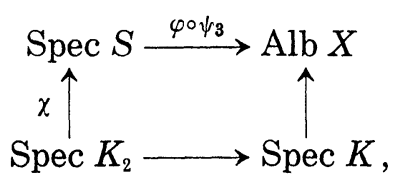

where the second horizontal arrow is the morphism induced by the inclusion $K_{1} \subset K_{2}$. We get finally $K_{2}$-rational point $\operatorname{Spec} K_{2} \stackrel{\varphi_{3^{\circ} x}}{\longrightarrow} \operatorname{Bir} X$ such that $\varphi \circ \varphi_{3} \circ \chi\left(\operatorname{Spec} K_{2}\right)=\psi_{1}\left(\operatorname{Spec} K_{1}\right)$. It follows from Proposition (1.7) that we get a $K_{2}$-rational point $\tilde{F}: D \rightarrow \operatorname{Bim} X$ (here we replace $D$ by an appropriate subdomain if necessary) such that $\varphi \circ \tilde{F}=\varphi \circ F$. By the operation (P2) the field $K_{2}$ also consists of known functions. Now for any $t \in D$, $F \tilde{F}^{-1}(t)=F(t) \tilde{F}(t)^{-1} \in \operatorname{Bir} \bar{X}$ respects the fibration $\varphi: \bar{X} \rightarrow$ Alb $\bar{X}$. Therefore the system of Pfaffiian differential equations associated with $F \tilde{F}^{-1}: D \rightarrow$ Bir $\bar{X}$ are defined over each fibre of $\varphi$. Therefore a general solution of the system $\operatorname{Pf}\left(F \tilde{F}^{-1}\right)$ of Pfaffian differential equations associated with $F \tilde{F}^{-1}$ is a general solution of the system of Pfaffian differential equations over a fibre $Y$ of $\varphi$. Therefore $\operatorname{dim} Y<\operatorname{dim} X$. Since the question is local, we may take an irreducible component of $Y$. Now we show that the system $\operatorname{Pf}\left(\Theta\left(\tilde{F}^{-1} F\right)\right)$ of Pfaffian differential equations is defined over $K_{2}$ which consists of known functions. In fact let $f$ be a rational function on $X$ and let us put $\tilde{F}^{-1}=W$.

$\Theta(W F) f(x)$ is equal to, by definition, $\lim _{h \rightarrow 0} \frac{f\left(W F(t+h)(W F)^{-1}(t) x\right)-f(x)}{h}$.

The latter is equal to

$$
\begin{aligned}
\lim _{h \rightarrow 0} & \frac{f\left(W(t+h) F(t+h) F(t)^{-1} W(t)^{-1} x\right)-f\left(W(t+h) W(t)^{-1} x\right)}{h} \\
& +\frac{f\left(W(t+h) W(t)^{-1} x\right)-f(x)}{h} \\
= & \lim _{h \rightarrow 0} \frac{f\left(W(t) F(t+h) F(t)^{-1} W(t)^{-1} x\right)-f(x)}{h}+\Theta(W)(f)
\end{aligned}
$$


It follows from the Proof of Lemma (2.12) that the first term

$$
\lim _{h \rightarrow 0} \frac{f\left(W(t) F(t+h) F(t)^{-1} W(t)^{-1} x\right)-f(x)}{h}=\left(\operatorname{ad} W(t)^{-1}\right)(\Theta(F))(f)
$$

is in $K_{2}$. Therefore $\Theta(W F) f(x)$ is in $K_{2}$. It follows from induction that there exists an extension $K_{3}$ consisting of known functions such that $W F: D \rightarrow \operatorname{Bir} V$ is $K_{3}$-rational. Since $F=\tilde{F}\left(\tilde{F}^{-1} F\right)=\tilde{F}(W F)$, the general solution that we have chosen is $\tilde{F}(W F)$-orbit or $K_{3}$-rational point. Namely we can express it by using the permissible operations.

COROLlaRY (4.6). Let $R$ be a ring of holomorphic functions on a domain $D$ and $F\left(t ; Y_{0}, Y_{1}, \cdots, Y_{n}\right) \in R\left[Y_{0}, Y_{1}, \cdots, Y_{n}\right]$. If the general solution of an algebraic differential equation $F\left(t ; y(t), y^{\prime}(t), \cdots, y^{(n)}(t)\right)=0$ depends rationally on the initial condition, then the general solution is obtained by a finite iteration of the permissible operations (O), (P1), (P2), . , (P5).

Proof. This is a consequence of Theorem (2.19), Proposition (3.16) and Theorem (4.3).

Concluding remark (4.7). In $\S 1$ we introduced the operation $Q$. Let $F: D \rightarrow G^{\text {an }}$ be an analytic map of a domain $D$ to an algebraic group $G$. Then $F$ defines $\partial F: D \rightarrow$ Lie $G$. The operation $Q$ is to recover $F$ from the derivative $\partial F$.

More generally let $F: D \rightarrow \operatorname{Bim} X$ be a morphism of functors of a domain $D \subset \mathbb{C}$. We can consider as if $\operatorname{Bim} X=(\operatorname{Bir} X)^{\text {an }}$ (cf. Part I). We can associate to $F$ a rational vector field $\Theta(F)$ on $X \otimes_{\mathrm{c}} K$ as in $\S 1$. We can consider $\Theta(F)$ as a map $\Theta(F): D \rightarrow \operatorname{Der}_{\mathrm{c}}(\mathbb{C}(X), \mathbb{C}(X))=\operatorname{Lie}(\operatorname{Bir} X)$ (cf. Proposition (2.6)). Theorem (4.3) says that when $\Theta(F)$ is given, we can recover $F$ by using the operation $Q$. We notice here that in general $\operatorname{Bir} X$ is of infinite dimensional (see [U2]). In this formulation the irreducibility theorem should take the following form: there exist a Lie pseudo-group $G$ of infinite dimension and a holomorphic map $F: D \rightarrow G$ such that we can not reconstruct $F$ from $\partial F: D \rightarrow$ Lie $G$ by using only the operation $Q$. This will be discussed in our future work.

\section{REFERENCES}

[B] Borel, A., Linear algebraic groups, Benjamin, New York, 1969.

[D] Demazure, M., Sous-groupes algébriques de rang maximum du groupe de Cremona, Ann. Sci. Ecole Norm. Sup., 4e série, t.3 (1970), 507-588. 
[Di] Dieudonné, J., Calcul infinitesimal, Collection Methodes, Hermann, Paris, 1968.

[E.G.A.] Grothendieck, A., Élements de géométric algébriuqe, Publ. Math. I.H.E.S., nos $4,7,11, \ldots$, P.U.F., 1960, 1961, ...

[G1] - Technique de construction en géométric analytique VII, Rapport sur les théorèmes de finitude de Grauert et Remmert Sém. H. Cartan 13e année $1960 / 61, \mathrm{n}^{\circ} 15$.

[G2] - Technique de descente et théorème d'existence en géométric algébrique IV, les schemas de Hilbert, Sém. Bourbaki, t. 13, 1960/61, n²21.

[K] Kraus, G., Meromorphe Funktionen auf allgemeinen komplexen Raumen, Math. Ann., 209 (1974), 257-265.

[M] Mumford, D., Algebraic geometry I, Complex projective varieties, Grund. der math. Wiss. 221, Springer, Berlin Heidelberg New York, 1976.

[P] Painlevé, P., Leçons de Stockholm, Euvres de P. Painlevé I, 199-818, Editions du C.N.R.S., Paris, 1972.

[U1] Umemura, H., Sur les sous-groupes algébriques primitifs de groupe de Cremona à trois variables, Nagoya Math. J., 79 (1980), 47-67.

[U2] _- Algebro-geometric problems arising from Painleve's works, Algebraic and Topological Theories-to the memory of Dr. Takehiko MIYATA, 467-495, Kinokuniya, Tokyo, 1985.

[W] Weil, A., On algebraic groups of transformations, Amer. J. Math., 77 (1955), 355-391.

[Z] Zariski, O., Introduction to the problem of minimal models in the theory of algebraic surfaces, Publ. of Math. Soc. of Japan, Tokyo, 1958.

Footnote (1). As it is not clear that $\mathscr{Z}$ is birational at $\eta=(h(p), x)$ for a general point $x \in X$, we give here a complete proof. The following argument was suggested by Hiroshi Saito. We denote by $q^{\prime}: Z_{U} \rightarrow U \times X$ the restriction to $Z_{U^{\prime}}$ of the projection $p_{12}: U \times X \times X \rightarrow U \times X$. The diagram

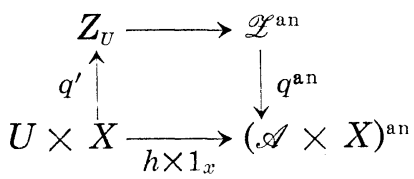

is cartesian in the category of the analytic spaces. In particular there exists the unique point $\xi \in \mathscr{Z}$ lying over $\eta: q(\eta)=\xi$. We show that $q$ is biregular in the algebraic sense at $\xi: \mathcal{O}_{\star \times X, \eta} \underset{\rightarrow}{\rightarrow} \mathcal{O}_{x, \xi}$. We denote by $\xi^{\prime}$ the unique point of $Z_{U}$ lying over $\eta^{\prime}=(p, x)$. The morphism $q$ is proper and dominant by Lemma (1.25). Since the question is local we may assume that $q$ is finite and of degree 1 by Lemma (1.25) and by (3.17) Proposition of $[\mathrm{M}] . \quad$ We set $\mathcal{O}_{1}=\mathcal{O}_{\mathscr{\alpha} \times X, \eta}, \mathcal{O}_{2}=\mathcal{O}_{\mathscr{q}, \xi}, \tilde{\mathcal{O}}_{1}=\mathcal{O}_{\mathscr{s} \times X, \eta}^{\mathrm{an}}, \quad \tilde{\mathcal{O}}_{2}=\mathcal{O}_{\mathscr{q}, \xi}^{\mathrm{an}}, \tilde{\mathcal{O}}_{3}=\mathcal{O}_{U \times X, \eta^{\prime}}$, $\tilde{\mathcal{O}}_{4}=\mathcal{O}_{Z, \xi^{\prime}}$ and let $\mathfrak{m}_{i} \subset \mathcal{O}_{i}(i=1,2) \tilde{\mathfrak{m}}_{j} \subset \tilde{\mathcal{O}}_{j}(j=1,2,3,4)$ be the maximal ideals so that we have $\mathfrak{m}_{1} \widetilde{\mathcal{O}}_{1}=\tilde{\mathfrak{m}}_{1}$. We have a commutative diagram 


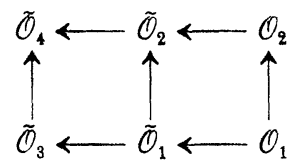

of local morphisms of the local rings. Since of diagram (i) is cartesian and $q^{\prime}$ is isomorphic at $\xi^{\prime}, \tilde{\mathcal{O}}_{1} / \tilde{\mathfrak{m}}_{1} \simeq \tilde{\mathcal{O}}_{3} \tilde{\mathfrak{m}}_{3} \simeq \tilde{\mathcal{O}}_{4} / \tilde{\mathfrak{m}}_{3} \widetilde{\mathcal{O}}_{4} \simeq \tilde{\mathcal{O}}_{2} / \tilde{\mathfrak{m}}_{1} \tilde{\mathcal{O}}_{2} \simeq \widetilde{\mathcal{O}}_{2} / \mathfrak{m}_{1} \tilde{\mathcal{O}}_{1} \tilde{\mathcal{O}}_{2}=$ $\tilde{\mathcal{O}}_{2} / \mathfrak{m}_{1} \tilde{\mathcal{O}}_{2}$ Since $\tilde{\mathcal{O}}_{2}$ is faithfully flat over $\mathcal{O}_{2}$, we have $m_{1} \tilde{\mathcal{O}}_{2} \cap \mathcal{O}_{2}=\mathcal{O}_{1} \tilde{\mathcal{O}}_{2}$. Therefore we get a commutative diagram

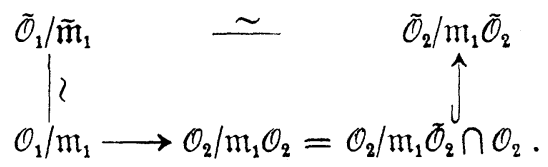

Hence $q$ induces an isomorphism

(ii) $\mathcal{O}_{1} / \mathfrak{m}_{1} \underset{\sim}{\rightarrow} \mathcal{O}_{2} / \mathfrak{m}_{1} \mathcal{O}_{2}$.

We denote by $\varphi$ the morphism $\mathcal{O}_{1} \rightarrow \mathcal{O}_{2}$ induced by $q$. We have an exact sequence of $\mathcal{O}_{1}$-modules

(iii) $0 \rightarrow N \rightarrow \mathcal{O}_{1} \rightarrow \mathcal{O}_{2} \rightarrow M \rightarrow 0$,

where $N=\operatorname{Ker} \varphi, M=\operatorname{Coker} \varphi$. Tensoring the exact sequence with $\mathcal{O}_{1} / \mathfrak{m}_{1} \mathcal{O}_{1}$, we get an exact sequence

$$
\mathcal{O}_{1} / \mathfrak{m}_{1} \mathcal{O}_{1} \rightarrow \mathcal{O}_{2} / \mathfrak{m}_{1} \mathcal{O}_{2} \rightarrow M / \mathfrak{m}_{1} M \rightarrow 0
$$

Hence $M / m_{1} M=0$ by (ii) and consequently $M=0$ by Nakayama's Lemma. Now the exact sequence (iii) becomes

(iv) $0 \rightarrow N \rightarrow \mathcal{O}_{1} \rightarrow \mathcal{O}_{2} \rightarrow 0$.

Tensoring the exact sequence (iv) with the quotient field $K_{1}$ of $\mathcal{O}_{1}$ (the scheme $\mathscr{A}$ is irreducible and reduced), we get

$$
0 \rightarrow N \otimes K_{1} \rightarrow K_{1} \rightarrow \mathcal{O}_{2} \otimes K_{1} \rightarrow 0 \text {. }
$$

Since $q$ is of degree $1, K_{1} \simeq \mathcal{O}_{2} \otimes K_{1}$ and $N \otimes K_{1}=0$. Since $\mathcal{O}_{1}$ is a domain, the submodule $N \subset \mathcal{O}_{1}$ should be 0 and $\mathcal{O}_{1} \widetilde{\mathcal{O}_{2}}$ as required.

Department of Mathematics

Kumamoto University

Kumamoto 860

Japan 